Draft Version September 3, 2018

Preprint typeset using $\mathrm{L}_{\mathrm{A}} \mathrm{EX}$ style emulateapj v. 5/2/11

\title{
ON TESTING THE KERR METRIC OF THE MASSIVE BLACK HOLE IN THE GALACTIC CENTER VIA STELLAR ORBITAL MOTION: FULL GENERAL RELATIVISTIC TREATMENT
}

\author{
FUPENG ZHANG ${ }^{1,2, *}$, YOUJUN LU ${ }^{2, \dagger} \&$ QINGJUAN YU ${ }^{1, \ddagger}$ \\ ${ }^{1}$ Kavli Institute for Astronomy and Astrophysics, Peking University, Beijing, 100871, China; * zhangfupeng@pku.edu.cn; \\ $\ddagger$ yuqj@pku.edu.cn \\ ${ }^{2}$ National Astronomical Observatories, Chinese Academy of Sciences, Beijing, 100012, China; †luyj@nao.cas.cn \\ Draft version September 3, 2018
}

\begin{abstract}
The S-stars, discovered in the vicinity of the massive black hole (MBH) in the Galactic center (GC), are anticipated to provide unique dynamical constraints on the $\mathrm{MBH}$ spin and metric, in addition to the mass. In this paper, we develop a fast full general relativistic method to simultaneously constrain the $\mathrm{MBH}$ mass, spin, and spin direction by considering both the orbital motion of a star close to the $\mathrm{GC} \mathrm{MBH}$ and the propagation of photons from the star to a distant observer. Based on the current observations and dynamical model predictions, we assume six example stars with different semimajor axes $\left(a_{\text {orb }}\right)$ and eccentricities $\left(e_{\text {orb }}\right)$ and numerically calculate their projected trajectories in the sky plane and redshift curves. Two of those stars are set to have orbital configurations similar to that of S0-2/S2 and S0-102. We find that the spin-induced effects on the projected trajectory and redshift curve of a given star, including the leading term by the Lense-Thirring precession and the frame dragging, and the high-order precession due to the quadruple moment, depend on both the absolute value and the direction of the spin. The maximum values of the spin-induced position displacement and the redshift differences of the star over a full orbit may differ by a factor of several to more than one order of magnitude for two cases with significantly different spin directions. The dependence patterns of the position displacements and redshift differences on the spin direction are different, and thus the position and the redshift data are complementary for constraining the MBH spin and its direction. Adopting the Markov Chain Monte Carlo fitting technique, we illustrate that the spin of the GC MBH is likely to be well constrained by using the motion of S0-2/S2 over a period of $\sim 45$ years if the spin is close to one and if the astrometric and spectroscopic precisions can be as high as $\left(\sigma_{\mathrm{p}}, \sigma_{Z}\right) \sim\left(10 \mu \mathrm{as}, 1 \mathrm{~km} \mathrm{~s}^{-1}\right)$, which is expected to be realized by future facilities like the GRAVITY on the Very Large Telescope Interferometer, the thirty meter telescope, and the European extremely large telescope. If $\sigma_{\mathrm{p}}$ and $\sigma_{Z}$ can be further improved by a factor of several, the MBH spin can be well constrained by monitoring S0-2/S2 over a period of $\sim 15$ years. In the mean time, the distance from the Sun to the GC and the MBH mass can also be constrained to an unprecedented accuracy $(0.01 \%-0.1 \%)$. If there exists a star with a semimajor axis that is a few times smaller than, and eccentricity larger, than those of $\mathrm{S} 0-2 / \mathrm{S} 2$, the $\mathrm{MBH}$ spin and its direction can be constrained with high accuracy over a period of $\lesssim 10$ year by future facilities, even if the spin is only moderately large. Our results suggest that long-term monitoring of the motions of stars in the vicinity of the GC $\mathrm{MBH}$ by the next generation facilities is likely to provide a dynamical test, for the first time, to the spin and metric of the GC MBH.
\end{abstract}

Subject headings: Black hole-physics - gravitation - Galaxy: center - Galaxy: nucleus - relativistic processes - stars: kinematics and dynamics

\section{INTRODUCTION}

The Kerr black hole (Kerr 1963) is one of the most simple and elegant solutions to the Einstein field equation of general relativity (GR). It is widely accepted that all astrophysical black holes (BHs), if existing, can be described by the Kerr metric with only two parameters, i.e., the mass and the spin (no-hair theorem). Various lines of evidences for the existence of $\mathrm{BHs}$ have been accumulated in the past several decades, mainly based on dynamical measurements of the masses of these objects (e.g., Magorrian et al. 1998; Kormendy \& Ho 2013; Naravan \& McClintock 2013). One of the strongest evidences is provided by the long-term monitoring of the motions of stars in the Galactic center (GC), which suggests, almost exclusively, the existence of a massive black hole (MBH; with mass $\left.\sim 4 \times 10^{6} M_{\odot}\right)$ in the GC (see Ghez et al. 2008; Gillessen et al. 2009; Mever et al. 2012). However, it is still not clear whether these objects can be fully described by the Kerr metric or not.

A crucial step to check whether an astrophysical BH is described by the Kerr metric is to accurately constrain/measure the BH spin through spin-induced GR effects. Recently, some progress has been made in measuring the spins of both stellar-mass BHs in the Milky Way (i.e., McClintock et al.|2011, and references therein) and MBHs in the centers of active galactic nuclei (e.g., Reynolds 2013, and references therein). These measurements are obtained by modeling the intrinsic X-ray continuum and its reflection components (e.g., the $\mathrm{Fe}$ $\mathrm{K} \alpha$ line and the reflection continuum) from each $\mathrm{BH}-$ accretion disk system, which may suffer from various uncertainties in accretion disk models. Therefore, independent determinations of the spins of $\mathrm{BHs}$ are of fundamen- 
tal importance for testing the no-hair theorem, GR, and a deep understanding of the space and time.

The MBH system in the GC, massive and in close proximity to us, provides a unique laboratory for testing the no-hair theorem and the GR due to the following reasons. First, the angular size of the expected shadow/image of the $\mathrm{GC} \mathrm{MBH}$ is the largest one among those of MBHs, which makes the $\mathrm{GC} \mathrm{MBH}$ the best target for the event horizon telescope (e.g., Doeleman et al. 2009). Second, the $\mathrm{GC} \mathrm{MBH}$ is the only $\mathrm{MBH}$ which has stars in its vicinity that can be detected individually. Long-term monitoring of the motions of those stars in the immediate vicinity of the $\mathrm{MBH}$ offers a novel opportunity to probe various GR effects, including the periastron advancement, the Lense-Thirring precession, and the frame dragging (see Jaroszvnski 1998; Fragile \& Mathews 2000; Rubilar \& Eckart 2001; Weinberg et al. 2005; Preto \& Saha 2009; Angélil et al. 2010; Angélil \& Saha 2010; Merritt et al. 2010; Angélil \& Saha 2011; Iorio 2011a,b). Currently, the detected S-stars rotating around the $\mathrm{GC} \mathrm{MBH}$ that have the smallest semimajor axes are $\mathrm{S} 0-2 / \mathrm{S} 2$ and $\mathrm{S} 0-102$, which have semimajor axes of $980 \mathrm{AU}$ and $850 \mathrm{AU}$ and pericenter distances of $120 \mathrm{AU}$ and $270 \mathrm{AU}$, respectively (Schödel et al. 2012; Ghez et al. 2008; Gillessen et al. 2009; Mever et al. 2012). It is also anticipated that there are some stars (fainter than the S-stars) and pulsars hiding within the orbit of S0-2/S2 and S0-102 (see Zhang et al. 2013, 2014, and references therein). The GR effects, e.g., the LenseThirring precession and the frame dragging, which are inherited from the $\mathrm{MBH}$ spin, should hide in the apparent trajectories of these stars (e.g., Jaroszynski 1998) and in the redshift curve of the star (Angélil et al. 2010), however, they are almost unreachable by current facilities.

A number of next-generation facilities are currently in progress, such as the GRAVITY on the Very Large Telescope Interferometer (VLTI), the thirty meter telescope (TMT), and the European extremely large telescope (EELT). With these facilities, not only will there be great advancement in the precisions of the astrometric and spectroscopic measurements, but also some stars, fainter and closer to the central MBH than the known S-stars, may be revealed (e.g., Zhang et al. 2013). It is promising to dynamically constrain the $\mathrm{MBH}$ spin and metric by using the relativistic orbital motions of those stars.

Various spin-induced GR effects have been investigated by many authors (e.g., Jaroszynski 1998; Rubilar \& Eckart 2001; Angélil et al. 2010; Angélil \& Saha 2010; Merritt et al. 2010; Angélil \& Saha 2011; Iorio 2011a.b), however, most of those studies are based on perturbative approximations rather than full general relativistic calculations and do not consider how tight the constraint on the $\mathrm{MBH}$ spin could be obtained. In this paper, we develop a full general relativistic method to investigate the spin-induced GR effect by considering both the relativistic orbital motion of a star in the vicinity of the $\mathrm{GC} \mathrm{MBH}$ and the propagation of photons from the star to a distant observer. Using this method and the Markov Chain Monte Carlo (MCMC) fitting scheme, we investigate how a constraint on the $\mathrm{MBH}$ spin and how accurate the constraint can be obtained by using the orbital motion of an example star, e.g., S0-2/S2, for a given set of astrometric and spectroscopic accuracies of an instrument or a telescope.

This paper is organized as follows. By adopting the Kerr metric to describe the curved spacetime around the $\mathrm{MBH}$, we introduce the motion equations for a star rotating around the $\mathrm{MBH}$ and for photons propagating in the spacetime in Section 2 . We introduce the numerical method to solve the GR motion equations for the star and the ray-tracing technique to trace back those photons propagating from the star to a distant observer in Sections 3 and 4 . In Section 5, we summarize the main results of our calculations on the orbital motions of some example stars and the propagation of photons from those stars to the distant observer. Two of those example stars have similar orbital configurations as those of S02/S2 and S0-102; and the others are set according to the predictions on the probability distributions of the semimajor axis and eccentricity of a star, which is expected to be the closest one to the GC MBH as predicted by some dynamical models. In Section 6] we describe the procedures of an MCMC fitting to the mock observations of the example stars, assuming astrometric and spectroscopic accuracies of the future observations. By doing this, we further investigate how accurate the constraint on the MBH spin and metric can be obtained by using the relativistic orbital motions of a star, e.g., S0-2/S2, with the next generation facilities. Discussions on some related issues are given in Section 7 and conclusions are summarized in Section 8

\section{KERR METRIC AND MOTION EQUATIONS}

The curved spacetime around an astrophysical BH may be described by the Kerr metric (Kerr 1963), which can be expressed in the Boyer-Lindquist coordinates $(t, r, \theta, \phi)$ as

$$
d s^{2}=-e^{2 \nu} d t^{2}+e^{2 \psi}(d \phi-\omega d t)^{2}+e^{2 \mu_{1}} d r^{2}+e^{2 \mu_{2}} d \theta^{2},
$$

where

$$
\left\{\begin{array}{l}
e^{2 \nu}=\Sigma \Delta / A \\
e^{2 \psi}=A \sin ^{2} \theta / \Sigma \\
e^{2 \mu_{1}}=\Sigma / \Delta \\
e^{2 \mu_{2}}=\Sigma \\
\omega=2 a r / A \\
\Sigma=r^{2}+a^{2} \cos ^{2} \theta \\
\Delta=r^{2}-2 r+a^{2} \\
A=\left(r^{2}+a^{2}\right)^{2}-a^{2} \Delta \sin ^{2} \theta
\end{array}\right.
$$

and $a$ is the dimensionless spin parameter of the $\mathrm{MBH}$ (Boyer \& Lindquist 1967). For simplicity, we set $G=$ $c=M_{\bullet}=r_{g}=G M_{\bullet} / c^{2}=1$ above, $G, c, M_{\bullet}$ and $r_{g}$ are the gravitational constant, the speed of light, the $\mathrm{MBH}$ mass, and the gravitational radius, respectively.

In the Kerr metric, the motion of a particle, a star, or a photon, is controlled by the following equations (Bardeen et al. 1972; Misner et al. 1973; Chandrasekhar 1983)

$$
\begin{aligned}
\Sigma \dot{r} & = \pm \sqrt{R} \\
\Sigma \dot{\theta} & = \pm \sqrt{\Theta} \\
\Sigma \dot{\phi} & =-a+\lambda / \sin ^{2} \theta+a T / \Delta, \\
\Sigma \dot{t} & =-a^{2} \sin ^{2} \theta+a \lambda+\left(r^{2}+a^{2}\right) T / \Delta,
\end{aligned}
$$

where overdot represents an ordinary derivative with respect to an affine parameter $\tau$, and $T=r^{2}+a^{2}-\lambda a$. The 
quantities $R$ and $\Theta$ in Equations (31) and (4) are given by

$$
\begin{aligned}
R= & \left(1-\xi^{2}\right) r^{4}+2 \xi^{2} r^{3}+\left[a^{2}\left(1-\xi^{2}\right)-q^{2}-\lambda^{2}\right] r^{2} \\
& +2\left[(a-\lambda)^{2}+q^{2}\right] r-a^{2} q^{2}
\end{aligned}
$$

and

$$
\Theta=q^{2}-\left[a^{2}\left(\xi^{2}-1\right)+\lambda^{2} / \sin ^{2} \theta\right] \cos ^{2} \theta,
$$

respectively, where $\xi=m / E$ ( $\xi=0$ for photons), $\lambda=$ $L_{z} / E$, and $q^{2}=Q / E^{2}$. The constants of motion are the rest mass $m$, the energy at infinity $E$, the azimuthal angular momentum $L_{z}$, and the Carter's constant $Q$.

For a star in the immediate vicinity of an $\mathrm{MBH}$ that is monitored by a distant observer, the GR effects encoded in the measurements can be divided into two parts. First, the orbital motion of the star rotating around the $\mathrm{MBH}$ is affected by the GR effects, such as the periastron advancement, the Lense-Thirring precession, and the frame dragging. Second, the propagation of photons from the star to the distant observer is also affected by the GR effects, including the gravitational redshift for those photons to climb out of the $\mathrm{MBH}$ potential, and the gravitational bending of the photon trajectories if the distance of those photons to the central MBH is small. Therefore, it is necessary to consider both the relativistic motion of the star and the propagation of photons from the star to the distance observer, simultaneously. Below, we introduce the full general relativistic numerical methods to calculate the relativistic orbits of stars and the trajectories of photons in the Kerr metric, respectively.

\section{RELATIVISTIC ORBITS OF STARS}

The orbital motion of a star in the vicinity of the central $\mathrm{MBH}$, with a fixed mass $\left(M_{\bullet}\right)$, spin $(a)$ and its direction 1 is determined by equations (3)-(6) once the initial conditions are known. The initial conditions of the star can be given by its position and tetrad velocity at a time $t_{\star, 0}$ in the Boyer-Lindquist coordinates, i.e., $\mathbf{r}_{\star, 0}=\left(t_{\star, 0}, r_{\star, 0}, \theta_{\star, 0}, \phi_{\star, 0}\right)$ and $\mathbf{u}_{\star, 0}=$ $\left(u_{\star, 0}^{t}, u_{\star, 0}^{r}, u_{\star, 0}^{\theta}, u_{\star, 0}^{\phi}\right)$. Hereafter, $t_{\star, 0}$ is set to be zero for simplicity, if not otherwise stated. At any given time $t_{\star}$, the three-velocity $\mathbf{v}_{\star}=\left(v_{\star}^{r}, v_{\star}^{\theta}, v_{\star}^{\phi}\right)$ in the space of the local non-rotating rest frame (LNRF) associated with $\left(r_{\star}, \theta_{\star}, \phi_{\star}\right)$ can be transformed from the tetrad velocity $\mathbf{u}_{\star}=\left(u_{\star}^{t}, u_{\star}^{r}, u_{\star}^{\theta}, u_{\star}^{\phi}\right)=(\dot{t}, \dot{r}, \dot{\theta}, \dot{\phi})$ as (see Bardeen et al. 1972; ; Bardeen 1973; Misner et al. 1973)

$$
\begin{gathered}
v_{\star}^{r}=u_{\star}^{r} e^{\mu_{1}-\nu} / u_{\star}^{t}, \\
v_{\star}^{\theta}=u_{\star}^{\theta} e^{\mu_{2}-\nu} / u_{\star}^{t}, \\
v_{\star}^{\phi}=\left(u_{\star}^{\phi} / u_{\star}^{t}-\omega\right) e^{\psi-\nu} .
\end{gathered}
$$

The tetrad velocity $\mathbf{u}_{\star}$ can be reversely transformed from the three-velocity $\mathbf{v}_{\star}$ in the LNRF frame.

If the position and the three-velocity of a star in the LNRF frame are provided, the values of $\xi$ and $\lambda$ can be derived as

$$
\xi=1 /\left[\gamma\left(e^{\nu}+\omega e^{\psi} v_{\star}^{\phi}\right)\right]
$$

1 The MBH mass, spin, and its direction are assumed to be fixed values because the time duration of the orbital motion considered in this study is short and no more than several decades, and thus there should be no significant evolution in the MBH mass and spin vector. and

$$
\lambda=A v_{\star}^{\phi} /\left(2 a r v_{\star}^{\phi}+\Gamma\right),
$$

respectively, where

$$
\gamma=\left(1-\overrightarrow{\mathbf{v}}_{\star} \cdot \overrightarrow{\mathbf{v}}_{\star}\right)^{-1 / 2},
$$

and

$$
\Gamma=\left[A\left(\Delta-a^{2} \sin ^{2} \theta\right)+4 a^{2} r^{2} \sin ^{2} \theta\right] / \Sigma \sqrt{\Delta} \sin \theta .
$$

Substituting $\xi$ and $\lambda$ into Equations (8) and (10), we have

$$
q^{2}=\Sigma\left(\gamma v_{\star}^{\theta}\right)^{2}+\cos ^{2} \theta\left[a^{2}\left(\xi^{2}-1\right)+\lambda^{2} / \sin ^{2} \theta\right] .
$$

We define two pseudo-Cartesian coordinate systems for the convenience of relating the motion of a star in the vicinity of the $\mathrm{MBH}$ with the Boyer-Lindquist coordinates $(r, \theta, \phi)$ to that seen by a distant observer. The first pseudo-Cartesian coordinate system $x^{\prime} y^{\prime} z^{\prime}$ is defined in the rest frame of the distant observer (denoted as the observer's frame hereafter). The direction of the $z^{\prime}$-axis is pointing from the MBH to the distant observer, and the $x^{\prime} y^{\prime}$ plane represents the sky plane of the observer. The second pseudo-Cartesian coordinate system $x y z$ is defined to relate the Boyer-Lindquist coordinate $(r, \theta, \phi)$ to an orthogonal coordinate system $(x, y, z)$ with $\vec{z}$ representing the direction of the spin vector (see a similar definition in Angélil et al. 2010). Because the star is usually far away from the event horizon of the central MBH, the coordinates of the star in the $x y z$ frame may be approximated by

$$
\left\{\begin{array}{l}
x=r \sin \theta \cos \phi \\
y=r \sin \theta \sin \phi, \\
z=r \cos \theta .
\end{array}\right.
$$

In this $x y z$ frame, the direction of the $z$-axis represents the spin direction of the $\mathrm{MBH}$, the $y$-axis represents the intersection line of the equatorial plane of the $\mathrm{MBH}$ with the sky plane of the distant observer. The LNRF frame is rotating with an angular velocity of $\omega$ relative to the distant observer's frame. However, $\omega$ is relatively small since the star is usually far away from the event horizon of the MBH. Therefore, the rotation of the LNRF frame with respect to the distant observer's frame can be neglected when relating the initial conditions to the Newtonian approximations.

Figure 1] shows the configuration of the stellar-MBH system and the two pseudo-Cartesian coordinate systems defined above. As seen from Figure 1, the spin direction $\vec{z}$ is determined by two angles in the observer's frame $x^{\prime} y^{\prime} z^{\prime}$, i.e., the angle $(i)$ between $\vec{z}$ and $\vec{z}^{\prime}$, and the angle $(\epsilon)$ between the projection of $\vec{z}$ on the $x^{\prime} y^{\prime}$ plane and $-\vec{x}^{\prime}$. The angle between the $y$ axis and the $y^{\prime}$ axis is the same as $\epsilon$. Therefore, a vector defined in the observer's frame can be approximately transformed to the LNRF frame (or the MBH's frame) by first rotating the $x^{\prime} y^{\prime} z^{\prime}$ frame around the $z^{\prime}$ axis counter-clockwise by an angle of $\epsilon$ and then rotating it around the new $y^{\prime}$ axis clockwise by an angle of $i$. For example, a vector $\mathbf{n}^{\prime}$ in the observer's frame can be transformed to that in the LNRF frame $\mathbf{n}$ by $\mathbf{n}=\mathbf{M n}^{\prime}$, where $\mathbf{M}$ is a rotation matrix given by

$$
\mathbf{M}=\left(\begin{array}{ccc}
\cos i \cos \epsilon & \cos i \sin \epsilon & \sin i \\
-\sin \epsilon & \cos \epsilon & 0 \\
-\sin i \cos \epsilon & -\sin i \sin \epsilon & \cos i
\end{array}\right) .
$$



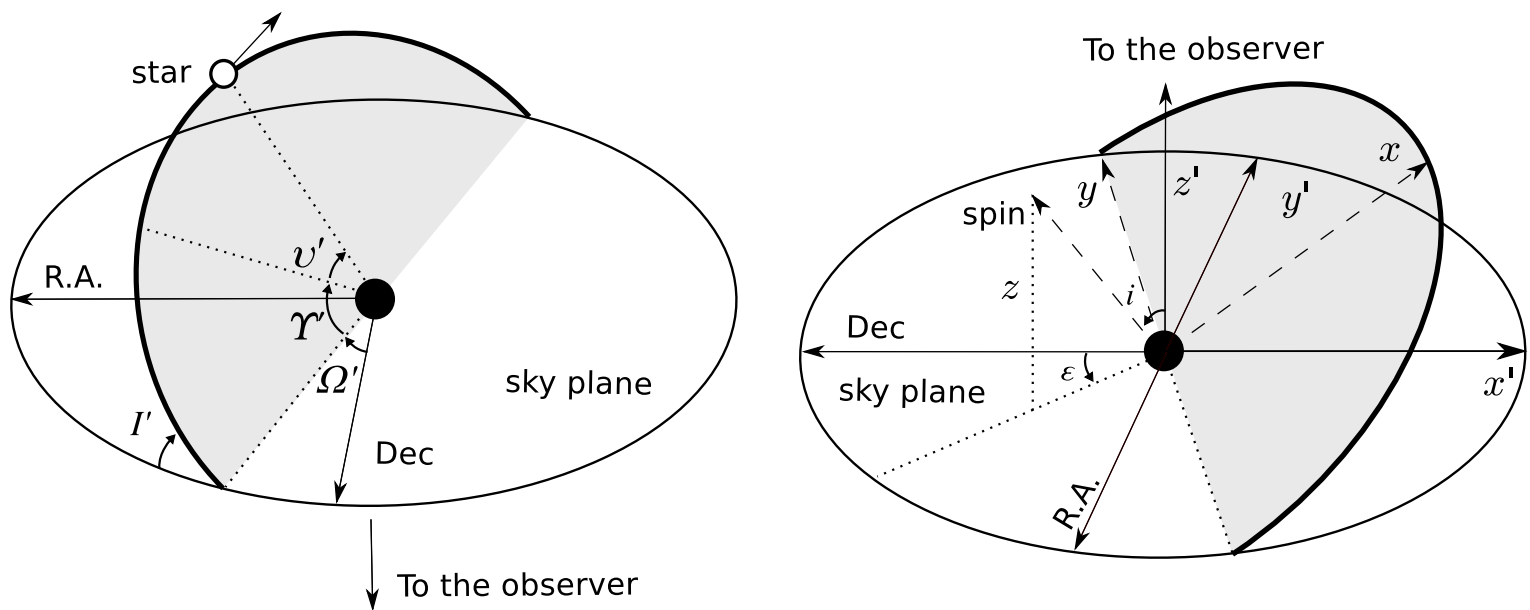

FIG. 1. - Schematic diagram for the star-MBH system and the coordinate systems. Right panel: a pseudo-Cartesian coordinate $\left(x^{\prime}, y^{\prime}, z^{\prime}\right)$ is defined in the rest frame of a distant observer (i.e., the observer's frame), where the $x^{\prime} y^{\prime}$ plane represents the sky plane of the observer and is taken as the reference plane, the $z^{\prime}$ axis represents the line of sight, and the $x^{\prime}$ axis is taken as the reference direction on the sky plane. Another pseudo-Cartesian coordinate $x y z$ is defined to relate the Boyer-Lindquist coordinates $(r, \theta, \phi)$ to orthogonal coordinates $(x, y, z)$, where the $z$ axis represents the spin direction of the MBH and the $y$ axis represents the intersection line of the MBH equatorial plane with the observer's sky plane. The direction of the MBH spin in the observer's frame is therefore described by two angles, i.e., the angle the $z$ axis and the $z^{\prime}$ axis $(i)$ and the angle between the projection vector of the $x$ axis on the $x^{\prime} y^{\prime}$ plane and the $x^{\prime}$ axis $(\epsilon)$. The directions of the R.A. $\left(-\vec{y}^{\prime}\right)$ and Dec. $\left(-\vec{x}^{\prime}\right)$ are marked in this figure. Left panel: the orbit of the star may be approximated as a Newtonian ellipse in the observer's frame, especially when the semimajor axis of the star is large, which is described by six orbital elements, i.e., the semimajor axis $a_{\text {orb }}$, eccentricity $e$, the longitude of ascending node $\Omega^{\prime}$, argument of periapsis $\Upsilon^{\prime}$, true anomaly $v^{\prime}$ and inclination $I^{\prime}$, respectively.

In the Newtonian case, the orbital motion of a star rotating around a massive object is determined by six orbital elements, i.e., the semimajor axis $a_{\text {orb }}$, the eccentricity $e_{\text {orb }}$, the longitude of the ascending node $\Omega^{\prime}$, the argument of the pericenter $\Upsilon^{\prime}$, the true anomaly $v^{\prime}$, and the orbital inclination angle $I^{\prime}$ (see Figure 11). For convenience in comparison with the Newtonian orbits currently determined for a few GC S-stars with the smallest semimajor axis, in our following simulations of the orbital motions of some (example) stars, we set their initial conditions by fixing the six orbital elements at a given moment in the distant observer's frame 2 Then, the values for the four-position $\mathbf{r}_{\star, 0}$ and the tetrad-velocity $\mathbf{u}_{\star, 0}$ of a star can be approximately obtained by the following procedures.

1. The initial position $\left(x_{\star, 0}^{\prime}, y_{\star, 0}^{\prime}, z_{\star, 0}^{\prime}\right)$ and the three velocity $\left(v_{\star, 0}^{\prime x}, v_{\star, 0}^{\prime y}, v_{\star, 0}^{\prime z}\right)$ of the star in the distant observer's frame are first obtained from the six orbital elements initially set.

2. Transforming the position and the velocity of the star in the distant observer's frame into those in the LNRF frame, i.e., $\vec{r}_{\star, 0}=\left(x_{\star, 0}, y_{\star, 0}, z_{\star, 0}\right)$ and $\vec{v}_{\star, 0}=\left(v_{\star 0}^{x}, v_{\star, 0}^{y}, v_{\star, 0}^{z}\right)$, by the rotation given in Equation (18), and then transforming these two vectors from that using the $x y z$ coordinates into that using the $r \theta \phi$ coordinates.

3. Transforming $\vec{r}_{\star, 0}$ and $\vec{v}_{\star, 0}$ to the four-position $\mathbf{r}_{\star, 0}$ and the tetrad-velocity $\mathbf{u}_{\star, 0}$ in the Boyer-Lindquist coordinates according to Equations (9)-(11).

2 The orbital elements set here are used in a simple way to generate the initial conditions of a star moving in the Boyer-Lindquist coordinates. The elliptic orbit defined by these orbital elements is only taken as a Newtonian approximation to the real orbit of a star in the Boyer-Lindquist coordinates. These orbital elements may lose their original meaning in the curved spacetime around a Kerr MBH.
Once the initial conditions are given for a star, the motion parameters $\lambda, \xi$, and $q$ can be obtained by Equations (12), (13), and (16), respectively. With those constants of motion, the orbital motion of a star, in principle, can be obtained by integrating the motion Equations (3) - (6) . However, it is required to frequently judge the sign " \pm " at the left side of the motion Equations (3) and (4) in the numerical integrations. To avoid this complication, one may use the following equations to replace the motion Equations (3) and (44).

$$
\begin{gathered}
\Sigma \dot{r}=\Delta p_{r} \\
\Sigma \dot{\theta}=p_{\theta} \\
\Sigma \dot{p}_{r}=\frac{1}{2 \Delta} \frac{\partial R}{\partial r}-R \frac{\partial \Theta}{\partial \theta} \\
\Sigma \dot{p}_{\theta}=\frac{1}{2} \frac{\partial \Theta}{\partial \theta}
\end{gathered}
$$

We use the code DORPI5 based on the explicit fifth (fourth)-order Runge Kutta method (Dormand \& Prince 1980; Hairer et al. 1993) to integrate the motion Equations (19)-(22) and (5) and (6) to obtain the orbit motion of a star. We set the relative integration errors to be $\leq 10^{-12}$ for all the position and momentum quantities $\left(r, \theta, \phi, p_{r}, p_{\theta}, p_{\phi}\right)$ in those equations, which are sufficient for the convergence of the numerical results and the required accuracy in this study.

\subsection{Orbits of Example Stars}

Observations in the past two decades have revealed a number of GC S-stars rotating around the MBH with semimajor axes in the range of $\sim 4000-800$ AU. Among them, the two with the smallest semimajor axes are S02/S2 and S0-102 (Ghez et al. 2008; Gillessen et al. 2009; Mever et al. 2012). These stars have been very useful in determining the MBH mass, and it is expected that S0-2/S2 (or a star within the orbit of S0-2/S2) will be 

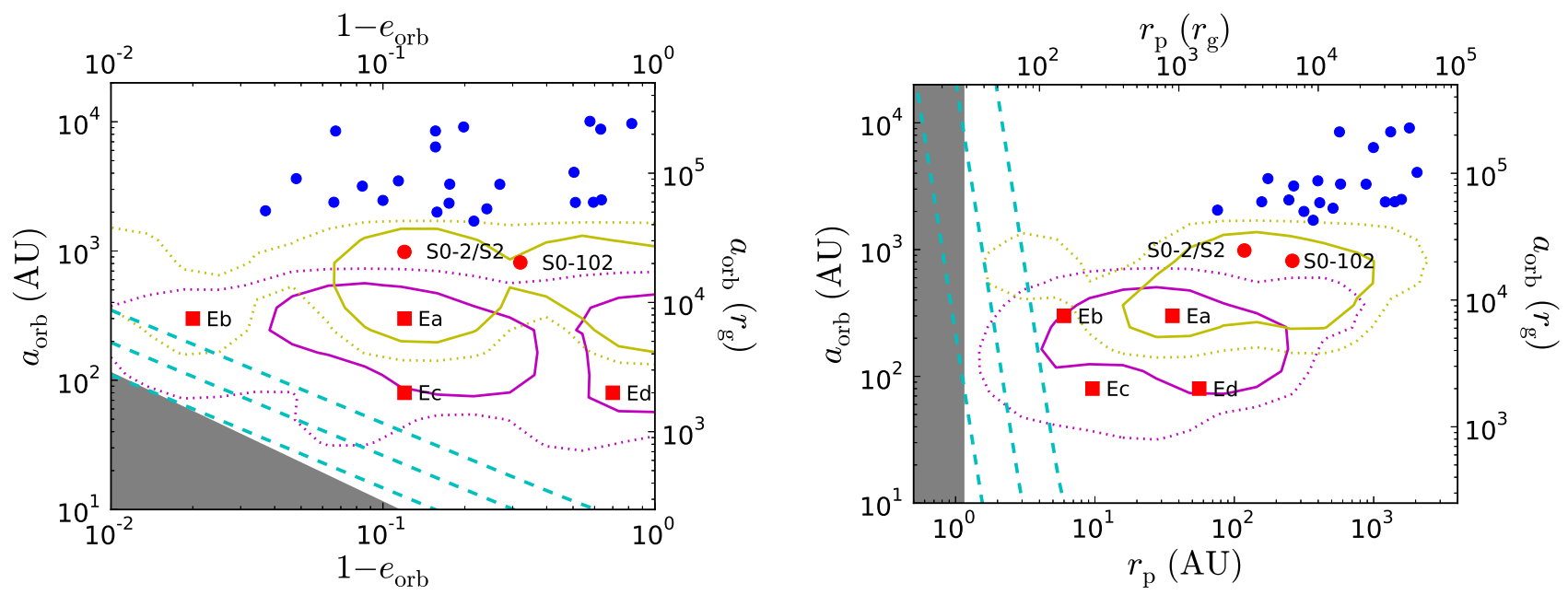

FIG. 2.- Orbital distribution of some detected GC S-stars and the possibly existing star that is closest to the GC MBH in the semimajor axis versus eccentricity plane (left panel) and in the semimajor axis versus the distance to the pericenter plane (right panel). The tick labels at the right side of each panel and the top side of the right panel are in units of gravitational radius $r_{\mathrm{g}}=G M_{\bullet} / c^{2}$, while the tick labels at the left side of each panel and the bottom side of the right panel are measured with the astronomical unit AU. The solid circles represent those currently detected GC S-stars within a distance of $4000 \mathrm{AU}$ from the GC MBH. S0-2/S2 and S0-102 are the two with the smallest semimajor axes among the GC S-stars (see Meyer et al. 2012). The probabilities of a predicted star with mass in the range $1-7 M_{\odot}$ that is closest to the MBH within the yellow and the magenta solid (dotted) curves are $85 \%(98 \%$ ) and $66 \%$ (98\%), resulting from the model "Disk-IM2" and "Disk-IM0" in Zhang et al. (2013), respectively, by assuming that the GC S-stars are the captured components of stellar binaries injected into the vicinity of the $\mathrm{MBH}$. The cyan dashed lines give the gravitational radiation timescale of a star with mass $3 M_{\odot}$ rotating around the $\mathrm{MBH}: 10^{8}, 10^{7}$, and $10^{6}$ years from top to bottom in the left panel (or from right to left in the right panel). The pericenter distances of the stars, if existing, in the shaded region are smaller than the tidal disruption radii for a star with mass $3 M_{\odot}$, and thus stars almost cannot exist in this region.

an important dynamical probe to the GR effects of the $\mathrm{GC} \mathrm{MBH}$. It is also anticipated that there are some stars fainter than the GC S-stars existing within the orbits of S0-2/S2 and S0-102, which could be even better probes for the GR effects. Zhang et al. (2013) predicted the probability density distribution in the semimajor axis versus eccentricity plane of such stars (with mass in the range of $\left.1-7 M_{\odot}\right)$, by assuming that these stars and the GC S-stars are the captured components of stellar binaries that are tidally broken up in the vicinity of the $\mathrm{GC}$ $\mathrm{MBH}$.

Figure 2 shows both the orbital distribution of the detected GC S-stars (solid circles) and the probability density distribution of the expected innermost star 3 (color contours) in the semimajor axis versus eccentricity plane (panel (a)) and the semimajor axis versus pericenter distance plane (panel (b)). The magenta (yellow) solid and dotted contours represent a probability for the expected innermost star with mass in the range of $1-7 M_{\odot}$ of $85 \%$ and $98 \%$ (or $66 \%$ and $98 \%$ ), resulted from the "Disk-IM0" (or "Disk-IM2") model in Zhang et al. (2013), respectively. The semimajor axis and eccentricity of the expected innermost star are in the range of $\sim 100-1000 \mathrm{AU}$ and $0-0.99$, respectively. The dotted lines in Figure 2 give the gravitational radiation timescale of a star with mass of $3 M_{\odot}$ rotating around the $\mathrm{MBH}$ with mass $4 \times 10^{6} M_{\odot}: 10^{8}$, $10^{7}, 10^{6}$ years from top to bottom (Equation (5.6) in Peters 1964 or Equation (39) in Yu \& Tremaine 2003). A star should be tidally disrupted if it approaches the $\mathrm{MBH}$ within a distance of $r_{\mathrm{tid}}=\left(\eta^{2} M_{\bullet} / m_{\star}\right)^{1 / 3} r_{\star} \simeq$

3 Here the "innermost" star means the star with the smallest semimajor axis. $\left(\eta^{2} M_{\bullet} / m_{\star}\right)^{1 / 3}\left(m_{\star} / M_{\odot}\right)^{0.47} R_{\odot}$, where $m_{\star}$ is the mass of the star and $\eta$ is set to be 2.21 for a homogeneous, incompressible body (see Magorrian \& Tremaine 1999). The upper (right) boundary of the shaded region in the left (right) panel of Figure 2 represents the tidal radius of stars $\left(r_{\text {tid }}\right)$ with mass $3 M_{\odot}$ rotating around the $\mathrm{MBH}$. In principle, stars with masses $\geq 3 M_{\odot}$ cannot exist in the shaded region. The probability of existing stars in the region between the top (right) dashed line and the upper (or right) boundary of the shaded region in the left (or right) panel is substantially suppressed because of the rapid orbital decay of a star in this region due to gravitational wave radiation.

According to the current observations and theoretical expectations, we select six example stars as listed in Table 11. Two of the example stars have similar orbital properties as those of S0-2/S2 and S0-102, respectively, which have the smallest semimajor axes among the detected GC S-stars (see Ghez et al. 2008; Gillessen et al. 2009; Mever et al. 2012). The other four example stars, i.e., Ea, Eb, Ec, and Ed (see Table 1), are set according to the probability distribution of the expected innermost star shown in Figure 2. The semimajor axes, eccentricities, and pericenter distances of the example stars Ea, Eb, Ec, and Ed are (300 AU, 0.88, $36 \mathrm{AU})$, (300 AU, 0.98, $6 \mathrm{AU}),(80 \mathrm{AU}, 0.88,9.6 \mathrm{AU})$, and (80 AU, 0.3, $56 \mathrm{AU})$, respectively. The probability for the existence of a star like the example star Ea is probably high. There are some chances for the existence of a star like the example stars Eb, Ec, or Ed in the vicinity of the $\mathrm{MBH}$, though the chances are not very high. Note that the semimajor axis and eccentricity listed there are only taken as the Newtonian approximation to the orbit of each example star, which are useful for setting the initial conditions. 
TABLE 1

ORBITAL PARAMETERS FOR EXAMPLE STARS

\begin{tabular}{|c|c|c|c|c|c|c|c|c|c|c|c|}
\hline \multirow{2}{*}{ Name } & \multicolumn{3}{|c|}{$a_{\text {orb }}$} & \multirow{2}{*}{$e_{\mathrm{orb}}$} & \multirow{2}{*}{ I } & \multirow{2}{*}{$\Omega^{\prime}$} & \multirow{2}{*}{$\Upsilon^{\prime}$} & \multirow{2}{*}{$T_{0}^{d}$} & \multirow{2}{*}{$a$} & \multirow{2}{*}{$i^{e}$} & \multirow{2}{*}{$\epsilon^{e}$} \\
\hline & $\mathrm{AU}^{a}$ & $r_{\mathrm{g}}{ }^{b}$ & $\operatorname{mas}^{c}$ & & & & & & & & \\
\hline $\mathrm{S} 0-2 / \mathrm{S} 2$ & 984 & 24949 & 123 & 0.88 & $135^{\circ}$ & $225^{\circ}$ & $63^{\circ}$ & 2.32 & 0.99 & $45^{\circ}$ & $200^{\circ}$ \\
\hline S0-102 & 848 & 21500 & 106 & 0.68 & $151^{\circ}$ & $175^{\circ}$ & $185^{\circ}$ & 9.5 & 0.99 & $45^{\circ}$ & $180^{\circ}$ \\
\hline $\mathrm{Ea}$ & 300 & 7606 & 37.5 & 0.88 & $45^{\circ}$ & $0^{\circ}$ & $0^{\circ}$ & 0 & 0.99 & $45^{\circ}$ & $180^{\circ}$ \\
\hline $\mathrm{Eb}$ & 300 & 7606 & 37.5 & 0.98 & $45^{\circ}$ & $0^{\circ}$ & $0^{\circ}$ & 0 & 0.99 & $45^{\circ}$ & $180^{\circ}$ \\
\hline Ec & 80 & 2028 & 10 & 0.88 & $45^{\circ}$ & $0^{\circ}$ & $0^{\circ}$ & 0 & 0.99 & $45^{\circ}$ & $180^{\circ}$ \\
\hline $\mathrm{Ed}$ & 80 & 2028 & 10 & 0.30 & $45^{\circ}$ & $0^{\circ}$ & $0^{\circ}$ & 0 & 0.99 & $45^{\circ}$ & $180^{\circ}$ \\
\hline
\end{tabular}

Note. - ${ }^{a}$ In units of AU. ${ }^{b}$ In units of the gravitational radius $r_{\mathrm{g}}=G M_{\bullet} / c^{2}$ and the MBH mass $M_{\bullet}$ is assumed to be $4 \times 10^{6} M_{\odot} .{ }^{c}$ In units of mas by assuming the distance from the $\mathrm{MBH}$ to sun $R_{\mathrm{GC}}=8 \mathrm{kpc}$. $d$ Time of pericentric passage, with respect to the year 2000 in order to obtain mock observations for S0-2/S2, S0-102, and other possibly existing stars by future facilities. $e$ The MBH spin direction is set for the case for S0-2/S2 in order to have an intermediate spin-induced effect; and it is set for the cases of other stars arbitrary to the values listed in the table.

\section{PHOTON PROPAGATION: RAY-TRACING}

We consider a photon propagating from a star in the vicinity of an $\mathrm{MBH}$ to a distant observer in this section. The position of the star at each moment $\left(t_{\star}, r_{\star}, \theta_{\star}, \phi_{\star}\right)$ is set by its orbital motion as obtained above in Section 3 once the initial conditions are fixed. The position of the distant observer is fixed at $\left(r_{\mathrm{o}}, \theta_{\mathrm{o}}, \phi_{\mathrm{o}}\right)=\left(R_{\mathrm{GC}}, i, 0^{\circ}\right)$, where $R_{\mathrm{GC}}$ is the distance from the Sun to the GC and set to be $8 \mathrm{kpc}$ if not otherwise stated. The photon trajectory may be bended due to the curved spacetime of the $\mathrm{MBH}$, and the energy of the photon when it is received by the distant observer is different from its original value emitting from the star due to the gravitational redshift and the Doppler shift. To account for those effects, we use the ray-tracing technique to trace back photons from the distant observer to the star in the Boyer-Lindquist coordinate system. The ray-tracing technique is detailed in Appendix $\mathrm{A}$, where both Jacobian elliptic functions (Bvrd \& Friedman 1954; Abramowitz \& Stegun 1972) and the Gauss-Kronrod integration scheme (e.g., see Rauch \& Blandford 1994) are adopted to integrate the motion equations.

The apparent position of the star in the distant observer's sky plane at a given time $t_{\mathrm{o}}$ is described by two impact parameters, $(\alpha, \beta)$, which map the position of the $\operatorname{star}\left(t_{\star}, r_{\star}, \theta_{\star}, \phi_{\star}\right)$ and are measured relative to the center of the $\mathrm{MBH}$. The impact parameter $\alpha$ is the apparent displacement of the image perpendicular to the projected axis of symmetry of the $\mathrm{MBH}$, and $\beta$ is the apparent displacement parallel to the projected axis of symmetry in the sense of the angular momentum of the $\mathrm{MBH}$. For photons, $\xi=0$, the values of the other two constants of motion, $\lambda$ and $q^{2}$ in Equations (7) and (8), can be determined by $\alpha$ and $\beta$ as

$$
\begin{gathered}
\lambda=-\alpha \sin i, \\
q^{2}=\beta^{2}+\left(\alpha^{2}-a^{2}\right) \cos ^{2} i .
\end{gathered}
$$

For details, see Cunningham \& Bardeen (1973), Chandrasekhar (1983), and Karas et al. (1992). The star may have more than one image because of the gravitational lensing effect. For the majority of the cases investigated in this study, however, only one image is dominant because the star is normally far away from the Einstein radius of the central $\mathrm{MBH}$ and the lensing effect is not significant. We may trace a photon with given impact parameters $(\alpha, \beta)$ from the distant observer to the vicinity of the $\mathrm{MBH}$ by solving Equations (3)- (6), and check whether the photon can reach the surface of the star at a given moment.

According to the motion Equations (3) and (4), we have

$$
\int^{r} \frac{d r}{\sqrt{R}}=\int^{\theta} \frac{d \theta}{\sqrt{\Theta}} .
$$

For a photon propagating from the star to the distant observer, the $\theta$-integral at the right side of the above equation can be obtained by integrating over $\theta$ from $\theta_{\mathrm{o}}$ to $\theta_{\star}$ (for details, see Appendix A.1. When $\theta=\theta_{\star}$, the radial position of the photon is denoted as $r_{\text {hit }}$. Therefore, the $r$-integral at the left side can be obtained by integrating over $r$ from $r_{\mathrm{o}}$ to $r_{\text {hit }}$ (see Appendix A.2). By equating the $r$-integral with the $\theta$-integral, we can then get the solution of $r_{\text {hit }}$ (see Appendix A.3). Once $r_{\text {hit }}$ is obtained, one can subsequently obtain the azimuthal position of the photon, $\phi_{\text {hit }}$, when $\theta=\theta_{\star}$ and $r=r_{\text {hit }}$, and the time needed $\delta t_{\text {hit }}=t_{\text {hit }}-t_{\mathrm{o}}$ for the photon propagating from $\left(t_{\text {hit }}, r_{\text {hit }}, \theta_{\star}, \phi_{\text {hit }}\right)$ to the observer (see Appendix A.4).

The trajectory of a photon with arbitrary $(\alpha, \beta)$ from the distant observer may not hit the surface of the star at any time $t_{\star}$ when $\theta=\theta_{\star}$. We adopt the following procedure to judge whether a photon can reach the surface of the star. In the pseudo-Cartesian coordinate system $x y z$, we have $\vec{R}_{\text {hit }}=\left(x_{\text {hit }}, y_{\text {hit }}, z_{\text {hit }}\right)$ and

$$
\left\{\begin{array}{l}
x_{\text {hit }}=r_{\text {hit }} \sin \theta_{\star} \cos \phi_{\text {hit }}, \\
y_{\text {hit }}=r_{\text {hit }} \sin \theta_{\star} \sin \phi_{\text {hit }}, \\
z_{\text {hit }}=r_{\text {hit }} \cos \theta_{\star},
\end{array}\right.
$$

according to Equation (17). The position of the star at any given time $t_{\star}$ can be described as $\vec{R}_{\star}=\left(x_{\star}, y_{\star}, z_{\star}\right)$, which are obtained similarly as $\vec{R}_{\text {hit }}$. The distance of the photon at $\theta=\theta_{\star}$ to the star in the $x y z$ system is defined as

$$
\begin{aligned}
d & =\left|\vec{R}_{\text {hit }}-\vec{R}_{\star}\right| \\
& =\sqrt{\left(x_{\text {hit }}-x_{\star}\right)^{2}+\left(y_{\text {hit }}-y_{\star}\right)^{2}+\left(z_{\text {hit }}-z_{\star}\right)^{2}} .
\end{aligned}
$$

We assume that a photon trajectory hits on the surface of the star if $d<\xi r_{\star}$ and $\xi=10^{-6}-10^{-8}$ In this case,

\footnotetext{
${ }^{4}$ For a star with mass $\sim 3 M_{\odot}$, its radius is $\sim 1.7 \times 10^{6} \mathrm{~km} \sim 0.3 \mathrm{r}_{\mathrm{g}}$ if $M_{\bullet}=4 \times 10^{6} M_{\odot}$. The setting of $\xi=10^{-6}-10^{-8}$ enables sufficient accuracy for relating the position of an example star to its image in the observer's sky plane, as we have $d \lesssim 0.05 r_{\mathrm{g}}$, substantially less than the star radius, even for the example star with the largest semimajor axis.
} 
$(\alpha, \beta)$ represent the apparent position of the image of the star at $\left(t_{\star}, r_{\star}, \theta_{\star}, \phi_{\star}\right)$ in the observer's sky plane, which are re-denoted as $\left(\alpha_{\star}, \beta_{\star}\right)$. The image $\left(\alpha_{\star}, \beta_{\star}\right)$ should be detected at a time $t_{\mathrm{o}}=t_{\star}+\delta t_{\mathrm{hit}}$. Therefore, the orbital motion of stars in the vicinity of the MBH represent by the change of $\alpha_{\star}$ and $\beta_{\star}$ in a time sequence of $t_{\mathrm{o}}$. In the meantime, the four-momentum of a photon with motion parameters $\lambda$ and $q$ at any position, e.g., $\mathbf{p}_{\text {hit }}=\left(p_{t, \text { hit }}, p_{r, \text { hit }}, p_{\theta, \text { hit }}, p_{\phi, \text { hit }}\right)$ at $\left(r_{\text {hit }}, \theta_{\star}, \phi_{\text {hit }}\right)$ and $\mathbf{p}_{\mathrm{o}}=\left(p_{t, \mathrm{o}}, p_{r, \mathrm{o}}, p_{\theta, \mathrm{o}}, p_{\phi, \mathrm{o}}\right)$ at $\left(R_{\mathrm{GC}}, i, 0^{\circ}\right)$, can be obtained by

$$
\left\{\begin{array}{l}
p_{t}=-E, \\
p_{r}= \pm E \sqrt{R} / \Delta, \\
p_{\theta}= \pm E \sqrt{\Theta}, \\
p_{\phi}=\lambda E,
\end{array}\right.
$$

in the Boyer-Lindquist coordinates. We can then obtain the redshift of the photon as

$$
Z=\frac{\mathbf{p}_{\text {hit }} \cdot \mathbf{u}_{\star}}{\mathbf{p}_{\mathrm{o}} \cdot \mathbf{u}_{\mathrm{o}}}-1=-\frac{\mathbf{p}_{\mathrm{hit}} \cdot \mathbf{u}_{\star}}{E_{\mathrm{o}}}-1 .
$$

Here $\mathbf{u}_{\circ}=(-1,0,0,0)$ and $\mathbf{u}_{\star}$ are the four-velocities of the distant observer and the star, respectively, $E_{\mathrm{o}}$ is the energy of the photon received by the observer. According to Equation (29), the redshift curve can be obtained for each star. This redshift can be measured by the shift of the center(s) of emission or absorption line(s).

In order to perform the ray-tracing calculations efficiently, the integrations are started from the point at $\left(10^{8} r_{\mathrm{g}}, i, 0\right)$ rather than $\left(R_{\mathrm{GC}}, i, 0\right)$. This setting does not affect the estimations of the impact parameters $\alpha$ and $\beta$ since the bending of light is not important anymore at a distance larger than $10^{8} r_{\mathrm{g}}$ from the $\mathrm{MBH}$. The final (mock) observations of the apparent positions of a star on the observer's sky plane should be given by $\alpha r_{\mathrm{g}} / R_{\mathrm{GC}}$ and $\beta r_{\mathrm{g}} / R_{\mathrm{GC}}$.

\section{APPARENT ORBITS AND REDSHIFT CURVES OF EXAMPLE STARS}

The apparent position of a star in the observer's sky and the redshift of the star at any given time can be obtained according to the procedure described in Sections 3 and 4 . We illustrate the effects of the $\mathrm{MBH}$ spin and its direction on the apparent orbits and redshift curves by using those example stars listed in Table円in this Section.

\subsection{Spin-induced Apparent Position Displacements}

Figure 3 shows the trajectory of each example star on the observer's sky, which is described by the right ascension (R.A.) and declination (Dec.) of the star with respect to the GC MBH (or Sgr A*). The initial settings for the orbital parameters of each star are listed in Table 1. As seen from Figure 3, the orbit of each star precesses mainly due to the advancement of periapsis; $\mathrm{Eb}$ has the most significant advance precession, while S0-102 has the least advance precession. The advance precession of periapsis of a star per orbit is

$$
\begin{aligned}
\delta \Upsilon_{\mathrm{S}}^{\prime} & =3 \pi \frac{2 r_{\mathrm{g}}}{a_{\text {orb }}\left(1-e_{\text {orb }}^{2}\right)} \\
& \simeq 0.19^{\circ}\left(\frac{4 \times 10^{6} M_{\odot}}{M_{\bullet}}\right)\left(\frac{10^{3} \mathrm{AU}}{a_{\text {orb }}}\right) \frac{1-0.88^{2}}{1-e_{\text {orb }}^{2}},(30)
\end{aligned}
$$

where $a_{\text {orb }}$ and $e_{\text {orb }}$ are the semimajor axis and eccentricity of the star in the Newtonian approximation (e.g., Misner et al. 1973). For those example stars listed in Table 1. $\delta \Upsilon_{S}^{\prime}$ are $0.19^{\circ}, 0.095^{\circ}, 0.64^{\circ}, 3.64^{\circ}, 2.39^{\circ}$, and $0.59^{\circ}$, respectively. Considering the projection of the orbital plane of each star to the observer's sky plane, the apparent shifts of the apoapsides of the example stars after three full orbits are $3 a_{\text {orb }}\left(1+e_{\text {orb }}\right) \delta \Upsilon_{\mathrm{S}}^{\prime}(1-$ $\left.\cos ^{2} \Upsilon^{\prime} \sin ^{2} I^{\prime}\right)^{1 / 2} \simeq 2.23,0.77,1.67,10.0,1.67$, and 0.29 mas for S0-2/S2, S0-102, Ea, Eb, Ec, and Ed, respectively (see Fig. 31). According to Figure 3, the advancement of periapsis can be accurately measured within a few orbits for all of the example stars, including S02/S2 and S0-102, if the astrometric precision can reach $100-10 \mu$ as scale. The spin-induced effects, including the Lense-Thirring precession and the frame dragging, cannot be clearly seen in Figure 3 because they are more than one order of magnitude smaller than the periapsis advancement, and these effects are addressed in Figures 4.8

If the MBH is non-spinning $(a=0)$, the trajectories of those example stars on the observer's sky plane at a given moment may slightly deviate from those shown in Figure 3 for the case with $a=0.99$. Figure 4 shows the evolution of this difference for each star over three full orbits. The difference in the apparent position is mainly due to the Lense-Thirring precession and the frame dragging. Figure 5 shows the distance $(\delta)$ between the apparent position of each star on the observer's sky plane at each given moment for the case with $a=0.99$ and that with $a=0$. After the motion of one full orbit of each star, the differences at apoapsis are $~ 8.7,1.6,4.7,92.5$, 11.1, and 2.6 $\mu$ as for S0-2/S2, S0-102, Ea, Eb, Ec, and Ed, respectively (see Fig. 5). These values are roughly consistent with simple analytical estimates as illustrated below. This difference mounts up with the increasing number of orbits as shown in Figures 4 and 5

The Lense-Thirring precession and the frame dragging cause the precession of the orbital plane of a star, which leads to a difference between the position of the star in the sky plane at apoapsis/periapsis for the case with a rapidly spinning $\mathrm{BH}$ and that with a non-spinning $\mathrm{BH}$ after one full orbit. For perturbation theory, this difference is approximately given by

$$
\begin{aligned}
\delta_{\text {apo } / \text { peri }} \simeq & a_{\text {orb }}\left(1 \pm e_{\text {orb }}\right)\left[\delta^{2} \Omega^{\prime}\left(1-\sin ^{2} \Upsilon^{\prime} \sin ^{2} I^{\prime}\right)+\right. \\
& \delta^{2} \Upsilon^{\prime}\left(1-\cos ^{2} \Upsilon^{\prime} \sin ^{2} I^{\prime}\right)+\sin \Upsilon^{\prime 2} \sin ^{2} I^{\prime} \delta^{2} I^{\prime}+ \\
& 2 \cos I^{\prime} \delta \Omega^{\prime} \delta \Upsilon^{\prime}-2 \sin \Upsilon^{\prime} \cos \Upsilon^{\prime} \sin I^{\prime} \cos I^{\prime} \delta \Upsilon^{\prime} \delta I^{\prime} \\
& \left.-2 \sin \Upsilon^{\prime} \cos \Upsilon^{\prime} \sin I^{\prime} \delta \Omega^{\prime} \delta I^{\prime}\right]^{1 / 2} .
\end{aligned}
$$

Here, the signs "+" and "-" are for the difference at apoapsis $\left(\delta_{\text {apo }}\right)$ and periapsis $\left(\delta_{\text {peri }}\right)$, respectively, $\delta \Omega^{\prime}$, $\delta \Upsilon^{\prime}$, and $\delta I^{\prime}$ are the changes of $\Omega^{\prime}, \Upsilon^{\prime}$, and $I^{\prime}$ due to the Lense-Thirring precession over one full orbit, respectively. For the detailed derivation of Equations 31 . see Appendix B According to Equation (31), we obtain $\delta_{\text {apo }}=8.6,1.5,4.4,62.5,8.4$, and $0.7 \mu$ as; $\delta_{\text {peri }}=0.55$, $0.30,0.28,0.64,0.55$, and $0.40 \mu$ as for the example stars S0-2/S2, S0-102, Ea, Eb, Ec, and Ed, respectively, if $a=0.99$ for the rapidly spinning $\mathrm{BH}$ case. The $\delta_{\text {apo }}$ values are roughly consistent with numerical results obtained above for most example stars, except that for Ed.

We note here that the $\delta$ at periapsides shown in Fig- 

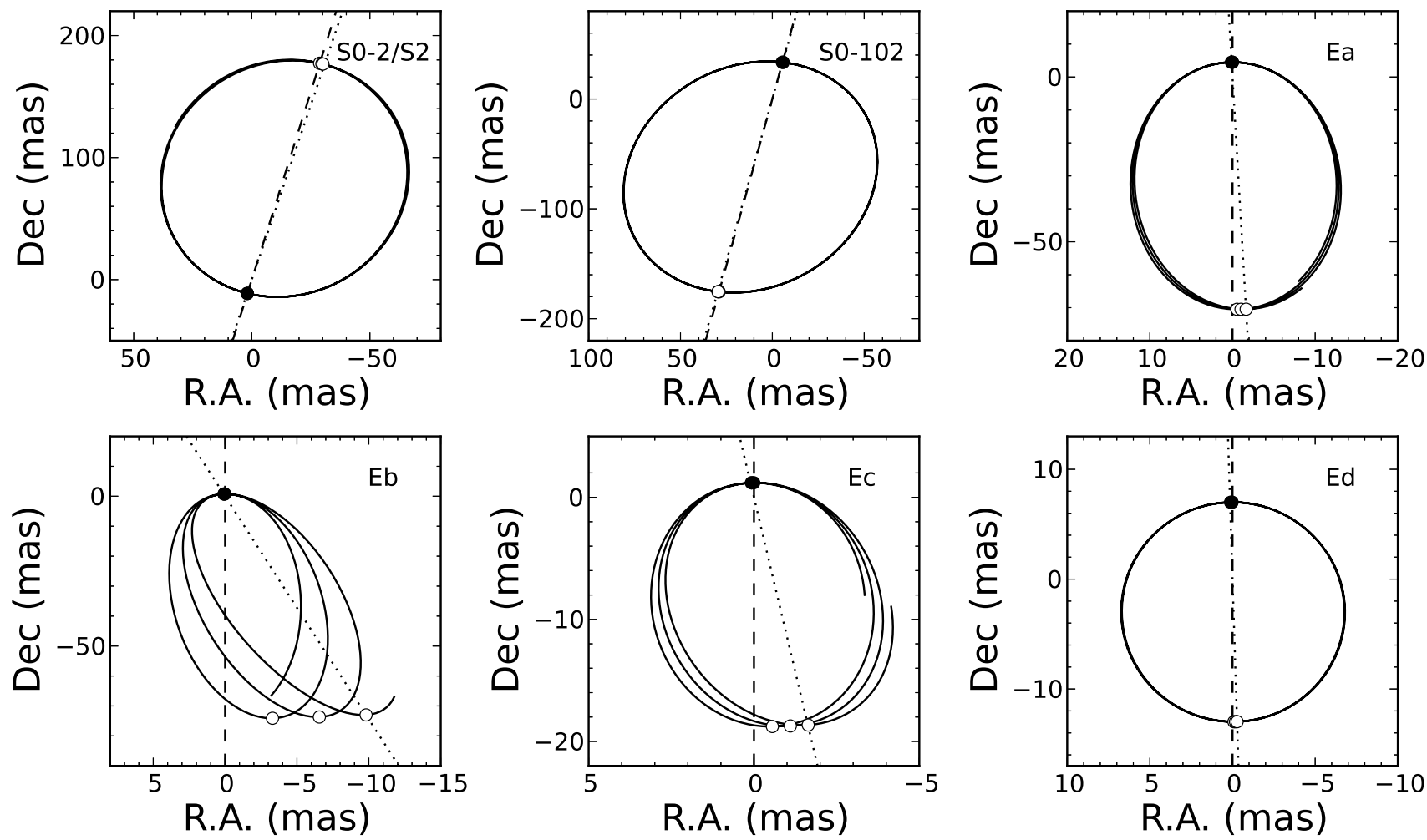

FIG. 3. - Evolution of the apparent position of each example star on the observer's sky plane. The apparent position of an example star is described by the R.A. and Dec. Panels from left to right, top to bottom, represent the example stars S0-2/S2, S0-102, Ea, Eb, Ec, and Ed, respectively. The initial settings of the orbital parameters for these stars are listed in Table 1 Three full orbits are shown for each example star. Open and solid circles mark the locations of the apoapsides and periapsides, respectively. In each panel, the dashed and dotted lines represent the eccentric vectors obtained by approximating the star orbit to a Newtonian ellipse for the first and the third orbit, respectively. As seen from the figure, the apparent orbital precession is most significant ( 3.1 mas per orbit) for the example star $\mathrm{Eb}$, which has the smallest pericenter distance.

ure 5 is obtained in a way slightly different from the $\delta_{\text {apo }}$ estimated from Equation (31). The values of $\delta$ are calculated for the projected distances at any given time between the star rotating around a rapidly spinning $\mathrm{MBH}$ $(a=0.99)$ and a star with the same initial orbital elements but rotating around a non-spinning $\mathrm{MBH}(a=0)$; while the value of $\delta_{\text {apo }}$ is estimated by considering the difference between the position differences of two adjacent apoapsides of a star rotating around an $\mathrm{MBH}$ with $a=0.99$ and those of a star with the same initial orbital elements rotating around a non-spinning $\mathrm{MBH}$. As the orbital periods of these two stars are slightly different, the shift of $\delta$ at two adjacent apoapsides shown in Figure 5 should therefore be roughly the same as, but different from, the analytical estimates $\delta_{\text {apo }}$, and the difference is most significant for those with small eccentricity.

For a consistency check between the numerical results obtained in this study and the analytical ones, as an example, we show the apoapsis shifts numerically estimated in two different ways for those stars with the same semimajor axis of $300 \mathrm{AU}$ (or $80 \mathrm{AU}$ ) but various eccentricities after one full orbit motion in Figure 6. The other initial orbital elements of those stars are the same as those of Ea (or Ec). The top panel of Figure 6 shows the numerical results obtained in the same way as $\delta_{\text {apo }}$, which are well consistent with the analytical estimates at $e \lesssim 0.8$, but higher than the analytical ones at $e \gtrsim 0.9$. There are three reasons for the difference at high eccentrici- ties: (1) the Newtonian approximation to the orbit of a star for the initial conditions becomes inaccurate if the star is close to the MBH (e.g., for those orbits with extremely high eccentricities); (2) for an Ea-like star with $e_{\text {orb }}$ higher than 0.98 , its orbital precession can be larger than $\sim 20^{\circ}$ per orbit, therefore, the first order approximation (for the projection) to obtain Equation (31) is inaccurate; (3) the contributions from the higher order precessions are ignored in the analytical estimates. As showed in Figure 6, the combinations of the above three factors can almost lead to $20 \%$ to a factor of two difference when $e_{\text {orb }} \gtrsim 0.98$. The bottom panel of Figure 6 shows the numerical results of $\delta$ obtained in the same way as those shown in Figure 5, which apparently differ significantly from the analytical estimates at both $e_{\text {orb }} \lesssim 0.7$ and $e_{\text {orb }} \gtrsim 0.9$ because $\delta$ at apoapsis and $\delta_{\text {apo }}$ are defined and obtained in a slightly different way.

According to Figure 5, we conclude that, if the accuracy in determining the apparent positions of any of those example stars (except S0-102) on the sky plane can reach $\lesssim 10 \mu \mathrm{as}$, the spin of the $\mathrm{GC} \mathrm{MBH}$ can be then constrained by fitting the evolution of its positions over several orbits as demonstrated by a Bayesian fitting method in Section 6] (see also Tab. 21).

For a star with given initial orbital elements, the displacement of apoapsis after one full orbit due to the spin effects is proportional to the absolute value of the spin, and it also depends on the spin direction (see eqs. B5. B8). 

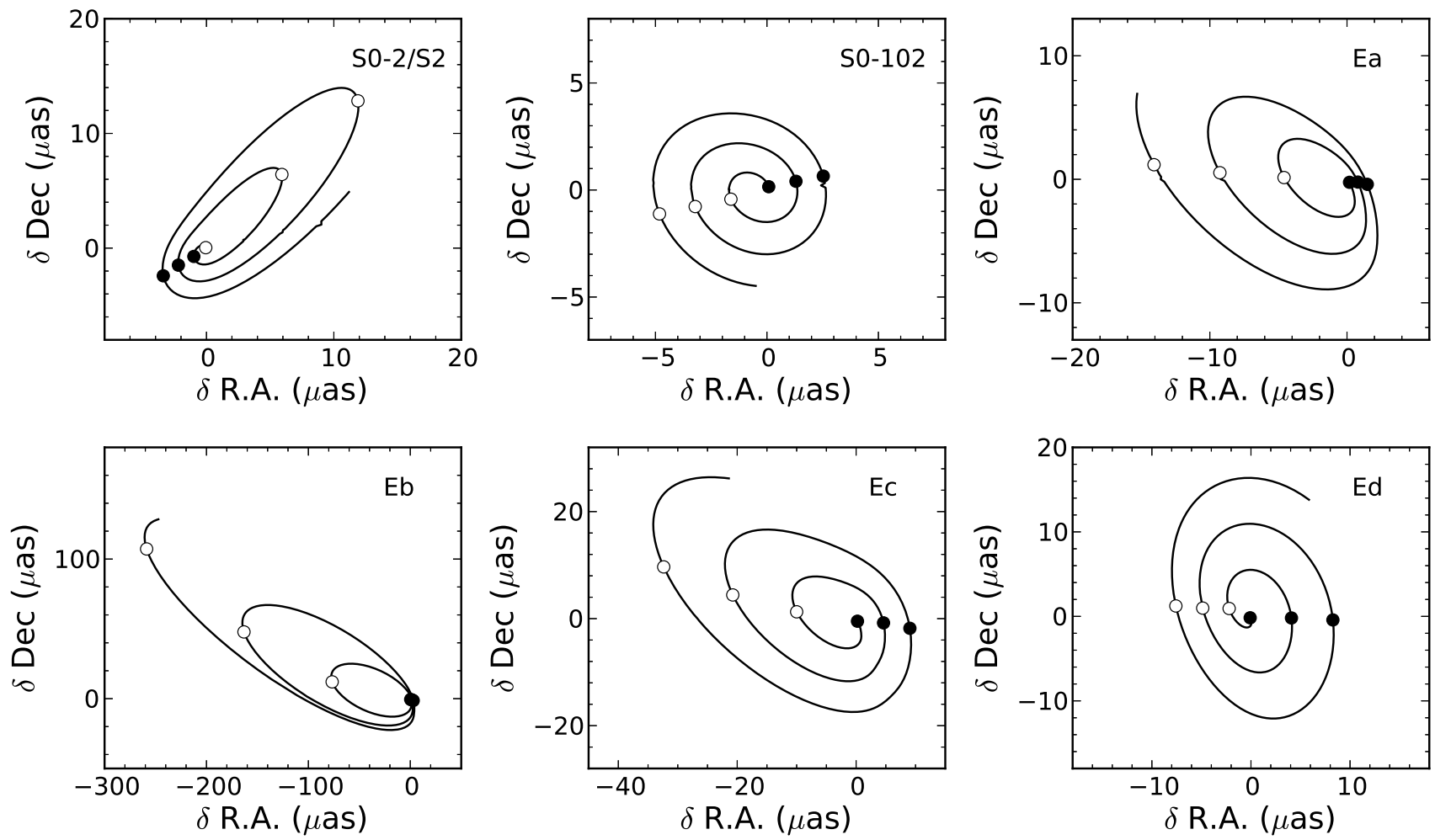

FIG. 4.- Difference between the apparent position of each example star on the observer's sky plane at each given moment for the case with a rapidly spinning central MBH $(a=0.99)$ and that with a non-spinning MBH $(a=0)$. The differences in R.A. and Dec. are denoted as $\delta$ R.A., and $\delta$ Dec., respectively. Panels from left to right, top to bottom, represent the example stars S0-2/S2, S0-102, Ea, Eb, Ec, and Ed, respectively. Three total orbits for each star are shown here and the first orbit of each star starts at $(\delta \mathrm{R} . \mathrm{A} ., \quad \delta \mathrm{Dec})=.(0,0)$. Solid and open circles mark the periapsis and apoapsis passage points, respectively. This figure shows that the difference in the apparent position of each example star increases with increasing time because of the Lensing-Thirring precession and the frame dragging and other higher order GR effects induced by the MBH spin in the rapidly spinning MBH case.

To illustrate the dependence of the spin-induced position displacement on the spin direction, the top panel of Figure 7 shows the evolution of $\delta$ of Ea for three different spin directions. The $\mathrm{MBH}$ spin is fixed at $a=0.99$. As seen from this figure, $\delta$ is the most significant when $(i, \epsilon)=\left(72^{\circ}, 90^{\circ}\right)$, and the least significant when $(i, \epsilon)=\left(159^{\circ}, 63^{\circ}\right)$. The difference in the spin direction may lead to more than an order of magnitude difference in the maximum position displacement over a full orbit, which suggests that the MBH spin may be easier to be constrained by the relativistic motion of a given star if the spin vector is close to some special direction. We note here that $\delta_{\text {apo }}$ of Ea is the largest $\left(\delta_{\text {apo }} \simeq 20 \mu \mathrm{as}\right.$ for $a=0.99)$ if $(i, \epsilon)=\left(72^{\circ}, 90^{\circ}\right)$ or $\left(108^{\circ}, 270^{\circ}\right)$, and it is almost the smallest $\left(\delta_{\text {apo }} \simeq 0.4 \mu\right.$ as for $a=0.99$ ) if $(i, \epsilon)=\left(159^{\circ}, 63^{\circ}\right)$ or $\left(21^{\circ}, 243^{\circ}\right)$. (These values are obtained from Equation 31.)

The spin-induced (apoapsis) position displacement $(\delta)$ also depends on the orientation of the orbital plane of a star relative to the sky plane, if the $\mathrm{MBH}$ spin and its direction are fixed (see eqs. B5, B8 $)$. The bottom panel of Figure 7 illustrates this dependence by plotting the evolution of $\delta$ for Ea and two other stars with the same semimajor axis and eccentricity as those of Ea but with different orbital orientations. The $\mathrm{MBH}$ spin is fixed at $a=0.99$ and the spin direction is $(i, \epsilon)=\left(45^{\circ}, 180^{\circ}\right)$. As seen from the figure, the amplitude of $\delta$ may be different by more than an order of magnitude and the evolution pattern of $\delta$ can also be different if the orientation of the star orbital plane is different, simply because of the projection effect.

The apparent positions of a star, rotating around the $\mathrm{MBH}$, on the observer's sky plane, may be significantly affected by the bending of light rays propagating from the star to the observer due to the relativistic potential of the MBH. Figure 8 shows the difference in the apparent position of each example star at each moment for the case with, and without, the consideration of the bending of light (obtained from the ray-tracing method detailed in Appendix A . As seen from this figure, the position difference can be as large as 10-20 $\mu$ as, roughly on the order of the Einstein radius of the $\mathrm{MBH}\left(\sim 4 G M_{\bullet} / c^{2} \sim 20 \mu \mathrm{as}\right)$, which must be accurately considered when constraining the $\mathrm{MBH}$ spin, as the spin-induced position displacements are mostly on the same order (see Fig 5). The position displacements caused by the bending of light are almost the same for the case with a rapidly spinning $\mathrm{MBH}$ and the case with a non-spinning MBH, and thus cannot be distinguished from each other (see the solid lines for cases with $a=0.99$ in Figure 8 , the results obtained for cases with $a=0$ coincide with those solid lines, and the differences between these two cases are on the order of $0.1 \mu \mathrm{as})$. These differences, though small, are roughly on the same order as the effects induced by the quadruple moment of the $\mathrm{MBH}$ for $\mathrm{S} 0-2 / \mathrm{S} 2$. Therefore, it is important to include them for future high-precision 

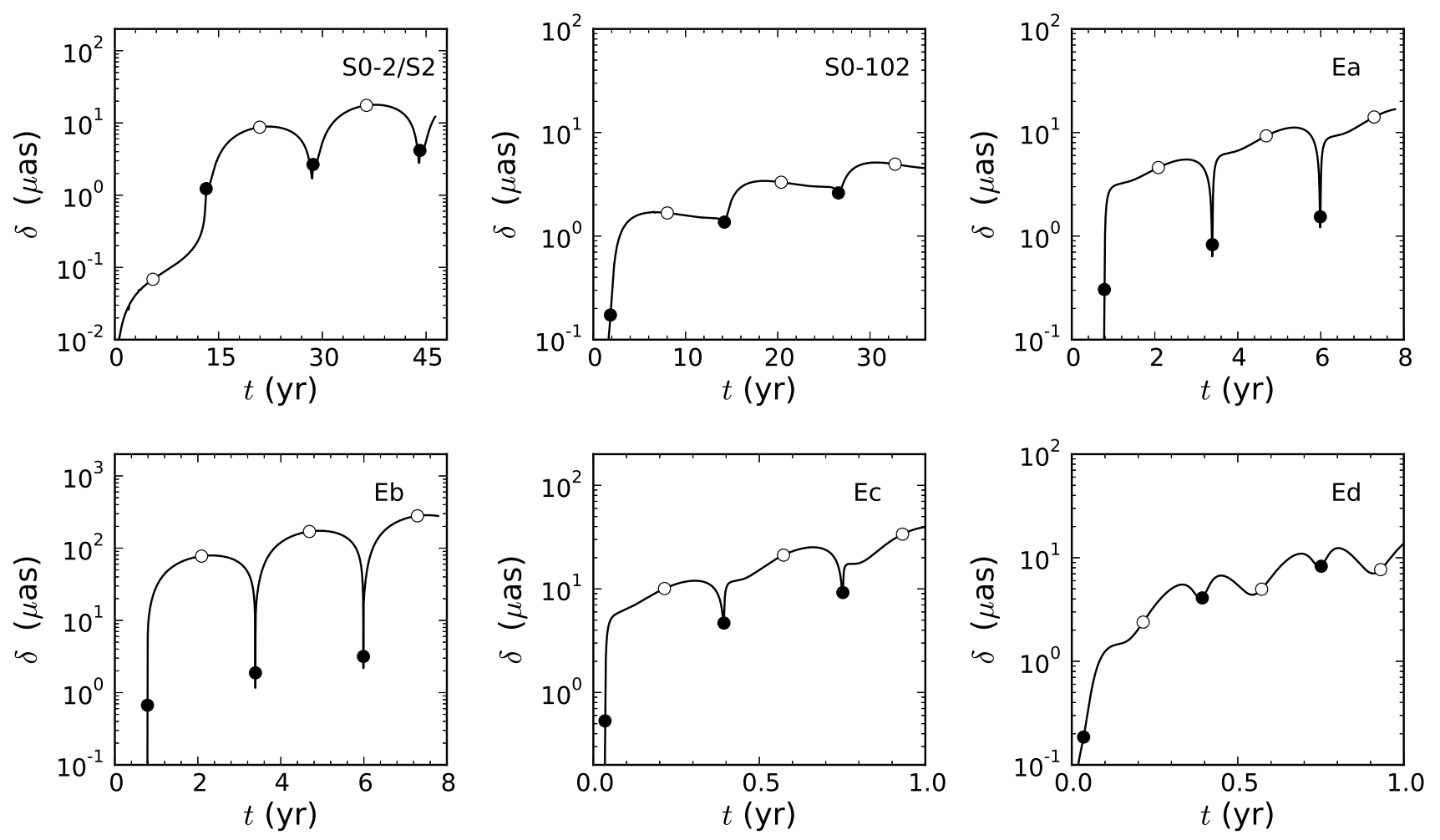

FIG. 5.- Distance between the apparent position of each example star on the observer's sky plane at each given moment for the case with a rapidly spinning central MBH $(a=0.99)$ and that with a non-spinning MBH $(a=0)$. The distance is defined as $\delta=\sqrt{(\delta \mathrm{R} . \mathrm{A} .)^{2}+(\delta \mathrm{Dec})^{2}}$, and $\delta$ R.A. and $\delta$ Dec. are shown in Figure 4 Panels from left to right, top to bottom, represent the example stars S0-2/S2, S0-102, Ea, Eb, Ec, and Ed, respectively. Open and solid circles mark the locations of the apoapsides and periapsides, respectively. Three total orbits for each example star are shown here and the first orbit of each star starts at $t=0$.

measurements that may be used to constrain the quadruple moment and test GR. The cardioid-like shapes of the curves shown in Figure 8 are due to the position change of the star with respect to the central $\mathrm{MBH}$, and consequently the change of the $\mathrm{MBH}$ potential and the deflection angle. If the true position of the star is right in between the $\mathrm{MBH}$ and the observer, the apparent position should be at the coordinate origin in Figure 8 . We note that the trajectories of apparent positions of a distant star deflected by Jupiter, which is moving around sun, have similar cardioid-like shapes due to similar underlying physics (see Kopeikin \& Makarov 2007).

\subsection{Spin-induced Redshift Differences}

Figure 9 shows the redshift evolution of each example star monitored by a distant observer for three full orbits. As shown in this figure, the maximum spin-induced redshift (or blueshift) differences over a full orbit are about $0.4,0.05,2.28,82,55$, and $3.7 \mathrm{~km} \mathrm{~s}^{-1}$ for $\mathrm{S} 0-2 / \mathrm{S} 2$, S0102, Ea, Eb, Ec, and Ed, respectively.

The redshift of a star is approximately given by

$$
Z \simeq-\mathcal{V}_{z^{\prime}} \simeq-\mathcal{V}_{\mathrm{K}, z^{\prime}}+\frac{c}{2}\left(\mathcal{V}_{\mathrm{K}} / c\right)^{2}+c r_{\mathrm{g}} / r+\delta \mathcal{V}(a)
$$

with the first, second, third, and fourth terms in the right-hand side representing the Newtonian Doppler shift due to the Keplerian motion of the star, the special relativity correction to the Doppler shift (or the transverse Doppler shift), the gravitational redshift, and the part of redshift due to the $\mathrm{MBH}$ spin, respectively. Here the redshift $Z$ is defined to have the same unit $\left(\mathrm{kms}^{-1}\right)$ as that of velocity. In the Newtonian approximation,

$$
\begin{gathered}
\mathcal{V}_{\mathrm{K}, z^{\prime}}=-\sqrt{\frac{G M_{\bullet}}{a_{\mathrm{orb}}\left(1-e_{\mathrm{orb}}^{2}\right)}} \sin I^{\prime}\left[e_{\mathrm{orb}} \cos \Upsilon^{\prime}+\cos \left(\Upsilon^{\prime}+v^{\prime}\right)\right], \\
\mathcal{V}_{\mathrm{K}} \simeq\left(2 G M_{\bullet} / r-G M_{\bullet} / a_{\mathrm{orb}}\right)^{1 / 2} \\
r \simeq a_{\mathrm{orb}}\left(1-e_{\mathrm{orb}}^{2}\right) /\left(1+e_{\mathrm{orb}} \cos v^{\prime}\right),
\end{gathered}
$$

and $\delta \mathcal{V}(a)$ is negligible comparing with those other terms. The second and third terms on the right side of Equation (32) are much less than the first term when $r \gg r_{\mathrm{g}}$. According to Equation (32), $\mathcal{V}_{z^{\prime}}$ approaches the maximum redshift and the maximum blueshift (or the maximum blueshift and the maximum redshift) at $v^{\prime} \sim \Upsilon^{\prime}$ and $\pi-\Upsilon^{\prime}$ if $\sin I^{\prime} \cos \Upsilon^{\prime}>0$ (or if $\sin I^{\prime} \cos \Upsilon^{\prime}<0$ ), respectively. At $v^{\prime}=\Upsilon^{\prime}$ (or $v^{\prime}=\pi-\Upsilon^{\prime}$ ), the star crosses the sky plane $\left(x^{\prime} y^{\prime}\right)$. As seen from Figure 9, the approximations given by Equation (32) are well consistent with the full GR calculations.

The gravitomagnetic field generated by the spinning $\mathrm{MBH}$ influences not only the motion of a star close to it but also the propagation of photons from the star to the distant observer (i.e., Thorne 1986). Both of these effects are encoded in the amount of shifts (or the redshift) of lines in the star spectrum measured at different times 5 Figure 10 shows the redshift difference $(\delta Z)$ of

\footnotetext{
${ }^{5}$ The low-order effects, such as the GR gravitational redshift, the
} 


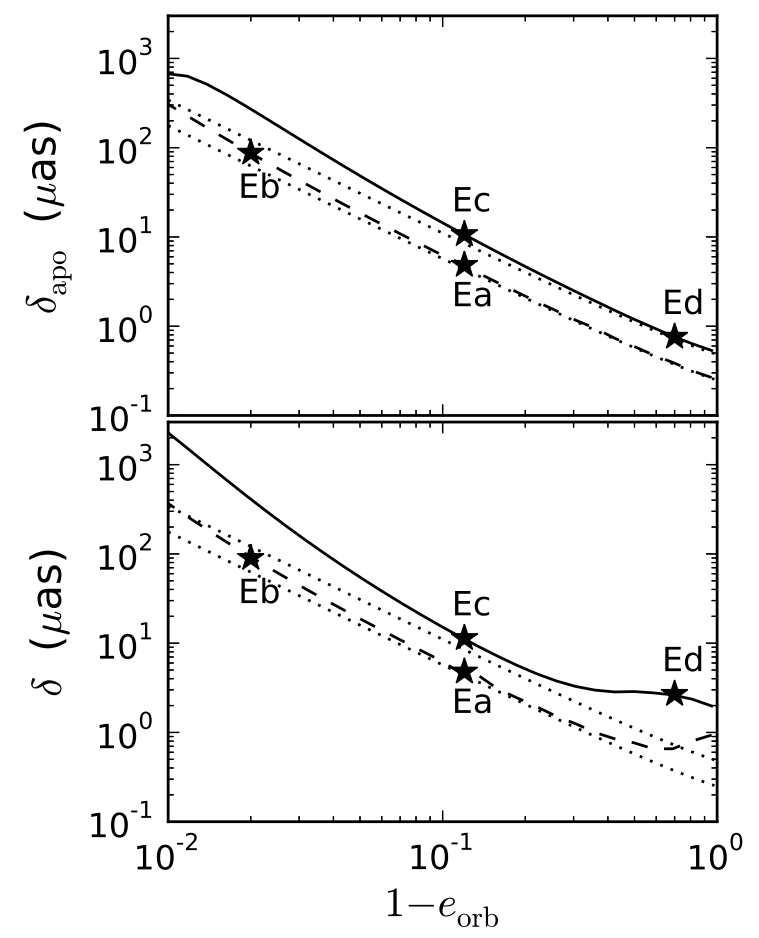

FIG. 6.- Top panel: projected apoapsis displacements due to the $\mathrm{MBH}$ spin after the motion of one full orbit. The solid (dashed) line represents the results of apoapsis shift defined in the same way as $\delta_{\text {apo }}$ for those stars having the same initial orbital elements as those of Ec (Ea), but with different eccentricities; the spin and its direction of the $\mathrm{MBH}$ is fixed at $a=0.99$ and $(i, \epsilon)=\left(45^{\circ}, 0^{\circ}\right)$. Dotted lines represent the analytical estimates of $\delta_{\text {apo }}$ for those stars by using Equation (31). Bottom panel: solid line (dashed line) represents the difference of $\delta$ between two apoapsides of two stars with the same initial orbital elements as those of Ec (Ea), with one of the two rotating around a rapidly spinning $\mathrm{MBH}(a=0.99)$ and the other around a non-spinning MBH $(a=0)$. Here $\delta$ is defined in the same way as that shown in Figure (5) at apoapsis. The dotted lines are the same as those in the top panel. The star symbols mark the location of the star Ea, Eb, Ec, and Ed, respectively.

each example star at each given moment for the case with a rapidly spinning $\mathrm{MBH}(a=0.99)$ and that with a non-spinning MBH. For each star, as seen from this figure, the redshift difference changes over the course of a single orbit, and it is most significant near the periapsis and most insignificant near the apoapsis; the amplitude of the differences also increases with increasing number of the orbital periods passed. For S0-2/S2, the redshift difference is about $\sim 0.3 \mathrm{~km} \mathrm{~s}^{-1}$ at the periapsis of its first orbit, and it mounts up to $\sim 1 \mathrm{~km} \mathrm{~s}^{-1}$ after about three full orbits; for the $\mathrm{Eb}$ and $\mathrm{Ec}$, the redshift difference can be up to $\gtrsim 100 \mathrm{~km} \mathrm{~s}^{-1}$ at the periapsis of the third orbit. The amplitude of the redshift differences is the largest for $\mathrm{Eb}$ and the smallest for S0-102, which is fully consistent with the dependence of the redshift difference due to the Lense-Thirring precession and the

relativistic Doppler shift, and the Rømer delay, on the redshift have been investigated in the literature separately through perturbative approximations (e.g., Zucker et al.|2006; Angélil \& Saha| 2010). In this study, we only focus on the spin related line shifts (or redshift) and do not separately study those low-order effects one by one as in previous studies. The low-order effects, as well as the high-order effects, are automatically involved in our full GR calculations.

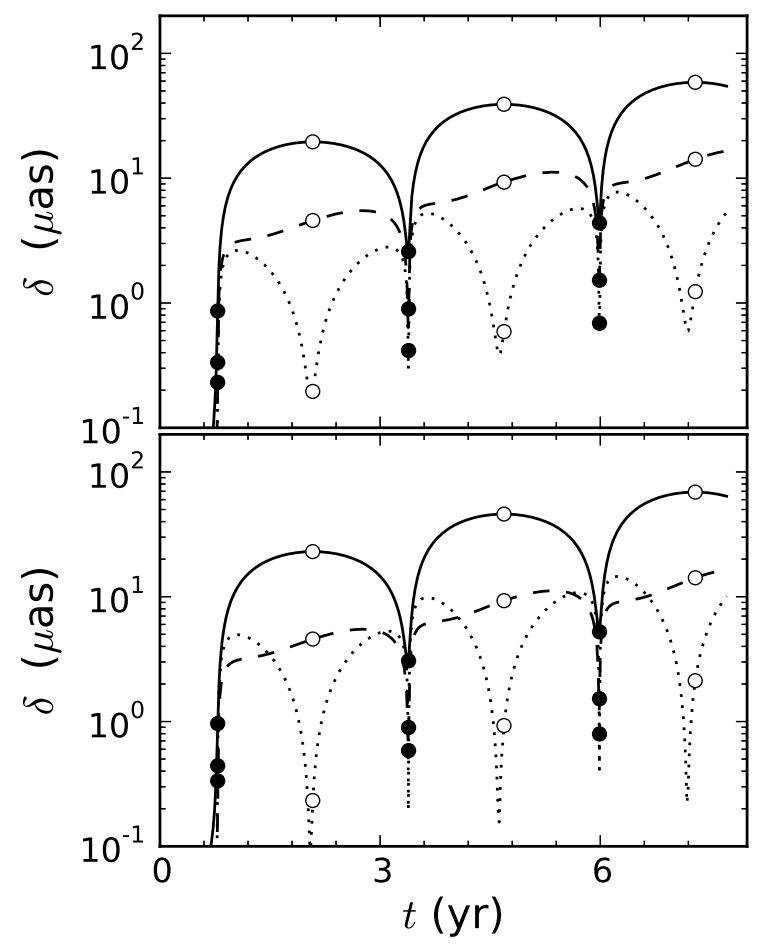

FIG. 7.- Dependence of the spin-induced position displacements on the spin direction and the star orbital orientation. The top panel shows the distance at any given moment between the apparent position of a star rotating around an $\mathrm{MBH}$ with $a=0.99$ and that of the star around an MBH with $a=0$, and these two stars have the same initial orbital elements as those of Ea. The solid, dashed, and dotted lines represent the cases for the MBH with a spin direction of $(i, \epsilon)=\left(71^{\circ}, 90^{\circ}\right),\left(45^{\circ}, 180^{\circ}\right)$, and $\left(159^{\circ}, 63^{\circ}\right)$, respectively. The bottom panel shows the distance at any given moment between the apparent position of a star rotating around an MBH with $a=0.99$ and that of a star rotating around an MBH with $a=0$; and these two stars have the same semimajor axis, eccentricity, and argument of periapsis as those of Ea, but with different inclination $\left(I^{\prime}\right)$ and longitude of the ascending node $\left(\Omega^{\prime}\right)$. The solid, dashed, and dotted lines represent for the cases with $\left(\Omega^{\prime}, I^{\prime}\right)=\left(22^{\circ}, 90^{\circ}\right),\left(45^{\circ}, 0^{\circ}\right)$, and $\left(73^{\circ}, 62^{\circ}\right)$, respectively. The spin direction of the spinning $\mathrm{MBH}$ is fixed at $(i, \epsilon)=\left(45^{\circ}, 0^{\circ}\right)$. Three total orbits are shown here and the first orbit starts at $t=0$.

frame dragging effect on the distance $(r)$ to the $\mathrm{MBH}$ revealed by Kannan \& Saha (2009) and Angélil \& Saha (2010), i.e., $\delta Z \propto r^{-2}$ [and $\propto a_{\text {orb }}^{-2}\left(1-e_{\text {orb }}\right)^{-2}$ at the periapsis]. If the accuracy of the redshift measurements can reach $\lesssim 1 \mathrm{~km} \mathrm{~s}^{-1}$ (or $\lesssim 50 \mathrm{~km} \mathrm{~s}^{-1}$ ), the spin of the MBH may be well constrained by using an observational redshift curve of S0-2/S2 (or the example star Eb or Ec) over three or more orbits and correspondingly $\gtrsim 45$ years (or $\gtrsim 6$ years for $\mathrm{Eb}$, or $\gtrsim 1$ years for $\mathrm{Ec}$ ).

The Lense-Thirring precession and the frame dragging can lead to a change in the orbital orientation of a star, as shown above and in Appendix B. One significant component of the redshift difference $\delta \mathcal{V}(a)$ must be that introduced by the change of the orbital orientation, i.e.,

$$
\begin{aligned}
\delta Z(v) \simeq & -\sqrt{\frac{G M_{\bullet}}{a_{\mathrm{orb}}\left(1-e_{\mathrm{orb}}^{2}\right)}}\left\{\cos I^{\prime}\left[e_{\mathrm{orb}} \cos \Upsilon^{\prime}+\cos \left(\Upsilon^{\prime}+v^{\prime}\right)\right]\right. \\
& \left.\times \delta I^{\prime \prime}+\sin I^{\prime}\left[-e_{\mathrm{orb}} \sin \Upsilon^{\prime}-\sin \left(\Upsilon^{\prime}+v^{\prime}\right)\right] \delta \Upsilon^{\prime \prime}\right\},(36)
\end{aligned}
$$

where $\delta I^{\prime \prime}$ and $\delta \Upsilon^{\prime \prime}$ are the changes of $I^{\prime}$ and $\Upsilon^{\prime}$ induced 

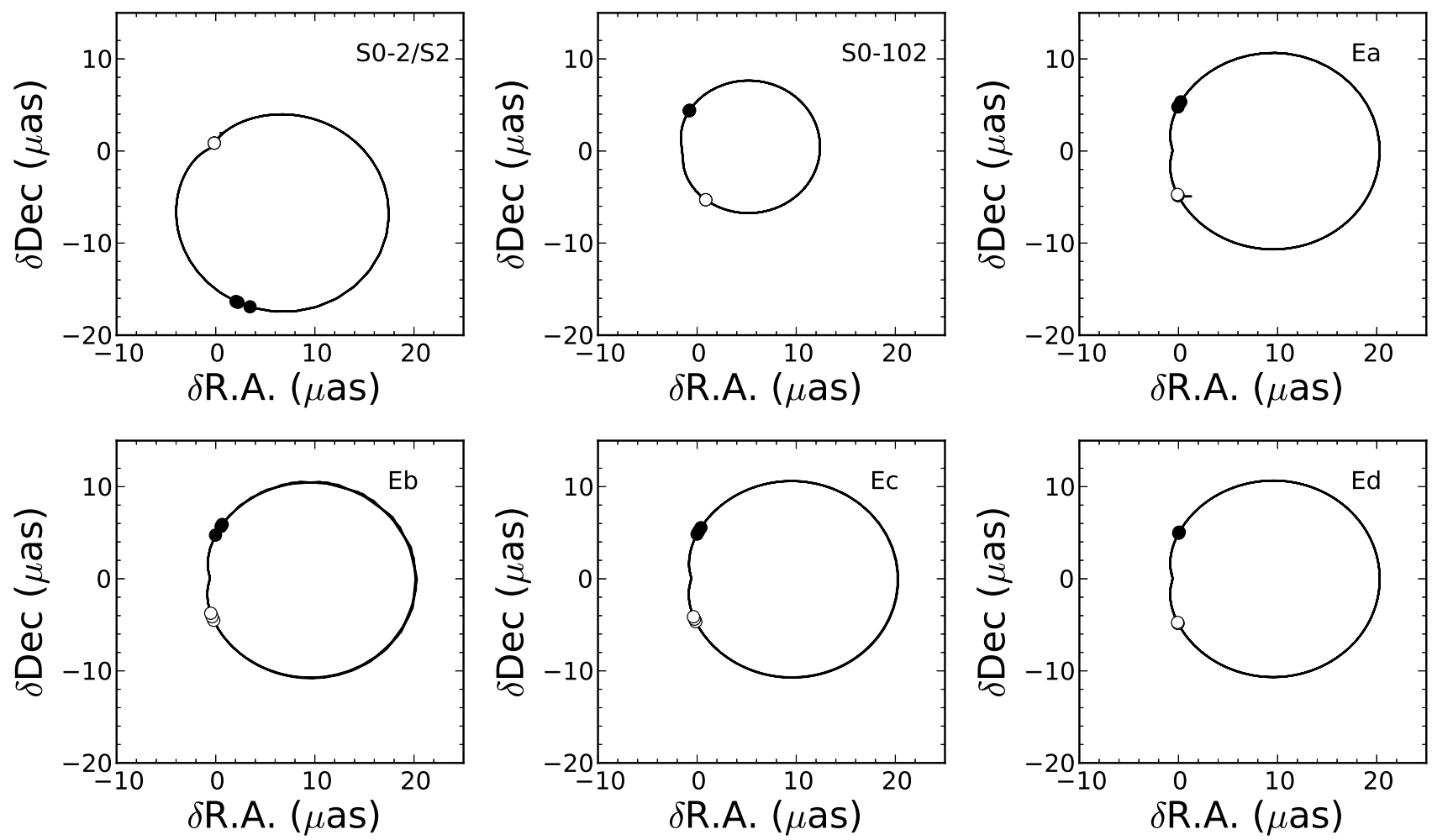

FIG. 8. - Differences in the apparent position of each example star at each given moment for the case with, and without, the consideration of the bending of light (obtained from the ray tracing technique). Panels from left to right, top to bottom, represent the example stars S0-2/S2, S0-102, Ea, Eb, Ec, and Ed, respectively. The open and solid circles mark the locations of the apoapsides and periapsides, respectively. Three total orbits for each example star are shown in this figure, but they are difficult to be distinguished.

by the Lense-Thirring effect since the start of the orbital motion from $v_{0}^{\prime}$ to $v^{\prime}$ (or $E_{0}$ to $E$ from $t_{0}$ to $t$ ). Furthermore,

$$
\begin{gathered}
\delta I^{\prime \prime} \simeq\left[\left(E-E_{0}\right)-e_{\text {orb }}\left(\sin E-\sin E_{0}\right)\right] \delta I^{\prime} / 2 \pi, \\
\delta \Upsilon^{\prime \prime} \simeq\left[\left(E-E_{0}\right)-e_{\text {orb }}\left(\sin E-\sin E_{0}\right)\right] \delta \Upsilon^{\prime} / 2 \pi, \\
E_{0}-e_{\text {orb }} \sin E_{0}=\sqrt{\frac{G M_{\bullet}}{a_{\text {orb }}^{3}}}\left(t_{0}-T_{0}\right), \\
E-e_{\text {orb }} \sin E=\sqrt{\frac{G M_{\bullet}}{a_{\text {orb }}^{3}}}\left(t-T_{0}\right) .
\end{gathered}
$$

Here $\delta I^{\prime}$ and $\delta \Upsilon^{\prime}$ are the spin-induced changes of $I^{\prime}$ and $\Upsilon^{\prime}$ per orbit (see Appendix B), $T_{0}$ is the time of pericentric passage with respect to the year of 2000, and $t_{0}=2020$ year is the starting time. The dashed lines in Figure 10 represent the analytical estimates obtained from Equation (36), which seem to be roughly consistent with the numerical results (dashed lines) obtained from the full GR calculations, at least at the apoapsides and the periapsides. According to Equation (36) and Appendix $\mathrm{B}, \delta Z$ is roughly proportional to $a_{\text {orb }}^{-2}\left(1-e_{\text {orb }}\right)^{-2}$ [or $a_{\text {orb }}^{-2}\left(1-e_{\mathrm{orb}}\right)^{-1}$ ] at periapsis [or apoapsis], if $1-e_{\mathrm{orb}} \ll$ 1. According to Equation (32), the maximum redshift (or blueshift) difference induced by the $\mathrm{MBH}$ spin at periapsis are $0.2(-0.06), 0.04(-0.02), 0.9(-2.0), 28(-68), 13$ $(-28)$ and $1.0 \mathrm{~km} \mathrm{~s}^{-1}\left(-1.4 \mathrm{~km} \mathrm{~s}^{-1}\right)$ over a full orbit for S0-2/S2, S0-102, Ea, Eb, Ec, and Ed, respectively, which are roughly consistent with the full GR numerical results shown in Figure 10. There are indeed some differences between the approximations given by Equation (36) and those obtained from the full GR calculations, which are due to (1) the adoption of the mean change rates of $\Omega^{\prime}$, $\Upsilon^{\prime}$, and $I^{\prime}$ (for the purpose of illustration here, we do not intend to adopt the evolution forms of those change rates as functions of $v^{\prime}$ ); and (2) the neglect of the light propagation effects and high-order precession in the approximations and the slight differences in the estimates of $\delta Z$ (as discussed for the position displacements above; see Fig. 6).

Figure 11 shows the maximum redshift difference (top panel) and the maximum blueshift difference (bottom panel) induced by the MBH spin $(\delta Z)$ obtained for stars with the same orbital elements as those of Ec (or Ea) except with various initial eccentricities. With an increasing number of orbits, the absolute value of the maximum redshift (or blueshift) difference mounts up. As seen from this figure, the analytical approximations are quite consistent with the numerical results, especially when the semimajor axis of the star is larger, which suggests that the main contribution to the redshift difference $\delta Z$ is the change of the star orbital orientation and thus the change of the projection of the velocity to the line of sight. For stars with small semimajor axes, the analytical estimates deviate from the full GR numerical results, which strengthens the necessity of using the full GR calculations for accurately constraining the $\mathrm{MBH}$ spin.

Note that the redshift difference due to the spin effects on the propagation of photons is about $10 \sim 100$ times smaller than that on the star orbit (see also 

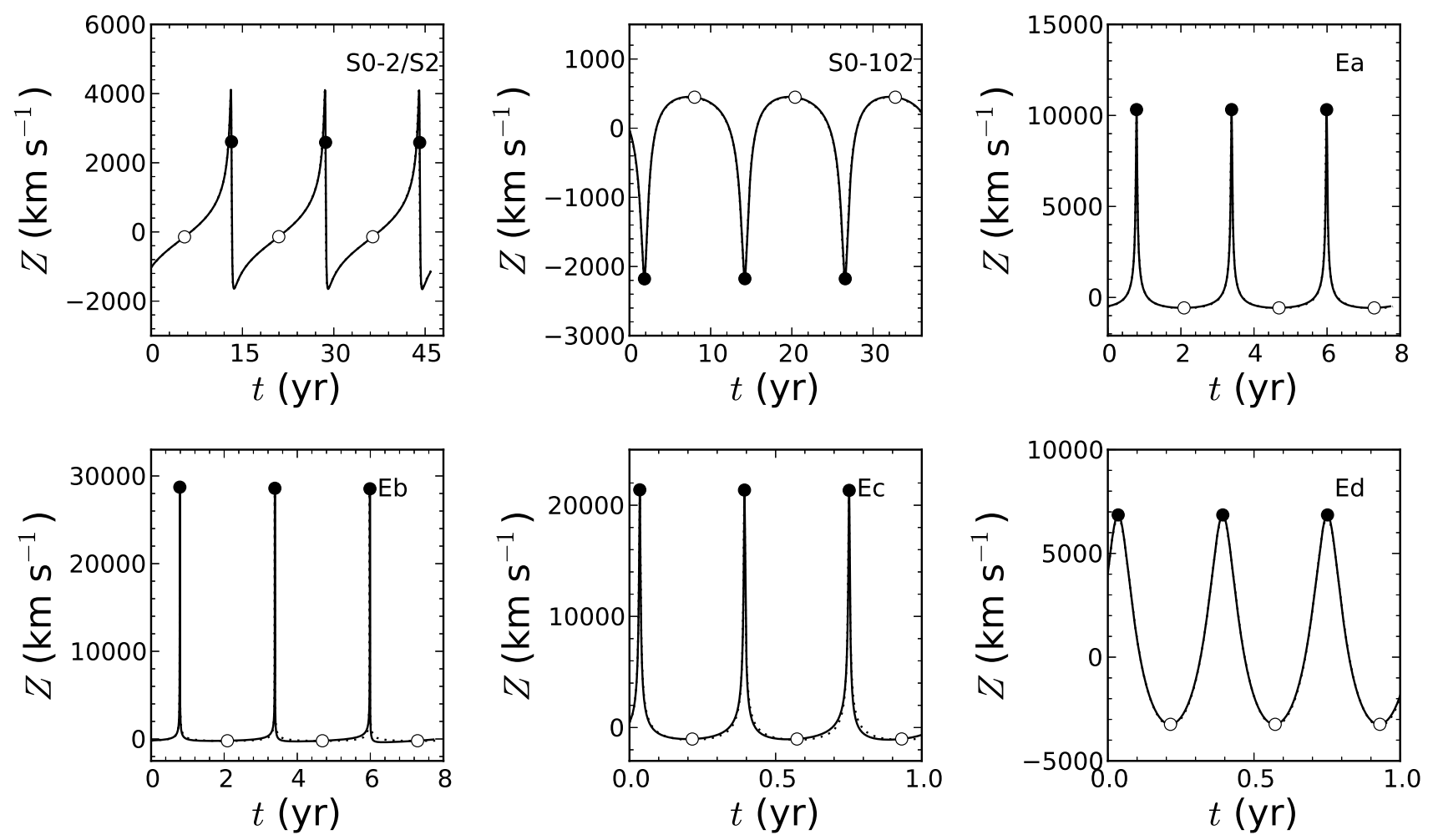

FIG. 9. - Redshift evolution curves of example stars. Panels from left to right, top to bottom, represent the example stars S0-2/S2, S0-102, Ea, Eb, Ec, and Ed, respectively. In each panel, the solid (open) circles mark the pericenter (apocenter) passage of the star, and the solid and dashed lines represent the numerical results and the approximation given by eq. (32), respectively. Totally three full orbits are shown here and the first orbit starts at $t=0$.

Angélil \& Saha 2010). For S0-2/S2, if the accuracy of redshift measurements is on the order of $\gtrsim 1 \mathrm{kms}^{-1}$, the spin effect on photon propagation may be negligible (although the Schwarzschild effect on photon propagation is significant, close to $10 \mathrm{~km} \mathrm{~s}^{-1}$, and cannot be neglected; see Angélil \& Saha 2010). However, for stars with smaller semimajor axes and higher eccentricities, such as the example stars Eb and Ec, it is necessary to include the spin effect on photon propagation (on the order of $\sim 3 \mathrm{~km} \mathrm{~s}^{-1}$ for $\mathrm{Eb}$ and $\sim 1 \mathrm{~km} \mathrm{~s}^{-1}$ for Ec, close to $10 \%$ of the total spin-induced redshift differences) in order to get an accurate constraint on the $\mathrm{MBH}$ spin. Our full GR calculations are fast and efficient, which enable us to automatically have the high-order precession effects due the MBH spin and thus provide a more accurate fitting scheme for future high-precision measurements (see Section 6) compared with those approaches adopting the perturbative approximations (e.g., Angélil et al. 2010).

The spin-induced redshift difference $(\delta Z)$ also depends on the spin direction and the orbital orientation of the star as shown in Figure 12. similar to that for the spininduced position displacement shown in Figure 8. The top panel of Figure 12 illustrates the dependence of $\delta Z$ on the spin direction by showing the evolution of $\delta Z$ of Ea, for three different spin directions. The bottom panel of Figure 12 illustrates the dependence of $\delta Z$ on the orbital orientation of stars, by showing the evolution of $\delta Z$ of Ea and two other stars with the same semimajor axis and eccentricity as those of Ea but with different orbital orientations. As seen from this figure, these dependences help to simultaneously constrain both the absolute spin value and the spin direction.

\section{A GENERAL METHOD TO CONSTRAIN THE MBH SPIN PARAMETER}

In this section, we introduce a general method to constrain the spin parameter and the metric of the $\mathrm{GC} \mathrm{MBH}$ via the relativistic motion of a star in the immediate vicinity of the MBH by using the MCMC technique.

We first generate mock observations for each example star listed in Table [1] in order to investigate whether the spin parameter of the GC MBH can be tightly constrained by monitoring the orbital motion of a close-in star through the next generation facilities, such as the GRAVITY on VLTI, TMT, and E-ELT. For this purpose, we assume that the MBH mass is $M_{\bullet}=4 \times 10^{6} M_{\odot}$ and the distance from the Sun to the GC is $R_{\mathrm{GC}}=8 \mathrm{kpc}$. The MBH spin and its direction are assumed as those listed in Table 1]. With each given set of initial parameters for the six orbital elements of a star, we can obtain the trajectory of the star's apparent position on the sky plane $\left(\alpha_{\mathrm{obs}, j}, \beta_{\mathrm{obs}, j}\right)$ and the redshift curve $\left(Z_{\mathrm{obs}, j}\right)$ for any given time period through the full general relativistic calculations described in Sections 3 and 4 Here the subscript $j$ denotes a mock observation made at the observer's time $t_{\mathrm{obs}, j}$. The spin-induced position displacement is most significant near the apoapsis and the spin-induced redshift difference is most significant near the periapsis. The best strategy would be monitoring the star near its apoapsis and periapsis more frequently than at other locations in order to efficiently measure 

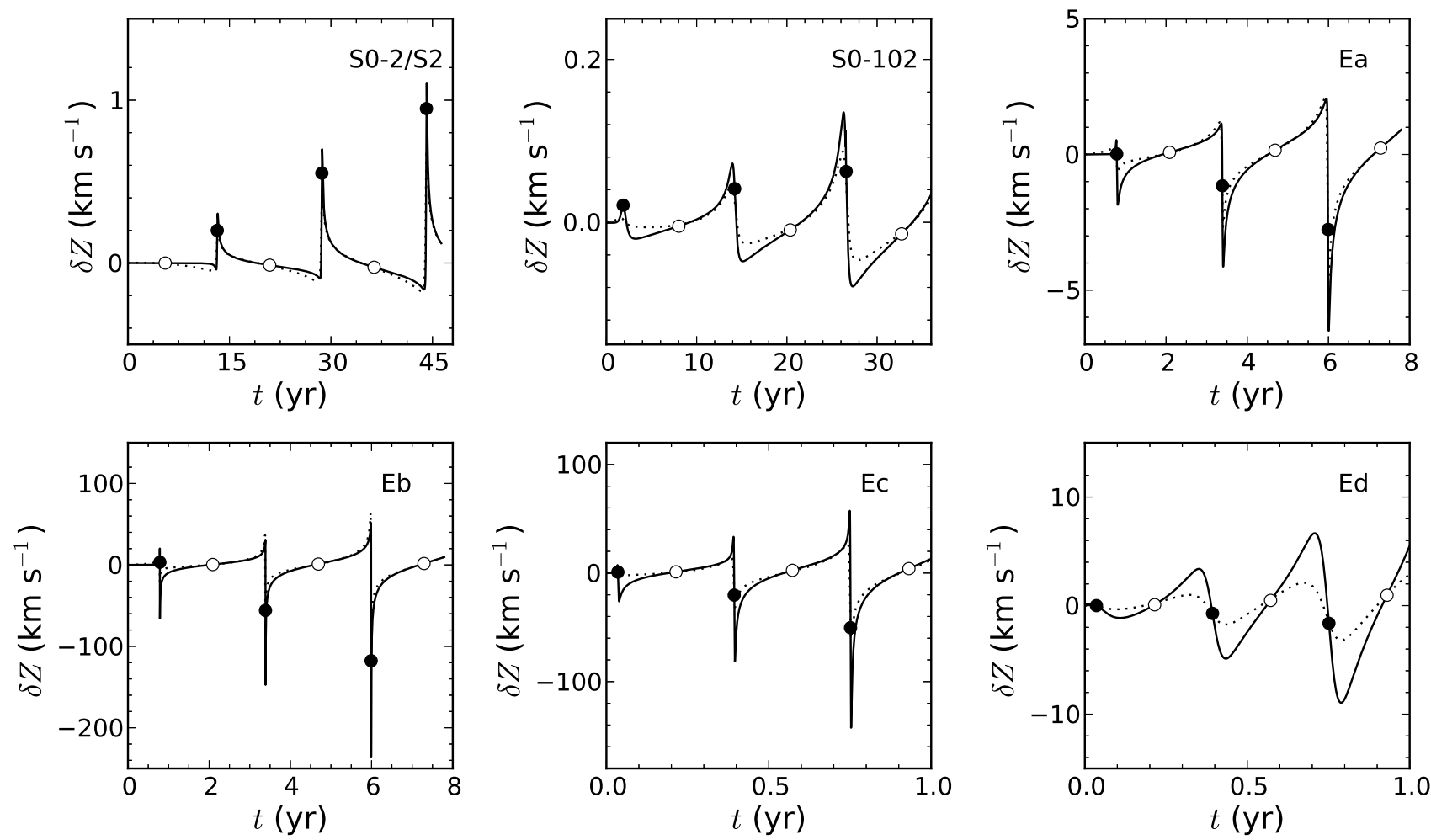

FIG. 10. - Difference between the redshift of each example star at each given moment for the case with a rapidly spinning MBH $(a=0.99)$ and that with a non-spinning MBH $(a=0)$. Panels from left to right, top to bottom, represent the example star S0-2/S2, S0-102, Ea, Eb, Ec, and Ed, respectively. Open and solid circles mark the locations of the apoapsides and periapsides, respectively. Solid lines and dashed lines represent the numerical results and analytical approximations obtained from Equation [36 respectively. Totally three orbits for each example star are shown here and the first orbit of each star starts at $t=0$.

the spin-induced effects and constrain the spin. Nevertheless, here we set the intervals between two consecutive observational times as $\delta t_{\mathrm{obs}} \propto r^{-1.5}$ (in the observer's rest frame). For each star, the total number of mock observations is 120 . Further assuming a set of expected accuracies on the apparent position $\left(\sigma_{\mathrm{p}}\right)$ and the redshift $\left(\sigma_{Z}\right)$ measurements of a star, e.g., $\left(\sigma_{\mathrm{p}}, \sigma_{Z}\right)=$ $\left(10 \mu \mathrm{as}, 1 \mathrm{~km} \mathrm{~s}^{-1}\right)$, we obtain the mock observations on the apparent position and the redshift of the star. The points in the top two panels of Figures 13 and 15 show the mock observations of the apparent positions (top left) and the redshifts (top right) for S0-2/S2 and Eb, respectively. With those mock data, we can use the following procedures to constrain the $\mathrm{MBH}$ properties (mass and spin) and the distance from the $\mathrm{MBH}$ to sun.

\subsection{MCMC Fitting Procedures}

1. For a given set of parameters for the $\mathrm{MBH}$ spin parameters $(a, i, \epsilon)$, the $\mathrm{MBH}$ mass $\left(M_{\bullet}\right)$, and the initial orbital elements of a model star $\left(a_{\mathrm{orb}, 0}, e_{\mathrm{orb}, 0}, \Omega_{0}^{\prime}, I_{0}^{\prime}, \Upsilon_{0}^{\prime}, v_{0}^{\prime}\right)$, the orbital motion of the star is generated over a period time that is the same as that of the mock one from full GR calculations as described in Sections 3. Note that the motion of the star is defined with respect to the central $\mathrm{MBH}$, which is approximated to be at rest in the Galactocentric rest frame. 6

6 The acceleration of the $\mathrm{MBH}$ with respect to the observer are negligible on the timescale considered in this paper $(\lesssim 50$ years).
2. According to the motion of the model star, we can obtain the apparent position $\left(\alpha_{j}, \beta_{j}\right)$ and the redshift $Z_{\mathrm{obs}, j}$ of the star at each observation time $t_{\mathrm{obs}, j}$, for any given distance of $R_{\mathrm{GC}}$ from the $\mathrm{MBH}$ to the Sun (see Section 4). In order to determine both the trajectory of the apparent positions of a

First, the acceleration of the Sun with respect to the GC is on the order of $10^{-6} \mathrm{~km} \mathrm{~s}^{-1}$ per year, and the total change of the relative velocity of the $\mathrm{MBH}$ due to this acceleration on the timescale $\lesssim 50$ years is on the order of $10^{-4} \mathrm{~km} \mathrm{~s}^{-1}$. Second, the Brownian motion of the $\mathrm{MBH}$ due to background stars is estimated to be on the order of $0.1 \mathrm{~km} \mathrm{~s}^{-1}$ and the change timescale may be larger than $10^{3}$ years (e.g., Merritt et al. 2007), so that the velocity change due to the Brownian motion should be $\lesssim 0.01 \mathrm{~km} \mathrm{~s}^{-1}$ on a timescale of 50 years. Therefore, the initial position of the $\mathrm{MBH}$ and the relative motion of the observer with respect to the $\mathrm{MBH}$ (to $\mathrm{Sgr} \mathrm{A}^{*}$ ) can be approximately described by the following six parameters: the distance from the $\mathrm{MBH}$ (or Sgr $\mathrm{A}^{*}$ ) to the observer, the initial position of the $\mathrm{MBH}$ ( or Sgr $\mathrm{A}^{*}$ ) on the sky plane (two parameters, and the proper motion and the radial motion of the observer with respect to the $\mathrm{MBH}$ (or $\mathrm{Sgr} \mathrm{A}^{*}$; three parameters). For an accurate reconstruction of the orbital configuration of a star, it is necessary to also include all of those parameters in the MCMC fitting procedures described below. For the purpose of demonstration purpose in the present paper, however, we assume that the MBH position is fixed (or the same as that of the $\operatorname{Sgr} \mathrm{A}^{*}$ ) on the sky plane and both the proper motion and the radial motion of the distant observer with respect to the $\mathrm{MBH}$ (or Sgr $\mathrm{A}^{*}$ ) are zero in the Galactocentric rest frame. Therefore, in the following MCMC fitting procedures, the $\mathrm{MBH}$ position is described by only one parameter, i.e., the distance from the distant observer to the $\mathrm{MBH}$ (or Sgr $\mathrm{A}^{*}$ ). With future high-quality observations (e.g., on the position and the motion of Sgr $\mathrm{A}^{*}$ ), it is possible to also constrain all of those six parameters, simultaneously, in practice. 


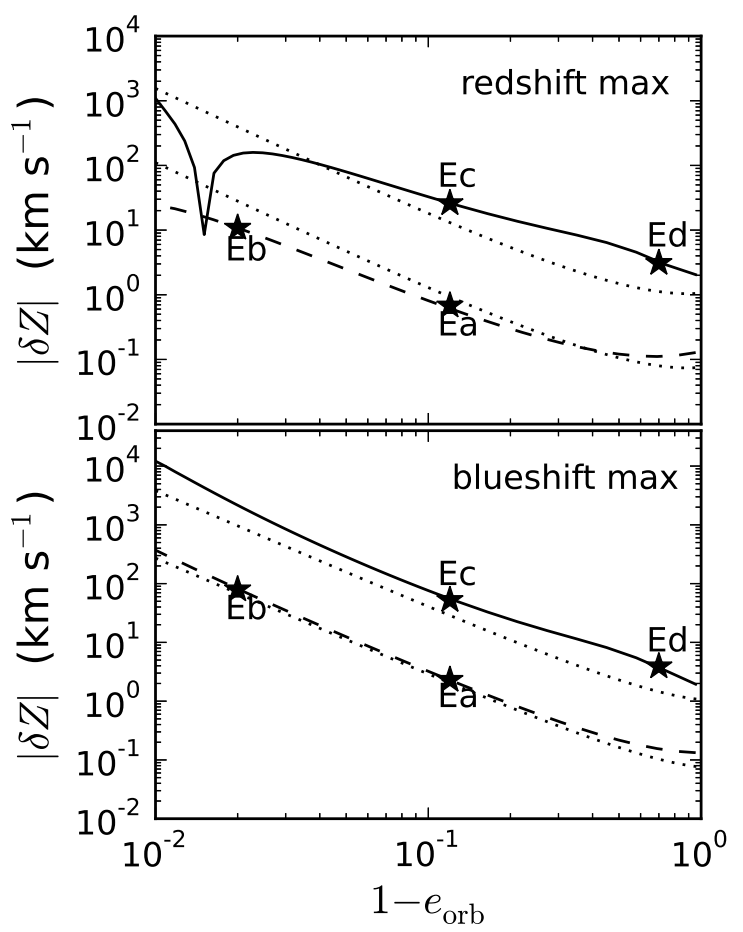

FIG. 11.- Maximum redshift difference (top panel) and the maximum blueshift difference (bottom panel) induced by the MBH spin. The MBH spin and its direction are assumed to be $a=0.99$ and $(i, \epsilon)=\left(45^{\circ}, 180^{\circ}\right)$. Solid and dashed lines represent the numerical results obtained in the same way as those shown in Fig. 10 for stars with the same orbital elements as those of Ec (solid lines) and Ea (dashed lines), respectively, except with various eccentricities. Dotted lines represent the estimates obtained approximately by Equation (36). This figure indicates that the spin-induced redshift is mainly contributed by the change of the orbital orientation due to the Lense-Thirring precession and the frame dragging.

star on the observer's sky plane and the redshift curve, totally 11 total parameters, i.e., $\Theta=$ $\left(R_{\mathrm{GC}}, M_{\bullet}, a, i, \epsilon, a_{\mathrm{orb}, 0}, e_{\mathrm{orb}, 0}, \Omega_{0}^{\prime}, I_{0}^{\prime}, \Upsilon_{0}^{\prime}, v_{0}^{\prime}\right)$, are needed. The exact values of the last six parameters for the initial orbital elements of the star are not interesting for the purpose of this study.

3 . To check whether the model star can fit the mock observations, we use the $\chi^{2}$-statistics for both the trajectory of the apparent position $\left(\alpha_{j}, \beta_{j}\right)$ and the redshift curve $\left(Z_{j}\right)$, i.e.,

$\chi_{\mathrm{p}}^{2}=\sum_{i=1}^{N}\left[\frac{\left(\alpha_{j}-\alpha_{\mathrm{obs}, j}\right)^{2}+\left(\beta_{j}-\beta_{\mathrm{obs}, j}\right)^{2}}{\sigma_{\mathrm{p}}^{2}}\right]$,

and

$$
\chi_{Z}^{2}=\sum_{j=1}^{N}\left[\frac{\left(Z_{j}-Z_{\mathrm{obs}, j}\right)^{2}}{\sigma_{Z}^{2}}\right],
$$

respectively, where $N$ is the total number of observations. If both the trajectory of the apparent positions and the redshift curve of the mock star are used for the fitting, we have $\chi^{2}=\chi_{\mathrm{p}}^{2}+\chi_{Z}^{2}$; however, if only one of the two data sets is used, we should have $\chi^{2}=\chi_{\mathrm{p}}^{2}$ or $\chi_{Z}^{2}$.

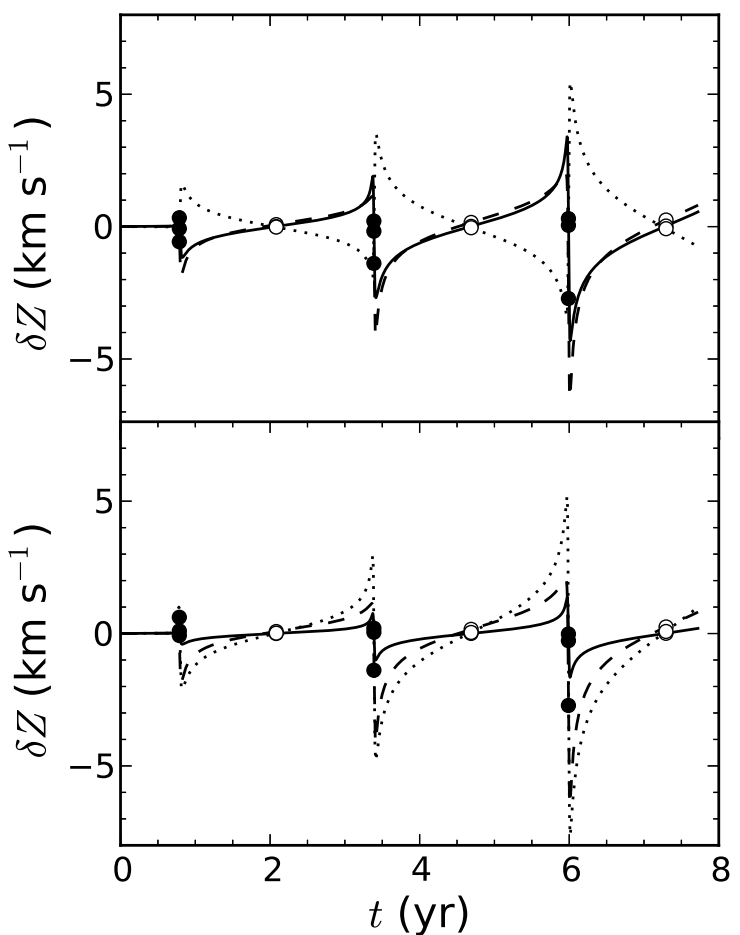

FIG. 12.- Dependence of the spin-induced redshift difference on the spin direction (top panel) and the star orbital orientation (bottom panel). Legend similar to Figure 7

4. For a given set of mock observations $\mathbf{D}$ of the apparent positions, the redshifts, or both, of a star, the posterior probability distributions of those parameters $\Theta$ can be constrained according to the Bayesian theorem, i.e., $P(\boldsymbol{\Theta} \mid \mathbf{D}) \propto P(\mathbf{D} \mid \boldsymbol{\Theta}) P(\boldsymbol{\Theta}) \propto$ $\exp \left(-\chi^{2} / 2\right) P(\boldsymbol{\Theta})$, where $P(\boldsymbol{\Theta})$ is the prior distribution of $\Theta$ and assumed to be flat over an initial guess range for each parameter. The MCMC method is adopted, with an implement of the Metropolis-Hasting algorithm, in order to efficiently get the best fit to the data $\mathbf{D}$ and obtain constraints on the model parameters $\Theta$ by searching the parameter space.

\subsection{Fitting Results}

Figure 13 illustrates the best fits to the mock observations of S0-2/S2, including both the apparent positions (solid line in the top-left panel) and redshifts (solid line in the top-right panel) The residuals of the fits are shown in the two middle panels. The solid circles in the two bottom panels show the difference between the mock observations and those predicted from a star with the same initial orbital elements around a non-spinning $\mathrm{MBH}$, and the solid lines represent the differences between the best fits and those predicted from the star around a non-spinning $\mathrm{MBH}$, similar to $\delta$ and $\delta Z$ shown in Figures 5 and 10, respectively. Obviously, the mock observations (points) are well fit by the model.

Figure 14] shows the two-dimensional contours and onedimensional probability distributions of the parameters $M_{\bullet}, R_{\mathrm{GC}}, a, i$, and $\epsilon$, respectively. The parameters of the best fit are listed in Table 2. As seen from Figure 14 and Table 2, the absolute value of the $\mathrm{MBH}$ spin can 
TABLE 2

Best-fit PARAmeters For the MBH mass AND SPIN AND the Distance to the GC.

\begin{tabular}{lccccc}
\hline Example Star & $a$ & $\begin{array}{c}i \\
\left({ }^{\circ}\right)\end{array}$ & $\begin{array}{c}\epsilon \\
\left({ }^{\circ}\right)\end{array}$ & $\begin{array}{c}\delta R_{\mathrm{GC}}{ }^{a} \\
(\mathrm{pc})\end{array}$ & $\begin{array}{c}\delta M_{\bullet}{ }^{b} \\
\left(10^{3} M_{\odot}\right)\end{array}$ \\
\hline S0-2/S2 & $0.812_{-0.257}^{+0.187}$ & $37_{-27}^{+39}$ & $156_{-89}^{+74}$ & $-0.30_{-0.97}^{+0.98}$ & $-0.41_{-1.15}^{+1.17}$ \\
S0-102 & $0.457_{-0.457}^{+0.542}$ & $92_{-82}^{+78}$ & $191_{-191}^{+169}$ & $-0.31_{-1.71}^{+1.69}$ & $0.52_{-2.47}^{+2.43}$ \\
Ea & $0.922_{-0.127}^{+0.077}$ & $51_{-13}^{+13}$ & $178_{-15}^{+14}$ & $-0.34_{-1.52}^{+1.39}$ & $-0.46_{-2.04}^{+1.93}$ \\
$\mathrm{~Eb}$ & $0.989_{-0.011}^{+0.010}$ & $45_{-1}^{+1}$ & $181_{-1}^{+1}$ & $-1.16_{-2.34}^{+2.50}$ & $-1.53_{-3.29}^{+3.55}$ \\
$\mathrm{Ec}$ & $0.987_{-0.015}^{+0.012}$ & $46_{-1}^{+2}$ & $178_{-6}^{+5}$ & $-1.07_{-2.61}^{+2.64}$ & $-0.80_{-3.21}^{+3.41}$ \\
$\mathrm{Ed}$ & $0.840_{-0.233}^{+0.159}$ & $61_{-23}^{+25}$ & $180_{-38}^{+35}$ & $-1.54_{-2.71}^{+2.69}$ & $1.12_{-2.79}^{+2.67}$ \\
\hline
\end{tabular}

Note. - Notes. The error associated with each parameter represents the 2- $\sigma$ error of the best-fit value of the parameter. ${ }^{a}$ The best fit of the distance to the GC is $R_{\mathrm{GC}}=8 \mathrm{kpc}+\delta \mathrm{R}_{\mathrm{GC}} .{ }^{b}$ The best fit of the MBH mass is $M_{\bullet}=4 \times 10^{6} M_{\odot}+\delta M_{\bullet}$.

be reasonably constrained by using the orbital motion of S0-2/S2 over two or three full orbits $(30-45$ years $)$, if (1) the astrometric and the redshift accuracies can reach $\sigma_{\mathrm{p}}=10 \mu$ as and $\sigma_{Z}=1 \mathrm{~km} \mathrm{~s}^{-1} ;(2)$ the $\mathrm{MBH}$ spin $a$ is close to one; and (3) the spin is pointing toward some directions with moderate or even maximum spin-induced effects on the trajectory of apparent position and the redshift curve, e.g., $(i, \epsilon)=\left(45^{\circ}, 200^{\circ}\right),\left(49^{\circ}, 126^{\circ}\right)$ or $\left(131^{\circ}, 306^{\circ}\right)$. According to Figures 5 and [10, $\delta$ mounts up with increasing number of orbits, therefore, the longer the time of monitoring the motion of a star, the tighter the constraints on the $\mathrm{MBH}$ spin obtained for a given set of $\left(\sigma_{\mathrm{p}}, \sigma_{Z}\right)$. If the astrometric and redshift accuracies can be a factor of a few times, or more, higher, i.e., $\sigma_{\mathrm{p}} \lesssim 3 \mu$ as and/or $\sigma_{Z} \lesssim 0.3 \mathrm{~km} \mathrm{~s}^{-1}$, the $\mathrm{MBH}$ spin can be constrained by using the motion of S0-2/S2 over only one full orbit (a period of $\sim 15$ year). However, if the astrometric and the redshift accuracies are a factor of two times or more lower, i.e., $\sigma_{\mathrm{p}}>20 \mu \mathrm{as}$ and/or $\sigma_{Z}>2 \mathrm{~km} \mathrm{~s}^{-1}$, then the MBH spin cannot be constrained by using the orbital motion of $\mathrm{S} 0-2 / \mathrm{S} 2$ within a few orbits.

Note here that both the distance to the GC and the $\mathrm{MBH}$ mass can be constrained simultaneously to an unprecedented high accuracy, i.e., $\delta M_{\bullet} / M_{\bullet} \sim$ a few times of $10^{-4}$ and $\delta R_{\mathrm{GC}} / R_{\mathrm{GC}} \sim$ a few times of $10^{-4}$. Even if the astrometric and redshift measurements are significantly less accurate, e.g., $\sigma_{\mathrm{p}}=50 \mu \mathrm{as}$ and/or $\sigma_{Z}=10 \mathrm{~km} \mathrm{~s}^{-1}$, the $\mathrm{MBH}$ mass and the distance to the $\mathrm{GC}$ can be still constrained to an accuracy of $0.98 \%$ and $0.36 \%$, respectively, although the MBH spin cannot be constrained, by monitoring the motion of S0-2/S2 over less than three full orbits. These results are consistent with those obtained by Weinberg et al. (2005).

If the absolute value of the $\mathrm{MBH}$ spin is smaller than 0.99 by a factor of $C$, then it requires the astrometric and/or redshift accuracies of $\sigma_{\mathrm{p}} \lesssim 10 C^{-1} \mu$ as and/or $\sigma_{Z} \lesssim C^{-1} \mathrm{~km} \mathrm{~s}^{-1}$ or a longer period for monitoring the star $(\gtrsim(2-3) C$ full orbits $)$, in order to distinguish the $\mathrm{MBH}$ from a Schwarzschild $\mathrm{MBH}$.

According to Figures 7 and [1], the amplitudes of $\delta$ and $\delta Z$ (due to the spin effects) also depend on the spin direction. However, the pattern of the dependence of $\delta$ on $(i, \epsilon)$ is somewhat different from that of $\delta Z$. For example, the amplitude of $\delta$ is the largest (or moderate) when $(i, \epsilon)=\left(72^{\circ}, 90^{\circ}\right)$ or $\left.\left(45^{\circ}, 180^{\circ}\right)\right]$; while the amplitude of $\delta Z$ is moderate but not the largest (or mod-
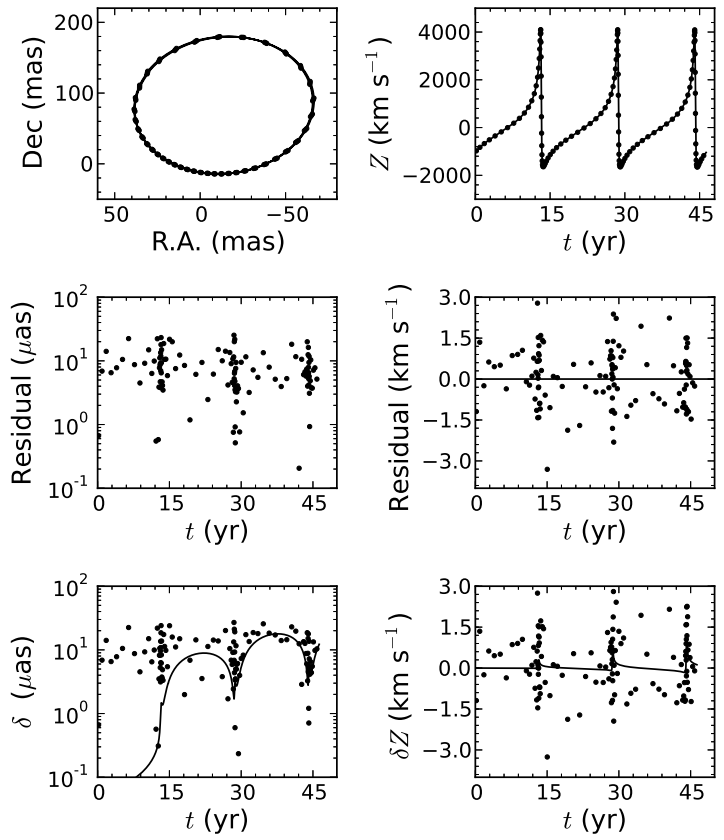

FIG. 13. - Mock trajectory of the apparent positions and the redshift curve of S0-2/S2 over three full orbital periods and their best fits. Top two panels are for the mock apparent positions (solid circles in the left panel) and the redshift curve (solid circles in the right panel) and their best fits (solid lines); middle panels are for the residuals of the best fits; and bottom panels represent the distances (solid circles in the left panel) or the redshift difference (solid circles in the right panel) between the mock observational positions (or redshifts) of S0-2/S2 and the positions (or redshifts) of an assumed star with the same initial orbital elements as the best fit of $\mathrm{S} 0-2 / \mathrm{S} 2$ but around a non-spinning $\mathrm{MBH}$, and the solid lines therein represent the differences between the predicted orbit of the best fit to S0-2/S2 and that of the assumed star.

erate but close to the largest) when $(i, \epsilon)=\left(72^{\circ}, 90^{\circ}\right)$ [or $\left.\left(159^{\circ}, 63^{\circ}\right)\right]$. If both measurements on redshifts and apparent positions with compatible accuracies are available, it would improve the constraint on the MBH spin and its direction. If only measurements on apparent positions with accuracy of $\sigma_{\mathrm{p}}=10 \mu$ as are available, then the spin is easier (or harder) to constrain by using the motion of S0-2/S2 within several orbits if $(i, \epsilon)=\left(72^{\circ}, 90^{\circ}\right)$ [or $\left.\left(159^{\circ}, 63^{\circ}\right)\right]$ (see more discussions in Yu et al. 2015).

Figure 15 illustrates the best fits to the mock observations of Eb, similar to that shown in Figure 13 for S0-2/S2. The two-dimensional contours and onedimensional probability distribution of the parameters 


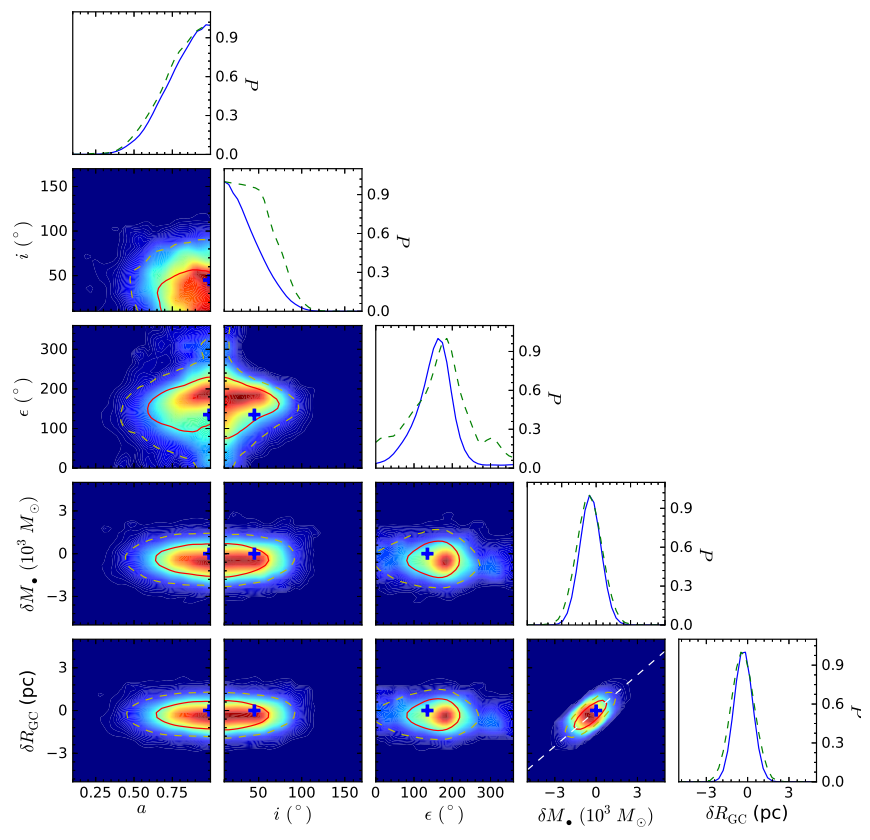

FIG. 14. - Two-dimensional probability contours and onedimensional probability distributions of the best-fit parameters for S0-2/S2. For the two-dimensional probability panels, the color contour maps represent the mean likelihood of the MCMC sample, and the line contours represent the marginalized distribution, with the red solid line and the yellow dashed line as the 1- $\sigma$ and $2-\sigma$ confidence level, respectively. The ' + ' symbol in each color map represents the original set of the parameter value to produce the mock observations. The white dashed line in the panel for $\delta R_{\mathrm{GC}}$ versus $\delta M_{\bullet}$ represents $M_{\bullet} \propto R_{\mathrm{GC}}^{\Gamma}\left(\right.$ and $\left.\delta M_{\bullet} / M_{\bullet}=\Gamma \delta R_{\mathrm{GC}} / R_{\mathrm{GC}}\right)$ with $\Gamma \sim 2.4$. For those panels showing the one-dimensional probability distributions, the blue solid line and the green dashed line represent the one-dimension marginalized distribution and the onedimensional mean likelihood, respectively.
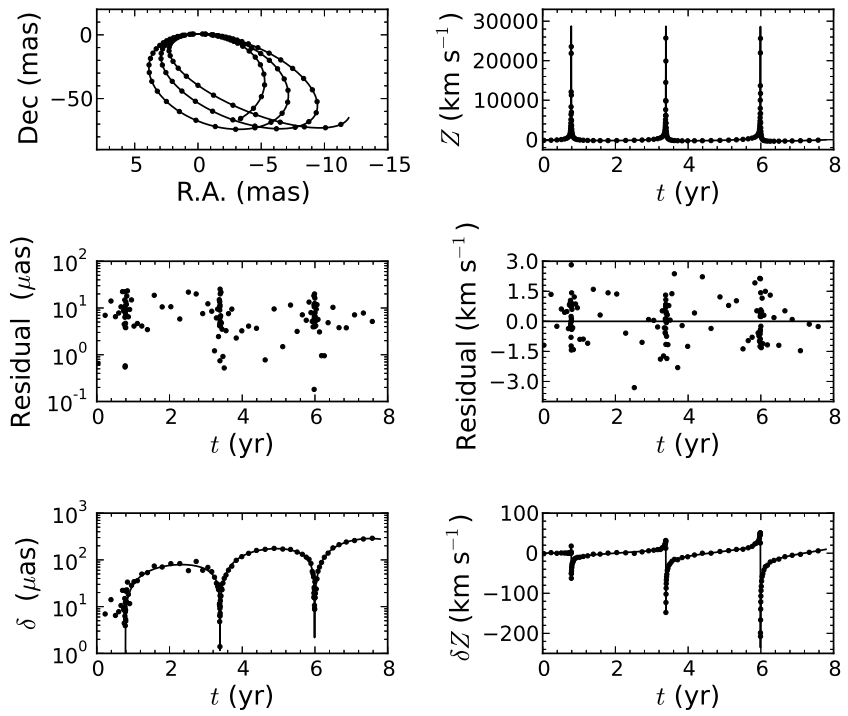

Fig. 15. - Legend similar to that for Fig. 13 but for the example star Eb.

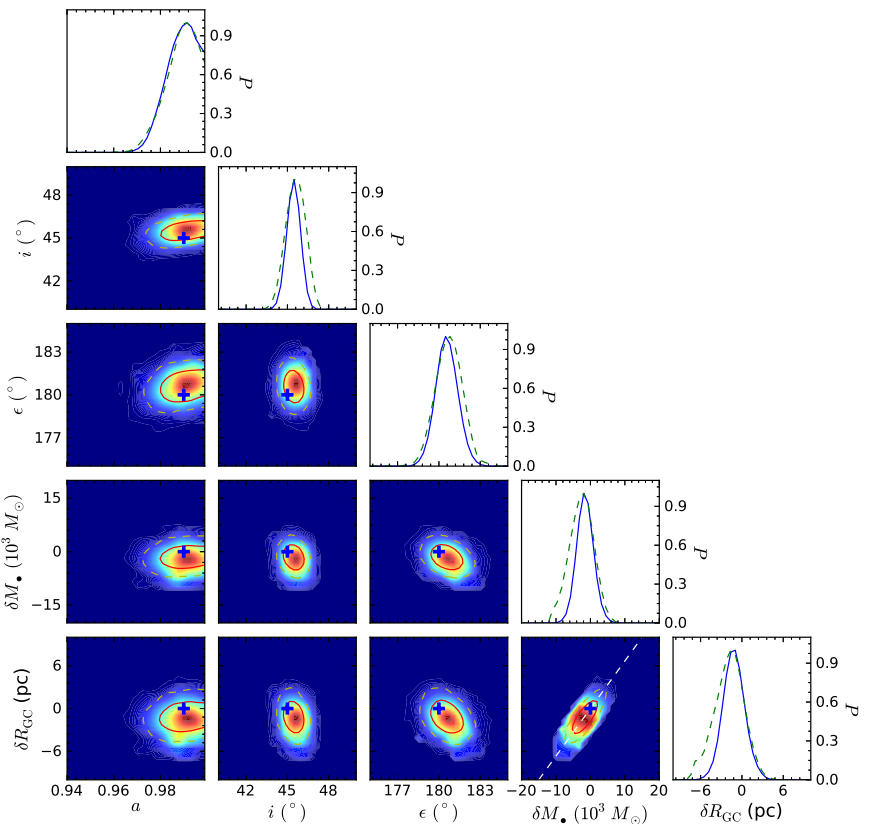

FIG. 16.- Legends similar to those for Fig. 14 but for the example star Eb. The dashed line in the panel for $\delta R_{\mathrm{GC}}$ versus $\delta M_{\bullet}$ represents $M_{\bullet} \propto R_{\mathrm{GC}}^{\Gamma}$ with $\Gamma \sim 3.0$.

are shown in Figure 16, and the best-fit parameters are listed in Table 2 Since Eb has a smaller semimajor axis (300 AU) and a larger eccentricity (0.98) compared with S0-2/S2, the spin-induced effects on $\delta$ and $\delta Z$ for Eb are larger than those for $\mathrm{S} 0-2 / \mathrm{S} 2$ by a factor of several tens to a hundred, respectively (see Figs. 5 and 10). Therefore, the $\mathrm{MBH}$ spin and its direction can be more accurately constrained by using the motion of Eb over three full orbits $(\sim 10$ years $)$ if $\left(\sigma_{\mathrm{p}}, \sigma_{Z}\right)=\left(10 \mu \mathrm{as}, 1 \mathrm{~km} \mathrm{~s}^{-1}\right)$, and the accuracy can be improved by more than one order of magnitude (see Tab. 2). For much lower astrometric and redshift precisions, e.g., $\sigma_{\mathrm{p}} \sim 50 \mu$ as and $\sigma_{Z} \sim 10 \mathrm{~km} \mathrm{~s}^{-1}$, which can be easily achieved by the next generation extremely large telescopes, it is still possible to constrain the $\mathrm{MBH}$ spin with considerable accuracy by using the motion of a star like Eb over a period less than a few full orbits ( $\lesssim 10$ years).

To produce the mock observations, we have assumed that the MBH spin $a=0.99$. If $a$ is set to be smaller than 0.99 by a factor of $D$, then the resulting amplitudes of $\delta$ and $\delta Z$ would be smaller than those shown in Figures 5 and 10 by the same factor (see eqs. B5, B8 and 36). If $D$ is less than 10 and $a \gtrsim 0.1$, we may still be able to constrain $a$ or distinguish it from a Schwarzschild BH by using the motion of a star like Eb within a few orbits provided $\left(\sigma_{\mathrm{p}}, \sigma_{Z}\right)=\left(10 \mu \mathrm{as}, 1 \mathrm{~km} \mathrm{~s}^{-1}\right)$. If $a \lesssim 0.5$ and $D \gtrsim 2$, however, it is difficult to get an accurate constraint on the spin value or distinguish it from a Schwarzschild $\mathrm{BH}$ by using the orbital motion of $\mathrm{S} 0-2 / \mathrm{S} 2$ over a few orbits, even if the spin vector is close to some specific direction [e.g., $\left(45^{\circ}, 200^{\circ}\right)$ or $\left(45^{\circ}, 135^{\circ}\right)$ ] with which the spin-induced effects on $\delta$ and/or $\delta Z$ are most significant. In this case, a constraint on whether the GC MBH is an extremely rotating $\mathrm{MBH}$ or not is possible. If the astrometric and redshift measurements are much more accurate (e.g., $\sigma_{\mathrm{p}} \lesssim 10 D^{-1} \mu$ as and /or $\sigma_{Z} \lesssim D^{-1} \mathrm{kms}^{-1}$ ) as 
discussed above, an accurate constraint on the MBH spin may still be obtained and it can be distinguished from a Schwarzschild BH.

Figure 17presents the one-dimensional probability distributions of the best-fit parameters for other stars, i.e., S0-102, Ea, Ec, and Ed, respectively. The best-fit parameters are listed in Table 2 As seen from Figure 17 and Table 2, the $\mathrm{MBH}$ spin cannot be constrained by using the relativistic orbital motion of S0-102 within a period of less than two to three orbits $(\lesssim 30-45$ years $)$ for $\sigma_{\mathrm{p}} \gtrsim 10 \mu$ as and $\sigma_{Z} \gtrsim 1 \mathrm{~km} \mathrm{~s}^{-1}$, even if the spin direction is close to the one with the largest effects on $\delta$ and $\delta Z$; it can be well constrained by using other stars, i.e., Ea, Ec, or Ed. The reason is that S0-102 has the largest pericenter distance and thus the least changes of $\delta$ and $\delta Z$ per orbit caused by the spin effects. The MBH spin is less well constrained by using Ed, compared with those using Ea and Ec, because the pericenter distance of Ed is larger, compared to Ea, Eb, and Ec, though its semimajor axis is smaller than that of Ea and Eb.

\section{DISCUSSIONS}

\subsection{Perturbations from Other Stars or Stellar Remnants}

In our full general relativistic calculations, the possible perturbation on the orbit and motion of a star due to other background stars existing within the star orbit is ignored. By assuming several different distributions of stars close to the GC MBH, Merritt et al. (2010) performed post-Newtonian simulations and showed that the stellar perturbation may obscure the signals due to frame dragging and quadruple moment for stars beyond $\sim 0.5-0.2 \mathrm{mpc}(\sim 100-400 \mathrm{AU})$; and Sadeghian \& Will (2011) also analytically investigated the perturbing effects of background stars and found that it may still be possible to detect relativistic precession of stars within a few tenth milliparsecs. Therefore, it may not be easy to use $\mathrm{S} 0-2 / \mathrm{S} 2$ (or even a star like $\mathrm{Ea}$ or $\mathrm{Eb}$ ) to constrain the GC MBH spin even if the accuracies of position and redshift measurements are sufficiently high (e.g., $\left.\sigma_{\mathrm{p}} \lesssim 10 \mu \mathrm{as}, \sigma_{Z} \lesssim 1 \mathrm{~km} \mathrm{~s}^{-1}\right)$. However, on the one hand, the exact number of stars or stellar remnants existing in the vicinity of the central $\mathrm{MBH}$ is not clear. It is not impossible that the stars or stellar remnants existing within the $\mathrm{S} 0-2 / \mathrm{S} 2$ (or $\mathrm{Ea} / \mathrm{Eb}$ ) orbit are very rare and their perturbations on the orbit and motion of S02/S2 (or Ea/Eb) are small and do not affect the spin signals much. Furthermore, the orbit-averaged torques from background stars are approximately constant in magnitude over year- to decade-long timescales, it is possible to disentangle the stellar perturbation effects from those due to the MBH spin as discussed in Merritt et al. (2010). On the other hand, it is an important step to first check whether the spin-induced effects on the motion of a star, as close to the GC MBH as those example stars, can be detected by ignoring the perturbations from other possibly existing stars within its orbit, with future facilities with sufficient astrometric and redshift accuracies.

Even if the perturbations from background stars on a target star are not negligible, the observational data collected around the pericenter, where the perturbations are substantially less significant than those near the apocenter, may still be useful to constrain the $\mathrm{MBH}$ spin pa- rameters (see Angélil \& Saha 2014). By using the redshift data near the pericenter, strong constraints on spin parameters may be obtained since the spin-induced redshift differences are the most significant near the pericenter. However, the constraints on spin parameters may be much weaker if only the position data are used because the spin-induced position displacements near the pericenter are substantially smaller than those near the apocenter. As a check, we perform additional MCMC fittings for $\mathrm{S} 2 / \mathrm{S} 0-2$ and $\mathrm{Eb}$ by only using the mock observations near the pericenter (i.e., $r<a_{\text {orb }}$ or $r \ll a_{\text {orb }}$ ). Our results show that the $\mathrm{MBH}$ spin parameters are poorly constrained by using the mock observations near the pericenter of S2/S0-2 $\left(0 \lesssim a \lesssim 1, i=61^{\circ}{ }_{-61^{\circ}}\right.$, and $\epsilon=160^{\circ}{ }_{-113^{\circ}}$ ) because the spin-induced maximum redshift difference can only be marginally detected (see Figure 10). For Eb, however, the spin parameters can be well constrained $\left(a=0.99_{-0.012}^{+0.009}, i=45_{-1^{\circ}}^{\circ}\right.$, and $\left.\epsilon=182_{-3^{\circ}}^{\circ}\right)$ because the spin-induced redshift differences near the pericenter are substantially higher than the detection limit and can be accurately measured.

It is also possible that an intermediate mass black hole (IMBH) exists in the vicinity of the GC MBH (e.g., Yu \& Tremaine 2003; Hansen \& Milosavljevic 2003; Genzel et al. 2010), and this IMBH may perturb the orbit and motion of a star near the MBH. Gualandris et al. (2010) have suggested that future observations on the S0-2/S2 orbit may be used to probe or constrain the existence of an IMBH in the GC.

We also note here that other effects, such as those from gas dynamics, wind mass loss, and tidal dissipation, may affect the orbit and motion of a star close to the $\mathrm{MBH}$. Psaltis (2012) and Psaltis et al. (2013) have shown that these effects may be negligible and do not preclude the measurements of the MBH spin and quadruple moment.

\subsection{Accurately Constraining the MBH Mass and the Distance from the Sun to the $G C$}

With the expected astrometric and redshift accuracies of the next generation facilities $\left[\left(\sigma_{\mathrm{p}}, \sigma_{Z}\right)=\right.$ $\left(10 \mu \mathrm{as}, 1 \mathrm{kms}^{-1}\right)$ or $\left.\left(50 \mu \mathrm{as}, 5 \mathrm{~km} \mathrm{~s}^{-1}\right)\right]$, the $\mathrm{MBH}$ mass and the distance from the Sun to the GC can be constrained with an unprecedented accuracy on the order of a few $0.01 \%$ to $0.1 \%$ or a few $0.1 \%$ to $1 \%$ if using S0-2/S2 (see early works by Salim \& Gould 1999; Weinberg et al. 2005). Even with such high accuracies of $\sigma_{\mathrm{p}}$ and $\sigma_{Z}$, the degeneracy between the $\mathrm{MBH}$ mass and the distance from the Sun to the GC remains, i.e., $M_{\bullet} \propto R_{\mathrm{GC}}^{\Gamma}$ $\left(\delta M_{\bullet} / M_{\bullet}=\Gamma \delta R_{\mathrm{GC}} / R_{\mathrm{GC}}\right)$ and $\Gamma \sim 2.4-3.0$ (e.g., see Figures 14 and 16), similar to that found in Ghez et al. (2008, see Fig. 11 therein) and Gillessen et al. (2009, see Fig. 15 therein). Although the $\mathrm{MBH}$ spin can be better constrained by using the orbital motion of a star with a small semimajor axis and a high eccentricity, such as $\mathrm{Eb}$, the constraints on $M_{\bullet}$ and $R_{\mathrm{GC}}$ are not significantly improved. The tightness of the constraints on $M_{\bullet}$ and $R_{\mathrm{GC}}$ depends on the ratio of the astrometric accuracy to the semimajor axis and the ratio of the redshift accuracy to the orbital velocity, but does not depend directly on the semimajor axis and eccentricity of the test star. 

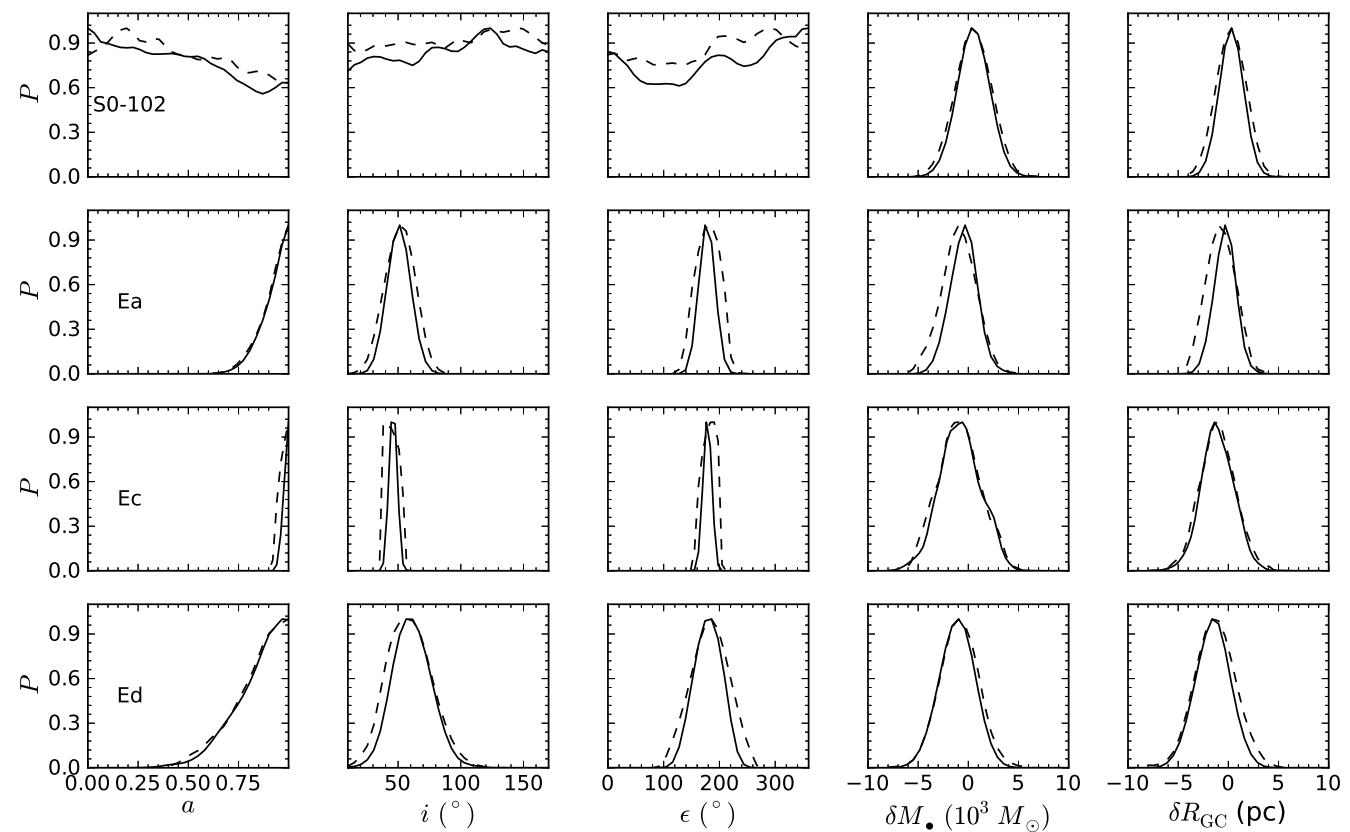

FIG. 17.- One-dimensional probability distributions of the best-fit parameters for other stars obtained in the same way as done in Figs. 14 and 16. Top to bottom rows are for S0-102, Ea, Ec, and Ed, respectively. The solid line and the dashed line represent the one-dimension marginalized distribution and the one-dimensional mean likelihood, respectively.

\subsection{Usefulness of the Observations of Stars with Very High Eccentricities during Their Pericentric Passages}

In the GC, some stars may move around the $\mathrm{MBH}$ on nearly parabolic orbits $\left(e_{\mathrm{orb}}>0.95\right)$ as suggested by the existence of the star S0-16/S14 (with $e_{\text {orb }}=0.963$ and pericenter distance of $\sim 70 \mathrm{AU}$; Gillessen et al. 2009). With such high eccentricities, they may approach the MBH at a distance even substantially smaller than those stars with smaller semimajor axes, such as S0-2/S2, and thus more significantly affected by the spin effects. As demonstrated in Section 5, the spin-induced position displacements and redshift differences are most significant near the apoapsis and periapsis, respectively. Since the orbital periods of those stars on nearly parabolic orbits may be substantially longer than those with smaller semimajor axes (e.g., S0-2/S2 and S0-102), it is difficult to monitor them for a whole orbit. However, it should be useful to monitor the motion of those stars during their pericentric passage, when the spin-induced redshift differences (but not the position displacements) are most significant. We further check the validity of using the observations near the pericentric passage of a star on nearly parabolic orbit and find that the $\mathrm{MBH}$ may be accurately constrained if the pericenter distance of the star is smaller than a few tens AU. If there are more than one such stars, which would pass their periapsides, in the near future, monitoring their motions would be helpful in constraining the MBH spin.

\subsection{Direction of the MBH Spin}

The spin-induced GR effects, e.g., the Lense-Thirring precession and the frame dragging, on the orbit of a star depend on the direction of the spin. For a star with a given orbital orientation, the spin-induced effects on the changes of its apparent positions (or redshift) may vary by a factor of several to more than an order of magnitude if choosing significantly different spin directions, and the spin-induced effects are negligible for some spin directions, even if the absolute value of the spin is large and the semimajor axis of the star is small. However, the patterns of the changes of the position displacements and those of the redshift differences are different (see Figures 7 and 12). Therefore, simultaneously using both the trajectory of the apparent positions and the redshift curve would help to obtain a better constraint on the spin, provided that the maximum values of the spininduced position displacements and redshift differences over the observing period are substantially larger than the astrometric and redshift accuracy, respectively (see discussions in $\mathrm{Yu}$ et al. 2015). In this case, if only choosing one of the two data sets, the constraint on the spin would become losser because of the above orientation dependence. If the relativistic orbital motions of more than one star with significant different orbital orientations are available, the constraints on the $\mathrm{MBH}$ spin would also be tightened.

\subsection{Test for Alternative Metrics}

In this study, the metric of the massive object (presumably an $\mathrm{MBH}$ ) in the GC is assumed to be described by a Kerr metric. In principle, this object could be described by a metric, different from the Kerr metric (see Will 1993), e.g., the Johannsen-Psaltis metric (Johannsen \& Psaltis 2011). Similarly, it is also possible to use the relativistic orbits of stars in the immediate vicinity of the central object to check whether the central object is described by such alternative metrics or not. To do this, a modification to the numerical calculations of the orbits and the ray tracing in such a metric is required, which is deferred to a future study.

\section{CONCLUSIONS}


The innermost S-star discovered in the vicinity of the $\mathrm{GC} \mathrm{MBH}$ is believed to be able to provide strong constraints on the MBH spin and metric, and thus provide a strong test to the no-hair theorem of BHs and GR. In this paper, we develop a full general relativistic method to simultaneously constrain the MBH mass, spin, and its direction, and the distance from the Sun to the GC, by using the relativistic orbital motion of a star close to the $\mathrm{MBH}$.

The spin information is encoded in the trajectory of the apparent position of the star in the observer's sky plane and the redshift curve measured by the observer, which include: (1) the changes in the orbital motion induced by the MBH spin and (2) the changes of the photon trajectory propagating from the star to a distant observer due to the spin. We numerically solve the motion equations in the Kerr metric for a star rotating around an $\mathrm{MBH}$; and we follow the propagation of photons from the star to the distant observer by using a full general relativistic raytracing technique. Armed with these numerical methods, we can obtain the trajectory of the apparent position and the redshift curve of any star, with any given initial conditions, rotating around an $\mathrm{MBH}$, with any given mass and spin (and the spin direction). We adopt six example stars with different semi-major axes and eccentricities. Two of these stars have orbital configurations similar to that of S0-2/S2 and S0-102, respectively; and the other four are adopted according to the probability distribution of the orbit of a star that is expected to be the closest one to the GC MBH. We obtain the trajectories of the apparent positions and redshift curves for those stars over a given period, and further obtain the mock observations of both the trajectories of their apparent position and their redshift curves by assuming astrometric $\left(\sigma_{\mathrm{p}}\right)$ and redshift accuracies $\left(\sigma_{Z}\right)$ of future facilities. According to our calculations, we emphasize some points below.

First, the spin-induced effects on the orbital motion depend on both the absolute spin value $(a)$ and spin direction. The amplitude of the spin-induced position displacements and redshift differences can differ significantly (by an order of magnitude) for MBHs with different spin directions but the same absolute spin value.

Second, for $\mathrm{S} 0-2 / \mathrm{S} 2$, the spin-induced effects would lead to a maximum change of $13 \mu \mathrm{as}$ in the apparent position of the star within a full orbit for a maximum spin with direction of $\left(49^{\circ}, 125^{\circ}\right)$ [or $\left.\left(131^{\circ}, 305^{\circ}\right)\right]$. These values are consistent with previous estimates (e.g., Will 2008; Angélil et al. 2010). The maximum change depends on the spin direction. The changes in the apparent positions are mainly due to the spin-induced changes of the orbital motion, while the part due to the spin-induced changes of the photon propagation path is on the order of $0.01 \mu \mathrm{as}$, only $\sim 0.1 \%$ of the total changes.

Third, for S0-2/S2, the spin-induced effects would also lead to a maximum change of the redshifts $\left(\sim 0.3 \mathrm{~km} \mathrm{~s}^{-1}\right)$ over a full orbit for a maximum spin with direction of $\left.\left(129^{\circ}, 171^{\circ}\right)\right]$ or $\left.\left(51^{\circ}, 351^{\circ}\right)\right]$. The dependence of the maximum changes in the redshift curves on the spin direction is different from that of the maximum changes in the apparent positions. The spin-induced maximum change of the redshift over a full orbit is also dominated by the orbital change, and the contribution from the photon propagation is on the order of $0.003 \mathrm{~km} \mathrm{~s}^{-1}$, about the percentage level of the total changes.

Fourth, for a star much closer to the MBH than S0$2 / \mathrm{S} 2$, such as Eb with a semimajor axis of $\sim 300 \mathrm{AU}$ and an eccentricity of $\gtrsim 0.98$, the spin-induced effects would lead to a maximum change of $308 \mu$ as in its apparent position within a full orbit for a maximum spin with a direction of $\sim\left(111^{\circ}, 275^{\circ}\right)$ [or $\left.\sim\left(69^{\circ}, 95^{\circ}\right)\right]$; and it would also lead to a maximum change of $\sim 93 \mathrm{~km} \mathrm{~s}^{-1}$ in the redshifts over a full orbit for a maximum spin with direction of $\sim\left(150^{\circ}, 319^{\circ}\right)$ [or $\left.\sim\left(30^{\circ}, 139^{\circ}\right)\right]$. The spin signals of a star like Eb can be more than an order of magnitude higher than those of S0-2/S2, and thus monitoring the motion of such a star could provide much tighter constraints on the $\mathrm{MBH}$ spin and metric. The maximum value of the spin-induced redshift differences over a full orbit is still dominated by the orbital change, and the contribution from the photon propagation is on the level of $10 \%$ of the total changes.

Finally, in order to accurately constrain the spin and metric of the GC MBH (on the percentage level), it is necessary to consider both the spin effects on the stellar orbit and the photon propagation from the star to the distant observer. Although the changes in the apparent position of a star due to the spin effects on photon propagations are only about $0.001 \%-0.1 \%$ of those caused by the change of the orbital motion, the changes in the redshift of a star due to the spin effect can be up to $\sim 1 \%-10 \%$ of those caused by the change of the orbital motion if the semimajor axis is in the range from 1000 to $100 \mathrm{AU}$. Therefore, it is important to include the full GR calculations of the photon propagations in order to accurately constrain the $\mathrm{MBH}$ spin.

Adopting the MCMC method, we illustrate that the spin of the GC MBH may be able to be constrained by using the orbital motion of S0-2/S2 over a period of $\sim 45$ years if (1) the spin value $a$ is close to 1 , (2) $\sigma_{\mathrm{p}} \sim 10 \mu \mathrm{as}$ and $\sigma_{Z} \sim 1 \mathrm{~km} \mathrm{~s}^{-1}$, which will be achieved by the new instrument GRAVITY on VLTI, and (3) the spin is pointing toward some specific direction, with which the spininduced effects are likely moderate to most significant. If the astrometric and spectroscopic precisions can be further improved by a factor of several, then the GC $\mathrm{MBH}$ spin may be well constrained by using the relativistic motion of S0-2/S2 monitored over a period of no more than 15 years. In the mean time, the distance from the Sun to the GC and the MBH mass can also be constrained to an unprecedented accuracy $(0.01-0.1 \%)$. If there exists a star with a semimajor axis of less than several hundred AU and an eccentricity of $\gtrsim 0.9$ as expected (see Zhang et al. 2013), the MBH spin can be constrained with high accuracy over a period of $\sim 15$ years for $\sigma_{\mathrm{p}} \sim 10 \mu \mathrm{as}$ and $\sigma_{Z} \sim 1 \mathrm{~km} \mathrm{~s}^{-1}$, even if $a$ is only moderately large. The next generation facilities, such as the GRAVITY on VLTI, TMT, and E-ELT, may reach an astrometric accuracy of $10-50 \mu \mathrm{as} 7$ Those extremely large telescopes may reveal some stars, if existing, with semimajor axes less than a few hundred AU and eccentricities $\gtrsim 0.9$, within the S0-2/S2 (or S0-102) orbit. The spin (and its direction) of the $\mathrm{GC} \mathrm{MBH}$ is likely to be tightly constrained by using the relativistic motions of such stars. Long time monitoring of the orbital motions

7 http://www.tmt.org; http://www.eso.org/sci/facilities/eelt/ 
of stars in the vicinity of the GC MBH by the next generation facilities is likely to provide an ultimate dynamical test for the first time to the no-hair theorem and GR.

We thank Scott Tremaine for bringing our attention to the work by A. Angélil and P. Saha. This work was supported in part by the National Natural Science Foundation of China under grant Nos. 11273004, 11373031, and 11390372, the Strategic Priority Research Program "The Emergence of Cosmological Structures" of the Chinese Academy of Sciences, grant No. XDB09000000, the Bairen program from the National Astronomical Observatories of China, Chinese Academy of Sciences, and a grant from the John Templeton Foundation and National Astronomical Observatories of Chinese Academy of Sciences. F.Z. is supported by a postdoctoral fund $2014 \mathrm{M} 550549$.

\section{REFERENCES}

Abramowitz, M., \& Stegun, I. A. 1972, Handbook of Mathematical Functions (New Yorker: Dover)

Angélil, R., \& Saha, P. 2010, ApJ, 711, 157

Angélil, R., Saha, P., \& Merritt, D. 2010, ApJ, 720, 1303

Angélil, R., \& Saha, P. 2011, ApJL, 734, 19

Angélil, R., \& Saha, P. 2014, MNRAS, 444, 3780

Bardeen, J. M., Press, W. H., \& Teukolsky, S. A. 1972, ApJ, 178, 347

Bardeen, J. M. 1973, in Black holes, ed. C. DeWitt \& B. S., DeWitt (New York: Gordon \& Breach), 215

Barker, B. M., \& O'Connell, R. F. 1975, Phys. Rev. D, 12, 329

Boyer, R. H., \& Lindquist, R. W. 1967, Journal of Mathematical Physics, 8, 265

Byrd, P. F., \& Friedman, M. D. 1954, Handbook of Elliptic Integrals for Engineers and Physicists (Berlin: Spinger)

Čadež, A., Fanton, C., \& Calvani, M. 1998, New Astronomy, 3, 647

Chandrasekhar, S. 1983, The Mathematical Theory of Black holes (Oxford: Oxford Univ. Press)

Cunningham, C. T., \& Bardeen, J. M. 1973, ApJ, 183, 237

Dexter, J., \& Agol, E. 2009, ApJ, 696, 1616

Doeleman, S. S., Fish V. L., Broderick, A. E., Loeb, A., \& Rogers, A. E. E. 2009, ApJ, 695, 59

Dormand, J. R., \& Prince, P. J. 1980, J. Comp. Appl. Math., Vol.6, p.19

Fragile, P. C., \& Mathews, G. J. 2000, ApJ, 542, 328

Genzel, R., Eisenhauer, F., \& Gillessen, S. 2010, Reviews of Modern Physics, 82, 3121

Ghez, A., Salim, S., Weinberg, N. N., Lu, J. R., Do, T., Dunn, J. K., Matthews, K., Morris, M. R., Yelda, S., Becklin, E. E., et al. 2008, ApJ, 689, 1044

Gillessen, S., Eisenhauer, F., Trippe, S., Alexander, T., Genzel, R., Martins, F., \& Ott, T. 2009, ApJ, 692, 1075

Gualandris, A., Gillessen, S., \& Merritt, D. 2010, MNRAS, 409, 1146

Hairer, E., Norsett, S. P., \& Wanner, G. 1987, Solving Ordinary Differential Equations I. Nonstiff Problems, Springer Series in Comput. Mathematics, Vol. 8 (Springer-Verlag)

Hansen, B. M. S., \& Milosavljević, M. 2003, ApJ, 593, L77

Iorio, L. 2011a, Phys. Rev. D, 84, 124001

Iorio, L. 2011b, MNRAS, 411, 453

Jaroszynski, M. 1998, Acta Astron., 48, 653

Johannsen, T., \& Psaltis, D. 2011, Phys. Rev. D, 83, 124015

Kannan, R., \& Saha, P. 2009, ApJ, 690, 1553

Karas, V., Vokrouhlicky, D., \& Polnarev, A. G. 1992, MNRAS, 259,569

Kerr, R. P. 1963, Physical Review Letters, 11, 237

Kopeikin, S. M., \& Makarov, V. V. 2007, Phys. Rev. D, 75, 062002
Kormendy, J., \& Ho, L. C. 2013, ARA\&A, 51, 511

Li, L.-X., Zimmerman, E. R., Narayan, R., \& McClintock, J. E. 2005, ApJS, 157, 335

Lu, Y., \& Yu, Q. 2001, ApJ, 561, 660

Magorrian, J., Tremaine, S., Richstone, D., et al. 1998, AJ, 115, 2285

Magorrian, J., \& Tremaine, S. 1999, MNRAS, 309, 447

McClintock, J. E., Narayan, R., Davis, S. W., et al. 2011, CQGra, 28,114009

Merritt, D., Berczik, P., \& Laun, F. 2007, AJ, 133, 553

Merritt, D., Alexander, T., Mikkola, S., \& Will, C. M. 2010, Phys. Rev. D, 81, 062002

Meyer, L., Ghez, A. M., Schödel, R., et al. 2012, Science, 338, 84

Misner, C. W., Thorne, K. S., \& Wheeler, J. A. 1973, Gravitation (San Francisco: W. H. Freeman and Co.)

Narayan, R., \& McClintock, J. E. 2013, arXiv:1312.6698

Peters, P. C. 1964, Phy. Rev., 136, 1224

Preto, M., \& Saha, P. 2009, ApJ, 703, 1743

Psaltis, D. 2012, ApJ, 759, 130

Psaltis, D., Li, G., \& Loeb, A. 2013, ApJ, 777, 57

Rauch K. P., \& Blandford R. D. 1994, ApJ, 421, 46

Reynolds, C. S., 2013, CQGra, 30, 244004

Rubilar, G. F., \& Eckart, A. 2001, A\&A, 374, 95

Sadeghian, L., \& Will, C. M. 2011, Classical and Quantum Gravity, 28, 225029

Salim, S., \& Gould, A. 1999, ApJ, 523, 633

Schödel, R., Ott, T., Genzel, R., et al. 2002, Nature, 419, 694

Thorne, K. S. 1986, "Black Holes: The Membrane Viewpoint," in Highlights of Modern Astrophysics: Concepts and

Controversies, eds. Stuart L. Shapiro and Saul A. Teukolsky (John Wiley \& Sons, New York, 1986), pp. 103-161

Weinberg, N. N., Milosavljević, M., \& Ghez, A. M. 2005, ApJ, 622,878

Wex, N., \& Kopeikin, S. M. 1999, ApJ, 514, 388

Will, C. M. 1993, Theory and Experiment in Gravitational Physics (Cambridge: Cambridge Univ. Press)

Will, C. M. 2008, ApJ, 674, L25

Yu, Q., \& Lu, Y. 2000, MNRAS, 311, 161

Yu, Q., \& Tremaine, S. 2003, ApJ, 599, 1129

Yu, Q., Zhang, F., \& Lu, Y. 2015, submitted

Zhang, F., Lu, Y., \& Yu, Q. 2013, ApJ, 768, 153

Zhang, F., Lu, Y., \& Yu, Q. 2014, ApJ, 784, 106

Zucker, S., Alexander, T., Gillessen, S., Eisenhauer, F., \& Genzel, R. 2006, ApJ, 639, L21

\section{APPENDIX}

\section{A. PHOTON ORBITS IN A KERR SPACETIME}

The motion of a photon in a Kerr spacetime is given by Equations (3)-(6). We assume that an observer, located at $\left(r_{0}, \theta_{0}, \phi_{0}\right)=\left(10^{8} r_{\mathrm{g}}, i, 0\right)$ in the BH rest frame, receives photons emitted from a star located at $\overrightarrow{\mathbf{R}}_{\star}=\left(r_{\star}, \theta_{\star}, \phi_{\star}\right)$, which is rotating around the BH. Define $\mu=\cos \theta$, the motions of those photons in the $r-\theta$ plane are given by (Bardeen et al. 1972; Cunningham \& Bardeen 1973; Chandrasekhar 1983)

$$
\int_{r_{0}}^{r_{\mathrm{hit}}} \frac{d r}{\sqrt{R}}= \pm \int_{\mu_{0}}^{\mu_{\star}} \frac{d \mu}{\sqrt{\Theta_{\mu}}},
$$


where $\mu_{0}=\cos i, \mu_{\star}=\cos \theta_{\star}$, and $r_{\text {hit }} \rightarrow r_{\star}$ is the redshift of a photon when $\mu=\mu_{\star}$, i.e., the photon hits the surface of the star at $\left(r_{\text {hit }}, \theta_{\star}\right)$. In Equation (A1), the left side is integrated from a distant observer to the surface of the star and it should be negative as $r_{0}>r_{\text {hit }}$. We should carefully choose the sign in front of the integration at the right side, i.e., if $\mu_{0}<\mu_{\star}$, the sign should be "-"; and if $\mu_{0}>\mu_{\star}$, the sign should be "+". Below, we denote the left and right side of Equation (A1) as $\mathscr{I}_{\mu}$ and $\mathscr{J}_{r}$, respectively.

\section{A.1. Integral over the $\theta$-direction}

We denote the integral over $\tilde{\mu}$ on the left side of Equation (A1) as

$$
\mathscr{F}_{\mu}(\mu)=\int \frac{d \tilde{\mu}}{\sqrt{\Theta(\tilde{\mu})}}
$$

where

$$
\begin{gathered}
\Theta(\tilde{\mu})=q^{2}+\left(a^{2}-q^{2}-\lambda^{2}\right) \tilde{\mu}^{2}-a^{2} \tilde{\mu}^{4}=a^{2}\left(\mu_{-}^{2}+\tilde{\mu}^{2}\right)\left(\mu_{+}^{2}-\tilde{\mu}^{2}\right), \\
\mu_{ \pm}^{2}=\frac{1}{2 a^{2}}\left[\sqrt{\left(\lambda^{2}+q^{2}-a^{2}\right)^{2}+4 a^{2} q^{2}} \mp\left(\lambda^{2}+q^{2}-a^{2}\right)\right]
\end{gathered}
$$

and $-\mu_{+}$and $\mu_{+}$are the two real roots of $\Theta(\tilde{\mu})=0$. Because $\Theta(\tilde{\mu})$ must be $\geq 0$, the physical allowed region for a photon is $-\mu_{+} \leq \tilde{\mu} \leq \mu_{+}$. Once $\tilde{\mu}$ reaches the turning point (either $\mu_{+}$or $-\mu_{+}$) and bouncing back, the $\tilde{\mu}$-integral at the right side of Equation (A5) changes the sign. If the integration range of the $\theta$-integral is from $\mu(\geq 0)$ to $\mu_{+}$, we may denote the function $\mathscr{F}_{\mu}(\mu)$ explicitly as

$$
\mathscr{F}_{\mu}(\mu)=\int_{\mu}^{\mu_{+}} \frac{d \tilde{\mu}}{\sqrt{\Theta(\tilde{\mu})}}
$$

If $-\mu_{+} \leq \mu<0$, we have

$$
\int_{\mu}^{0} \frac{d \tilde{\mu}}{\sqrt{\Theta(\tilde{\mu})}}=\int_{0}^{\mu_{+}} \frac{d \tilde{\mu}}{\sqrt{\Theta(\tilde{\mu})}}-\int_{-\mu}^{\mu_{+}} \frac{d \tilde{\mu}}{\sqrt{\Theta(\tilde{\mu})}}=\mathscr{F}_{\mu}(0)-\mathscr{F}_{\mu}(-\mu)
$$

According to Byrd \& Friedman (1954, p48, eq. 213.00), $\mathscr{F}_{\mu}(\mu)$ can be expressed in terms of an inverse Jocabian elliptic function

$$
\mathscr{F}_{\mu}(\mu)=g_{\mu} \mathrm{cn}^{-1}\left(\cos \varphi \mid m_{\mu}\right)=g_{\mu} F\left(\varphi \mid m_{\mu}\right)
$$

where

$$
\begin{gathered}
g_{\mu}=\frac{1}{|a| \sqrt{\mu_{+}^{2}+\mu_{-}^{2}}}, \\
m_{\mu}=\frac{\mu_{+}^{2}}{\mu_{+}^{2}+\mu_{-}^{2}},
\end{gathered}
$$

$\mathrm{cn}^{-1}\left(\cos \varphi \mid m_{\mu}\right)$ is the inverse Jacobian elliptic function, and $F\left(\varphi \mid m_{\mu}\right)$ is the incomplete elliptic integral of the first kind. Therefore, the integral $\mathscr{J}_{\mu}(\mu)$ obtained from the integration over any range of $\mu$ can be expressed as a combination of inverse Jocabian elliptic functions.

For the purpose of tracing photons from a distant observer back to the surface of a star located at $\left(r_{\star}, \theta_{\star}, \phi_{\star}\right)$, we integrate the trajectory of each photon from $\mu_{0}$ to $\mu_{\star}$. For those photons that can finally reach the star surface, some of them may reach the star surface when they first approach $\mu=\mu_{\star}$. For these photons, the $\mu$-integral $\mathscr{I}_{\mu, 1}$ can be obtained by

$$
\mathscr{I}_{\mu, 1}= \begin{cases}-\left[\mathscr{F}_{\mu}\left(\mu_{0}\right)-\mathscr{F}_{\mu}\left(\mu_{\star}\right)\right], & \text { if } \beta>0 \text { and } \mu_{0}<\mu_{\star}, \\ -\left[\mathscr{F}_{\mu}\left(\mu_{0}\right)+\mathscr{F}_{\mu}\left(\mu_{\star}\right)\right], & \text { if } \beta>0 \text { and } \mu_{0}>\mu_{\star}, \\ +\left[\mathscr{F}_{\mu}\left(\mu_{0}\right)-\mathscr{F}_{\mu}\left(\mu_{\star}\right)\right], & \text { if } \beta<0 \text { and } \mu_{0}>\mu_{\star}, \\ +\left[-2 \mathscr{F}_{\mu}(0)+\mathscr{F}_{\mu}\left(\mu_{0}\right)+\mathscr{F}_{\mu}\left(\mu_{\star}\right)\right], & \text { if } \beta<0 \text { and } \mu_{0}<\mu_{\star} .\end{cases}
$$

Some photons may not reach the star surface when they first reach $\mu=\mu_{\star}$. However, they may reach the star surface after their trajectory, turning back from $\mu_{+}$or $-\mu_{+}$to $\mu_{\star}$ again. For this case, we have

$$
\mathscr{I}_{\mu, 2}=\mathscr{I}_{\mu, 1}+\delta \mathscr{I}_{\mu, \text { even }}
$$

where

$$
\delta \mathscr{I}_{\mu, \text { even }}= \begin{cases}-2\left[\mathscr{F}_{\mu}(0)-\mathscr{F}_{\mu}\left(\mu_{\star}\right)\right], & \text { if } \beta>0 \text { and } \mu_{0}<\mu_{\star}, \\ -2\left[2 \mathscr{F}_{\mu}(0)-\mathscr{F}_{\mu}\left(\mu_{\star}\right)\right], & \text { if } \beta>0 \text { and } \mu_{0}>\mu_{\star}, \\ -2\left[2 \mathscr{F}_{\mu}(0)-\mathscr{F}_{\mu}\left(\mu_{\star}\right)\right], & \text { if } \beta<0 \text { and } \mu_{0}>\mu_{\star}, \\ -2\left[\mathscr{F}_{\mu}(0)-\mathscr{F}_{\mu}\left(\mu_{\star}\right)\right], & \text { if } \beta<0 \text { and } \mu_{0}<\mu_{\star} .\end{cases}
$$


Consequently, some photons may reach the star surface after their trajectories turn back to $\mu_{\star}$ for the third time. For this case, we have

$$
\mathscr{I}_{\mu, 3}=\mathscr{I}_{\mu, 2}+\delta \mathscr{I}_{\mu, \text { odd }},
$$

where

$$
\delta \mathscr{I}_{\mu, \text { odd }}= \begin{cases}-2\left[2 \mathscr{F}_{\mu}(0)-\mathscr{F}_{\mu}\left(\mu_{\star}\right)\right], & \text { if } \beta>0 \text { and } \mu_{0}<\mu_{\star}, \\ -2\left[\mathscr{F}_{\mu}(0)-\mathscr{F}_{\mu}\left(\mu_{\star}\right)\right], & \text { if } \beta>0 \text { and } \mu_{0}>\mu_{\star}, \\ -2\left[\mathscr{F}_{\mu}(0)-\mathscr{F}_{\mu}\left(\mu_{\star}\right)\right], & \text { if } \beta<0 \text { and } \mu_{0}>\mu_{\star}, \\ -2\left[2 \mathscr{F}_{\mu}(0)-\mathscr{F}_{\mu}\left(\mu_{\star}\right)\right], & \text { if } \beta<0 \text { and } \mu_{0}<\mu_{\star} .\end{cases}
$$

Therefore, if some photons reach the star surface when their trajectories reach $\mu_{\star}$ for the $2 k$-th time $[k(>1)$ is a integer], then we have

$$
\mathscr{I}_{\mu, 2 k}=\mathscr{I}_{\mu, 2 k-1}+\delta \mathscr{I}_{\mu, \mathrm{even}}
$$

while we have

$$
\mathscr{I}_{\mu, 2 k+1}=\mathscr{I}_{\mu, 2 k}+\delta \mathscr{I}_{\mu, \text { odd }},
$$

if some photons reach the star surface when their trajectories reach $\mu_{\star}$ for the $(2 k+1)$-th time. For most photons included in this paper, $k \leq 2-3$.

\section{A.2. Integral Over the r-direction}

We denote the integral over $r$ as

$$
\mathscr{F}_{r}(r)=\int \frac{d \tilde{r}}{\sqrt{R(\tilde{r})}}
$$

which can also be expressed in the form of the inverse Jacobian elliptic functions. To do so, we need to first solve equation $R(r)=0$ and find its four roots, denoted as $r_{1}, r_{2}, r_{3}$, and $r_{4}$, respectively. Since $R(r \rightarrow \pm \infty) \rightarrow \infty$ and $R(r=0)=-a^{2} q^{2}$, the solution of $R(r)=0$ can be divided into two cases: case A, $R(r)=0$ has two real roots and two complex roots; and case B, $R(r)=0$ has four real roots (e.g.,see Chandrasekhar 1983; Rauch \& Blandford 1994; Cadež et al. 1998; Yu \& Lu 2000; Lu \& Yu 2001; Li et al. 2005; Dexter \& Agol 2009). Below, we consider these two cases separately.

Case A: we assume that $r_{1}$ and $r_{2}$ represent the two complex roots, and $r_{3}$ and $r_{4}\left(r_{4}<r_{3}\right)$ represent the two real roots, respectively. Therefore, we have $r_{1}=r_{2}^{*}$, where $r_{2}^{*}$ represents the complex conjugate of $r_{2}$. Since $r_{1} r_{2} r_{3} r_{4}=$ $\left|r_{1}\right|^{2} r_{3} r_{4}=-a^{2} q^{2} \leq 0, r_{4}$ must be $\leq 0$ and $r_{3} \geq 0$, but $r_{3}$ cannot be equal to $r_{4}$. Since $R(r)$ must be $\geq 0$, we have either $r \geq r_{3}$ or $r \leq r_{4}$, and then the physically allowed region for $r$ must be $r \geq r_{3}$. We assume that the real part of the two complex roots $r_{1}$ and $r_{2}$ is $u$, while the imaginary parts of $r_{1}$ and $r_{2}$ are $w$ and $-w$, respectively. Therefore, the two complex roots can be expressed as $r_{1}=u+i w$ and $r_{2}=u-i w$, respectively. The two real roots can be expressed as $r_{3}=-u+v$ and $r_{4}=-u-v$, respectively, where $v$ is a real positive number. Now we can denote the function $\mathscr{F}_{r}(r)$ explicitly as

$$
\mathscr{F}_{r 2}(r)=\int_{r_{3}}^{r} \frac{d \tilde{r}}{\sqrt{R(\tilde{r})}}=\int_{r_{3}}^{r} \frac{d \tilde{r}}{\sqrt{\left(\tilde{r}-r_{1}\right)\left(\tilde{r}-r_{2}\right)\left(\tilde{r}-r_{3}\right)\left(\tilde{r}-r_{4}\right)}} .
$$

According to Byrd \& Friedman (1954, p135, eq. 260.00), the above function can be expressed in terms of an inverse Jacobian elliptic function, i.e.,

$$
\mathscr{F}_{r 2}(r)=g_{2} \mathrm{cn}^{-1}\left(\cos \varphi \mid m_{2}\right)=g_{2} F\left(\varphi \mid m_{2}\right),
$$

where

$$
\begin{gathered}
g_{2}=\frac{1}{\sqrt{A B}}, \\
m_{2}=\frac{(A+B)^{2}-4 v^{2}}{4 A B}, \\
A=\sqrt{(v-2 u)^{2}+w^{2}}, \\
B=\sqrt{(v+2 u)^{2}+w^{2}} \\
\varphi=\cos ^{-1}\left[\frac{(A-B) r+(u+v) A-(u-v) B}{(A+B) r+(u+v) A+(u-v) B}\right] .
\end{gathered}
$$

It is easy to verify here that $0<m_{2}<1$ and $\cos ^{2} \varphi \leq 1$. Therefore, the elliptical integral $F\left(\varphi \mid m_{2}\right)$ in Equation (A17) is well defined for $r \geq r_{3}$. Therefore, $\mathscr{J}_{r}$ may also be expressed as a combination of inverse Jacobian elliptic functions. 
In this case, some photons may reach the star surface with $r$ monotonically decreasing from $r_{0}$ to $r_{\text {hit }}$, and some other photons may reach the star surface with $r$ first decreasing from $r_{0}$ to $r_{3}$ and then turning back to $r_{\text {hit }}$. Hence, we should have

$$
\mathscr{J}_{r}=-\left[\mathscr{F}_{r 2}\left(r_{0}\right)-\mathscr{F}_{r 2}\left(r_{\text {hit }}\right)\right]
$$

and

$$
\mathscr{J}_{r}=-\left[\mathscr{F}_{r 2}\left(r_{0}\right)+\mathscr{F}_{r 2}\left(r_{\text {hit }}\right)\right]
$$

respectively.

Case B: assuming that $r_{1} \geq r_{2} \geq r_{3} \geq r_{4}$. Since $r_{1} r_{2} r_{3} r_{4}=-a^{2} q^{2} \leq 0$ and $r_{1}+r_{2}+r_{3}+r_{4}=0$, we have at least $r_{4} \leq 0$ and $r_{1}>0$. Therefore, the allowed physical regions for photons with $R(r) \geq 0$ are $r \geq r_{1}$ (region I) and $r_{3} \leq r \leq r_{2}$ (region II, if $r_{3}>0$ ). For those photons in region I, i.e., $r \geq r_{1}$, we denote the function $\mathscr{F}_{r}(r)$ as

$$
\mathscr{F}_{r 4, \mathrm{I}}(r)=\int_{r_{1}}^{r} \frac{d \tilde{r}}{\sqrt{R(\tilde{r})}}=\int_{r_{1}}^{r} \frac{d \tilde{r}}{\left(\tilde{r}-r_{1}\right)\left(\tilde{r}-r_{2}\right)\left(\tilde{r}-r_{3}\right)\left(\tilde{r}-r_{4}\right)} .
$$

According to Byrd \& Friedman (1954, p128, eq. 258.00), Equation (A25) can be expressed in terms of an inverse Jacobian elliptic function, i.e.,

$$
\mathscr{F}_{r 4, \mathrm{I}}(r)=g_{4} \mathrm{sn}^{-1}\left(\sin \varphi \mid m_{4}\right)=g_{4} F\left(\varphi \mid m_{4}\right),
$$

where

$$
\begin{gathered}
g_{4}=\frac{2}{\sqrt{\left(r_{1}-r_{3}\right)\left(r_{2}-r_{4}\right)}}, \\
\varphi=\sin ^{-1}\left(\sqrt{\frac{\left(r_{2}-r_{4}\right)\left(r-r_{1}\right)}{\left(r_{1}-r_{4}\right)\left(r-r_{2}\right)}}\right), \\
m_{4}=\frac{\left(r_{2}-r_{3}\right)\left(r_{1}-r_{4}\right)}{\left(r_{1}-r_{3}\right)\left(r_{2}-r_{4}\right)} .
\end{gathered}
$$

For this region, if $r_{1} \neq r_{2}, 0 \leq m_{4} \leq 1$ and thus Equation (A26) is well defined. If $r_{1}=r_{2}, m_{4}=1$ and $\sin ^{2} \varphi=1$, and thus Equation $(\mathrm{A26}) \propto \mathrm{sn}^{-1}(1 \mid 1)=\infty$, which is not well defined. If $r_{1}=r_{2}$, however, the integration in Equation (A25) can be directly obtained as

$$
\int_{r_{1}}^{r} \frac{d \tilde{r}}{\sqrt{R(\tilde{r})}}=\mathscr{H}_{r 4, \text { Ieq }}(r)=\frac{1}{\sqrt{\left(r_{1}-r_{3}\right)\left(r_{1}-r_{4}\right)}} \ln \left[\frac{\sqrt{\left(r-r_{3}\right)\left(r-r_{4}\right)}}{r-r_{1}}+\frac{r_{1}^{2}+r_{3} r_{4}+2 r_{1} r}{\left(r-r_{1}\right) \sqrt{\left(r_{1}-r_{3}\right)\left(r_{1}-r_{4}\right)}}\right] .
$$

For photons in the region I, some photons may reach the star surface with $r$ monotonically decreasing from $r_{0}$ to $r_{\text {hit }}$, while some other photons may reach the star surface with $r$ first monotonically decreasing from $r_{0}$ to $r_{1}$ and then turning back to the star surface. If $r_{1} \neq r_{2}$, hence, we should also have

$$
\mathscr{J}_{r}=-\left[\mathscr{F}_{r 4, \mathrm{I}}\left(r_{0}\right)-\mathscr{F}_{r 4, \mathrm{I}}\left(r_{\mathrm{hit}}\right)\right] \text {, }
$$

and

$$
\mathscr{J}_{r}=-\left[\mathscr{F}_{r 4, \mathrm{I}}\left(r_{0}\right)+\mathscr{F}_{r 4, \mathrm{I}}\left(r_{\text {hit }}\right)\right],
$$

respectively; if $r_{1}=r_{2}$ otherwise, we should have

$$
\mathscr{J}_{r}=-\left[\mathscr{H}_{r 4, \text { Ieq }}\left(r_{0}\right)-\mathscr{H}_{r 4, \text { Ieq }}\left(r_{\text {hit }}\right)\right] \text {, }
$$

and

$$
\mathscr{J}_{r}=-\left[\mathscr{H}_{r 4, \text { Ieq }}\left(r_{0}\right)+\mathscr{H}_{r 4 \text { Ieq }}\left(r_{\text {hit }}\right)\right]
$$

respectively.

For those photons in the region II, i.e., $r_{2} \geq r \geq r_{3}$, we denote $\mathscr{F}_{r}(r)$ as

$$
\mathscr{F}_{r 4, \mathrm{II}}(r)=\int_{r}^{r_{2}} \frac{d \tilde{r}}{\sqrt{R(\tilde{r})}}=\int_{r}^{r_{2}} \frac{d \tilde{r}}{\left(\tilde{r}-r_{1}\right)\left(\tilde{r}-r_{2}\right)\left(\tilde{r}-r_{3}\right)\left(\tilde{r}-r_{4}\right)} .
$$

According to Byrd \& Friedman (1954, p116, eq. 255.00), Equation (A35) can be expressed in terms of an inverse Jacobian elliptic function, i.e.,

$$
\mathscr{F}_{r 4, \mathrm{II}}(r)=g_{4} \mathrm{sn}^{-1}\left(\sin \varphi \mid m_{4}\right)=g_{4} F\left(\varphi \mid m_{4}\right),
$$

where

$$
\varphi=\sin ^{-1}\left(\sqrt{\frac{\left(r_{1}-r_{3}\right)\left(r_{2}-r\right)}{\left(r_{2}-r_{3}\right)\left(r_{1}-r\right)}}\right),
$$


$g_{4}$, and $m_{4}$ are the same as that given by Equations (A27) and A29, respectively. Similar to Equation (A26), Equation A36 is well defined if $r_{1} \neq r_{2}$ as $0 \leq m_{4} \leq 1$ and $\sin ^{2} \varphi<1$. If $r_{1}=r_{2}$, then Equation (A36) can be directly replaced by

$$
\int_{r}^{r_{1}} \frac{d \tilde{r}}{\sqrt{R(\tilde{r})}}=\mathscr{H}_{r 4 \text { IIeq }}(r)=\frac{1}{\sqrt{\left(r_{1}-r_{3}\right)\left(r_{1}-r_{4}\right)}} \ln \left[\frac{\sqrt{\left(r-r_{3}\right)\left(r-r_{4}\right)}}{r_{1}-r}+\frac{r_{1}^{2}+r_{3} r_{4}+2 r_{1} r}{\left(r_{1}-r\right) \sqrt{\left(r_{1}-r_{3}\right)\left(r_{1}-r_{4}\right)}}\right] .
$$

For photons in the region II, some of them may reach the star surface with $r$ monotonically decreasing from $r_{0}$ to $r_{\text {hit }}$, while some others may reach the star surface with $r$ first monotonically decreasing from $r_{0}$ to $r_{3}$ and then turning back to the star surface. For these two cases, if $r_{0} \leq r_{2}$ and $r_{2} \neq r_{3}$, we should have

$$
\mathscr{J}_{r}=\left[\mathscr{F}_{r 4, \mathrm{II}}\left(r_{0}\right)-\mathscr{F}_{r 4, \mathrm{II}}\left(r_{\text {hit }}\right)\right] \text {, }
$$

and

$$
\mathscr{J}_{r}=-2 \mathscr{F}_{r 4, \mathrm{II}}\left(r_{3}\right)+\left[\mathscr{F}_{r 4, \mathrm{II}}\left(r_{0}\right)+\mathscr{F}_{r 4, \mathrm{II}}\left(r_{\text {hit }}\right)\right]
$$

respectively; if $r_{0} \leq r_{1}$ and $r_{1}=r_{2}$ otherwise, we should have

$$
\mathscr{J}_{r}=\left[\mathscr{H}_{r 4, \text { IIeq }}\left(r_{0}\right)-\mathscr{H}_{r 4, \text { IIeq }}\left(r_{\text {hit }}\right)\right],
$$

and

$$
\mathscr{J}_{r}=-2 \mathscr{H}_{\mathrm{r} 4, \mathrm{II}}\left(r_{3}\right)+\left[\mathscr{H}_{r 4, \mathrm{II} \text { eq }}\left(r_{0}\right)+\mathscr{H}_{r 4, \mathrm{IIeq}}\left(r_{\text {hit }}\right)\right]
$$

respectively.

For the problem considered in this paper, the photons that we are interested in must be transported from the stars rotating around the central $\mathrm{MBH}$ to a distant observer. Our calculations show that their trajectories should not be bended too much to oscillate within the region II (i.e., $r_{3} \leq r \leq r_{2}$ ) for many times.

\section{A.3. $r_{\text {hit }}$}

According to Equation (A1), $\mathscr{I}_{\mu}=\mathscr{J}_{r}$, so $r_{\text {hit }}$ can be derived from this equation once the two integrals are obtained as described above. If a photon reaches $\mu=\mu_{\star}$ for $k$ times, then $\mathscr{I}_{\mu}=\mathscr{I}_{\mu, k}$ (eqs. A15 and A16).

Case A of the $r$-integral:

1. If a photon reaches the star surface before it encounters the turning point $r_{3}$, then

$$
\mathscr{I}_{\mu, k}=-\left[\mathscr{F}_{r 2}\left(r_{0}\right)-\mathscr{F}_{r 2}\left(r_{\mathrm{hit}, k}\right)\right]
$$

Therefore, we have the solution

$$
r_{\mathrm{hit}, k}=\mathscr{F}_{r 2}^{-1}\left(\mathscr{I}_{\mu, k}+\mathscr{F}_{r 2}\left(r_{0}\right)\right) .
$$

We define $s_{21}=\mathscr{I}_{\mu, k}+\mathscr{F}_{r 2}\left(r_{0}\right)$, then we may express $r_{\mathrm{hit}, k}$ as

$$
r_{\text {hit }, k}=\mathscr{F}_{r 2}^{-1}(s)=\frac{b_{1}-b_{2} \cos \varphi}{a_{2} \cos \varphi-a_{1}}
$$

where

$$
\begin{gathered}
a_{1}=A-B, \\
a_{2}=A+B, \\
b_{1}=-(u+v) A+(u-v) B, \\
b_{2}=-(u+v) A-(u-v) B,
\end{gathered}
$$

and

$$
\cos \varphi=\operatorname{cn}\left(\frac{s_{21}}{g_{2}} \mid m_{2}\right)
$$

and $m_{2}$ is given by Equation (A19).

2. If a photon reaches the star surface after it encounters the turning point $r_{3}$, according to Equation (A24), then we have

$$
r_{\text {hit }, k}=\mathscr{F}_{r 2}^{-1}\left(s_{22}\right)=\frac{b_{1}-b_{2} \cos \varphi}{a_{2} \cos \varphi-a_{1}}
$$

where

$$
s_{22}=-\mathscr{I}_{\mu, k}-\mathscr{F}_{r 2}\left(r_{0}\right),
$$

and $a_{1}, a_{2}, b_{1}, b_{2}$, and $\cos \varphi$ are given by Equations (A46)-A - If $s_{22}>\mathscr{F}_{r 2}(\infty)$, then there is no solution for $r_{\text {hit }, k}$, and therefore it is not necessary to go to larger $k$ to search for the solution of $r_{\text {hit, }, k}$. 
Case B of the $r$-integral:

1. For photons in the allowed region $r \geq r_{1}$ (but $r_{1} \neq r_{2}$ ), if they reach the star surface before they encounter the turning point $r_{1}$, according to Equation (A31) we should have

$$
r_{\mathrm{hit}, k}=\mathscr{F}_{r 4, \mathrm{I}}^{-1}\left(s_{41}\right)=\frac{r_{2} C-r_{1}}{C-1},
$$

where

$$
\begin{gathered}
s_{41}=\mathscr{I}_{\mu, k}+\mathscr{F}_{r 4, \mathrm{I}}\left(r_{0}\right), \\
C=\frac{r_{1}-r_{4}}{r_{2}-r_{4}} \sin ^{2} \varphi, \\
\sin \varphi=\operatorname{sn}\left(\frac{s_{41}}{g_{4}} \mid m_{4}\right),
\end{gathered}
$$

$g_{4}$ and $m_{4}$ are the same as that given by Equations (A27) and (A29), respectively.

2. For photons in the allowed region $r \geq r_{1}$ (but $r_{1} \neq r_{2}$ ), if they reach the star surface after they encounter the turning point $r_{1}$, according to Equation (A32) we should have

$$
r_{\text {hit }, k}=\mathscr{F}_{r 4, \mathrm{I}}^{-1}\left(s_{42}\right)=\frac{r_{2} C-r_{1}}{C-1},
$$

where

$$
\begin{gathered}
s_{42}=-\mathscr{I}_{\mu, k}-\mathscr{F}_{r 4, \mathrm{I}}\left(r_{0}\right), \\
\sin \varphi=\operatorname{sn}\left(\frac{s_{42}}{g_{4}} \mid m_{4}\right),
\end{gathered}
$$

$C, g_{4}$, and $m_{4}$ are the same as that given by Equations (A56), (A27) and (A29), respectively. If $s_{42}>-\mathscr{I}_{\mu, k}-$ $\mathscr{F}_{r 4, \mathrm{I}}(\infty)$, then there is no solution for $r_{\mathrm{hit}, k}$, and it is not necessary to go to larger $k$ to search for the solution of $r_{\text {hik }, k}$.

3. For photons in the allowed region $r \geq r_{1}$ and $r_{1}=r_{2}$, if the reach the star surface before they encounter the turning point $r_{1}$, according to Equation (A33) we should have

$$
r_{\mathrm{hit}, k}=\mathscr{H}_{r 4, \text { Ieq }}^{-1}\left(s_{43}\right),
$$

where

$$
s_{43}=\mathscr{I}_{\mu, k}+\mathscr{H}_{r 4, \text { Ieq }}\left(r_{0}\right) .
$$

4. For photons in the allowed region $r \geq r_{1}$ and $r_{1}=r_{2}$, if they reach the star surface after they encounter the turning point $r_{1}$, according to Equation (A33) we should have

$$
r_{\text {hit }, k}=\mathscr{H}_{r 4, \text { Ieq }}^{-1}\left(s_{44}\right),
$$

where

$$
s_{44}=-\mathscr{I}_{\mu, k}-\mathscr{H}_{r, \mathrm{Ieq}}\left(r_{0}\right) .
$$

If $s_{44}>-\mathscr{I}_{\mu, k}-\mathscr{H}_{r 4, \mathrm{II}}(\infty)$, then there is no solution for $r_{\mathrm{hit}, k}$, and consequently it is not necessary to go to larger $k$ to search for the solution of $r_{\text {hit }, k}$.

5. For photons in the allowed region $r_{2} \geq r \geq r_{3}$, they cannot transport to the distant observer if $r_{0} \gg r_{2}$, and therefore are not interested in this study.

\section{A.4. Longitude and Time}

The motion of a photon in the $\phi$ - and $t$-directions can be also obtained as (e.g., see Rauch \& Blandford 1994)

$$
\phi=r_{\text {sign }} \int^{r} \frac{\lambda r^{2}+2 r(a-\lambda)}{r^{2}-2 r+a^{2}} \frac{d r}{\sqrt{R(r)}}+\theta_{\text {sign }} \int^{\mu} \frac{\lambda \mu^{2}}{1-\mu^{2}} \frac{d \mu}{\sqrt{\Theta_{\mu}}}
$$

and

$$
t=r_{\mathrm{sign}} \int^{r} \frac{r^{4}+a^{2} r^{2}+2 a r(a-\lambda)}{r^{2}-2 r+a^{2}} \frac{d r}{\sqrt{R(r)}}+\theta_{\mathrm{sign}} \int^{\mu} a^{2} \mu^{2} \frac{d \mu}{\sqrt{\Theta_{\mu}}}
$$


provided that the motion of the photon in the $r$ - and $\theta$-directions are given (see Sections A.1 and A.2). In Equations (A64) and (A65), $r_{\text {sign }}$ and $\theta_{\text {sign }}$ are the signs of the $r$ - and $\theta$-integrals, which can be obtain by solving Equation (A1) (see details in Sections A.2 and A.1). The integrations on the right sides of Equations (A64) and (A65) over either $r$ or $\theta$ can be expanded similar to those done in Sections A.2 and A.1 according to $r_{\text {sion }}$ and $\theta_{\text {sign }}$ and how many times that they change sign; we do not show it here in detail. The $r$-terms in Equations (A64) and (A65) can be accurately integrated by adopting the Gauss-Kronrod integration scheme (see also Rauch \& Blandford 1994). The $\theta$-terms can be obtained analytically by the elliptical functions as

$$
\begin{aligned}
\mathscr{K}_{\mu}(\mu) & \equiv \int_{\mu}^{\mu_{+}} \frac{\tilde{\mu}^{2}}{1-\tilde{\mu}^{2}} \frac{d \tilde{\mu}}{\sqrt{\Theta_{\mu}(\tilde{\mu})}}=\int_{\mu}^{\mu_{+}} \frac{1}{1-\tilde{\mu}^{2}} \frac{d \tilde{\mu}}{\sqrt{\Theta_{\mu}(\tilde{\mu})}}-\int_{\mu}^{\mu_{+}} \frac{d \tilde{\mu}}{\sqrt{\Theta_{\mu}(\tilde{\mu})}} \\
& =\frac{1}{1-\mu_{+}^{2}} g_{\mu} \Pi\left(\cos ^{-1} \frac{\mu}{\mu_{+}} \mid n_{\mu}, m_{\mu}\right)-g_{\mu} \mathscr{F}\left(\cos ^{-1} \frac{\mu}{\mu_{+}} \mid m_{\mu}\right),
\end{aligned}
$$

according to Byrd \& Friedman (1954, p48, eq. 213.02), and

$$
\begin{aligned}
\mathscr{L}_{\mu}(\mu) & \equiv \int_{\mu}^{\mu_{+}} \tilde{\mu}^{2} \frac{d \tilde{\mu}}{\sqrt{\Theta_{\mu}(\tilde{\mu})}}=\frac{1}{|a|} \int_{\mu}^{\mu_{+}} \sqrt{\frac{\mu_{-}^{2}+\tilde{\mu}^{2}}{\mu_{+}^{2}-\tilde{\mu}^{2}}} d \tilde{\mu}-\mu_{-}^{2} \int_{\mu}^{\mu_{+}} \frac{d \tilde{\mu}}{\sqrt{\Theta_{\mu}(\tilde{\mu})}} \\
& =\left(\mu_{+}^{2}+\mu_{-}^{2}\right) g_{\mu} \mathscr{E}\left(\cos ^{-1} \frac{\mu}{\mu_{+}} \mid m_{\mu}\right)-\mu_{-}^{2} g_{\mu} \mathscr{F}\left(\cos ^{-1} \frac{\mu}{\mu_{+}} \mid m_{\mu}\right),
\end{aligned}
$$

according to Byrd \& Friedman (1954, p48, eq. 213.01), where $\mathscr{F}, \mathscr{E}$, and $\Pi$ are the elliptical integral of the first, second, and third kind, respectively, $g_{\mu}$ and $m_{\mu}$ are given by Equations (A8) and (A9), and $n_{\mu}$ is given by

$$
n_{\mu}=\frac{\mu_{+}^{2}}{1-\mu_{+}^{2}} .
$$

\section{A.5. Apparent Position of a Star Close to the GC MBH in the Observer's Sky Plane}

It is necessary to develop a fast and accurate method to find those rays propagating from the observer to the star. The most simple and direct method is to perform ray tracing calculations for a large number of rays with various $(\alpha$, $\beta$ ) and search for the minimum of the distance function $d(\alpha, \beta)=d\left(r_{\text {hit }}, \theta_{\star}, \phi_{\text {hit }}\right)$ (defined by Eq. 27). However, this method may be not efficient in finding the ray that actually hits on the star at any given position because (1) not every ray with arbitrary $(\alpha, \beta)$ can end up at the surface of the cone with $\theta=\theta_{\star}$; and $(2)$ the contours for $d(\alpha, \beta)$ appear to be extremely narrow elliptically in some cases; thus, the convergence for numerically finding the local minimum of $d$ is slow.

In this study, we implement a new method to find the local minimum of $d(\alpha, \beta)$. We assume a flat space with coordinate $\left(r^{\prime}, \theta^{\prime}, \phi^{\prime}\right)$ as an approximation to the Kerr space. In this flat space, those rays from the distant observer that can hit the position $\left(r^{\prime}, \theta_{\star}, \phi^{\prime}\right)$ have

$$
\alpha=r^{\prime} \sin \theta_{\star} \sin \phi^{\prime}, \quad \beta=r^{\prime} \cos \theta_{\star} \sin \theta_{\mathrm{o}}-r^{\prime} \sin \theta_{\star} \cos \phi^{\prime} \cos \theta_{\mathrm{o}} .
$$

Here and hereafter the superscript ' indicates that a quantity is defined in the flat space. Since the example stars are still far away from the event horizon, the trajectories of photons emitted from those stars do not deviate away from the corresponding cases in a flat space too much. Therefore, the local minimum of the distance function $d$ can be found by the following procedures.

1. We first assume $\left(r_{0}^{\prime}, \phi_{0}^{\prime}\right)=\left(r_{\star}, \phi_{\star}\right)$ to obtain $\left(\alpha_{0}, \beta_{0}\right)$, which is taken as the guess position of the star $\left[\left(r_{\star}, \theta_{\star}, \phi_{\star}\right)\right.$ in the Kerr space] on the observer's sky plane. Adopting $(\alpha, \beta)=\left(\alpha_{0}, \beta_{0}\right)$, we perform ray tracing calculations in the Kerr metric to find the position of the ray hitting the surface $\theta=\theta_{\star}$, i.e., $\left(r_{0}, \theta_{\star}, \phi_{0}\right)$, which may deviate from the real position of the star $\left(r_{\star}, \theta_{\star}, \phi_{\star}\right)$ because of the curved spacetime. We calculate the distance between $\left(r_{\star}, \theta_{\star}, \phi_{\star}\right)$ and $\left(r_{0}, \theta_{\star}, \phi_{0}\right)$ as $d_{0}=d\left(\alpha_{0}, \beta_{0}\right)$.

2. We select a number of points (e.g., eight points) on a circle with radius of $d_{0}^{\prime}=d_{0}$ around $\left(r_{0}^{\prime}, \theta_{\star}, \phi_{0}^{\prime}\right)$. For the $j$-th point $(j=1, \ldots, 8)$ selected on the circle, i.e., $\left(r_{1, j}^{\prime}, \theta_{*}, \phi_{1, j}^{\prime}\right)$, we can obtain $\left(\alpha_{1, j}, \beta_{1, j}\right)$ according to Equation (A69). Using $\left(\alpha_{1, j}, \beta_{1, j}\right)$, we perform ray tracing calculations again and find the position $\left(r_{1, j}, \theta_{\star}, \phi_{1, j}\right)$ that the ray hits on the surface $\theta=\theta_{\star}$, and consequently, we obtain the distance of that position from the star as $d_{1, j}=d\left(\alpha_{1, j}, \beta_{1, j}\right)$. Among those (eight) points on the circle, we find the one [denoted by $\left(r_{1, \mathrm{~m}}, \theta_{\star}, \phi_{1, \mathrm{~m}}\right)$ ] with the smallest distance $d$ (denoted by $d_{1, \mathrm{~m}}$ ), and the corresponding point in the flat space is denoted by $\left(r_{1, \mathrm{~m}}^{\prime}, \theta_{\star}, \phi_{1, \mathrm{~m}}^{\prime}\right)$.

3. If $d_{1, \mathrm{~m}} \leq \xi r_{\star}\left(\xi=10^{-6} \sim 10^{-8}\right)$, then we find the apparent position of the star and the calculation is terminated; if $d_{1, \mathrm{~m}}>\xi r_{\star}$ and $d_{1, \mathrm{~m}}>d_{0}$, then we adopt the same point $\left(r_{0}^{\prime}, \theta_{*}, \phi_{0}^{\prime}\right)$ in the flat space as the center of a new circle with a radius of $d_{1}^{\prime}=d_{0}^{\prime} / 5$; otherwise, we choose the point $\left(r_{1, \mathrm{~m}}^{\prime}, \theta_{*}, \phi_{1, \mathrm{~m}}^{\prime}\right)$ in the flat space as the center of the new circle and its radius is set to be $d_{1}^{\prime}=d_{0}^{\prime}$. 
4. We select a number of points $\left(r_{2, j}^{\prime}, \theta_{\star}, \phi_{2, j}^{\prime}\right)$ on the new circle and obtain $\left(\alpha_{2, j}, \beta_{2, j}\right)$. We further perform ray tracing calculations and find the position $\left(r_{2, j}, \theta_{\star}, \phi_{2, j}\right)$ that the ray hits on the surface $\theta=\theta_{\star}$. We calculate the distance of that position from the star $\left(r_{\star}, \theta_{\star}, \phi_{\star}\right)$, i.e., $d_{2, j}=d\left(\alpha_{2, j}, \beta_{2, j}\right)$ in the Kerr space. Among those selected points, we find the one with the smallest $d$, i.e., $d_{2, \mathrm{~m}}$, and the corresponding point in the flat space is denoted as $\left(r_{2, \mathrm{~m}}^{\prime}, \theta_{*}, \phi_{2, \mathrm{~m}}^{\prime}\right)$.

5. Similar to the above procedures 3 and 4 , we do iterations until we find the apparent position of the star at $\left(r_{\star}, \theta_{\star}, \phi_{\star}\right)$ in the observer's sky plane.

The above method is fast in searching for the apparent position $(\alpha, \beta)$ of a star at $\left(r_{\star}, \theta_{\star}, \phi_{\star}\right)$ in the Kerr spacetime on the distant observer's sky plane, which enables an efficient MCMC fitting to the mock data obtained for those example stars.

\section{B. ANALYTICAL ESTIMATION OF THE SHIFT OF STAR APPARENT POSITIONS DUE TO THE LENSE-THIRRING PRECESSION AND THE FRAME DRAGGING}

The Lense-Thirring effect causes the precession of the orbital plane via the change of the longitude of the ascending node at a mean rate of

$$
<\dot{\Omega}>=\frac{2 a M_{\bullet}}{a_{\text {orb }}^{3}\left(1-e_{\text {orb }}^{2}\right)^{3 / 2}},
$$

and the change of the argument of periapsis at a mean rate

$$
<\dot{\Upsilon}>=-3 \dot{\Omega} \cos \zeta
$$

where $\Omega$ and $\Upsilon$ are the longitude of the ascending node and the argument of periapsis defined for the stellar orbital plane with the equatorial plane of the $\mathrm{BH}$ as the reference plane, and $\zeta$ is the angle between the orbital angular momentum of the star and the spin direction and given by $\zeta=\arccos \left(\cos I^{\prime} \cos i+\sin I^{\prime} \sin i \sin \left(\Omega^{\prime}-\epsilon\right)\right)$. The accumulated changes of $\Omega$ and $\Upsilon$ per orbit are

$$
\delta \Omega=2 \pi \frac{2 a r_{\mathrm{g}}^{3 / 2}}{a_{\mathrm{orb}}^{3 / 2}\left(1-e_{\mathrm{orb}}^{2}\right)^{3 / 2}},
$$

and

$$
\delta \Upsilon=-2 \pi \frac{6 a r_{\mathrm{g}}^{3 / 2}}{a_{\mathrm{orb}}^{3 / 2}\left(1-e_{\mathrm{orb}}^{2}\right)^{3 / 2}} \cos \zeta .
$$

Note that most of the changes in $\Omega$ and $\Upsilon$ take place near periapsis. The values of the changes are $\delta \Omega=0.0017^{\circ}$, $0.00059^{\circ}, 0.010^{\circ}, 0.14^{\circ}, 0.074^{\circ}, 0.0092^{\circ}$, and $\delta \Upsilon=0.0044^{\circ}, 0.0010^{\circ},-0.015^{\circ},-0.21^{\circ},-0.11^{\circ}$, and $-0.014^{\circ}$ for $\mathrm{S} 0-$ 2/S2, S0-102, Ea, Eb, Ec, and Ed, respectively. Considering the projection effect, the changes of $\Omega^{\prime}, \Upsilon^{\prime}$, and $I^{\prime}$ can be obtained by

$$
\begin{gathered}
\delta \Omega^{\prime}=\left[\cos i-\frac{\cos I^{\prime} \sin i \sin \left(\Omega^{\prime}-\epsilon\right)}{\sin I^{\prime}}\right] \delta \Omega, \\
\delta \Upsilon^{\prime}=-\frac{\sin i \sin \left(\Omega^{\prime}-\epsilon\right)}{\sin I^{\prime}} \delta \Omega+\delta \Upsilon,
\end{gathered}
$$

and

$$
\delta I^{\prime}=\sin i \cos \left(\Omega^{\prime}-\epsilon\right) \delta \Omega .
$$

If $\zeta \rightarrow 0^{\circ}$, i.e., the star orbit is close to being on the MBH equatorial plane, $I^{\prime} \rightarrow i, \Omega^{\prime}-\epsilon \rightarrow \pi / 2$, then $\delta \Omega^{\prime} \rightarrow 0$, $\delta \Upsilon^{\prime} \rightarrow-2 \delta \Omega$, and $\delta I^{\prime} \rightarrow 0$.

After the motion of one full orbit, the difference between the position of an example star in the sky plane at apoapsis/periapsis for the case with a rapidly spinning $\mathrm{BH}(a)$ and that with a non-spinning $\mathrm{BH}(a=0)$ is

$$
\begin{aligned}
\delta_{\mathrm{apo} / \mathrm{peri}} \simeq & a_{\mathrm{orb}}\left(1 \pm e_{\mathrm{orb}}\right)\left[\delta^{2} \Omega^{\prime}\left(1-\sin ^{2} \Upsilon^{\prime} \sin ^{2} I^{\prime}\right)+\delta^{2} \Upsilon^{\prime}\left(1-\cos ^{2} \Upsilon^{\prime} \sin ^{2} I^{\prime}\right)+\sin \Upsilon^{\prime 2} \sin ^{2} I^{\prime} \delta^{2} I^{\prime}\right. \\
& \left.+2 \cos I^{\prime} \delta \Omega^{\prime} \delta \Upsilon^{\prime}-2 \sin \Upsilon^{\prime} \cos \Upsilon^{\prime} \sin I^{\prime} \cos I^{\prime} \delta \Upsilon^{\prime} \delta I^{\prime}-2 \sin \Upsilon^{\prime} \cos \Upsilon^{\prime} \sin I^{\prime} \delta \Omega^{\prime} \delta I^{\prime}\right]^{1 / 2},
\end{aligned}
$$

where the signs ' + ' and '-' are for the difference at apoapsis $\left(\delta_{\text {apo }}\right)$ and periapsis $\left(\delta_{\text {peri }}\right)$, respectively.

We may also include the high-order precession due to the quadruple moment of the MBH by replacing $\delta \Omega$ and $\delta \Upsilon$ in Equations (B3) and (B34) by $\delta \Omega+\delta \Omega_{\mathrm{Q}}$ and $\delta \Upsilon+\delta \Upsilon_{\mathrm{Q}}$, respectively, where

$$
\delta \Omega_{\mathrm{Q}}=-\cos \zeta \frac{3 \pi a^{2} r_{\mathrm{g}}^{2}}{a_{\mathrm{orb}}^{2}\left(1-e_{\mathrm{orb}}^{2}\right)^{2}}
$$

and

$$
\delta \Upsilon_{\mathrm{Q}}=\frac{1-5 \cos ^{2} \zeta}{2} \frac{3 \pi a^{2} r_{\mathrm{g}}^{2}}{a_{\mathrm{orb}}^{2}\left(1-e_{\mathrm{orb}}^{2}\right)^{2}}
$$


See detailed derivations from Barker \& O'Connell (1975) and Wex \& Kopeikin (1999). 